\title{
COST-EFFECTIVENESS OF EARLY VERSUS DELAYED FUNCTIONAL RESTORATION FOR CHRONIC DISABLING OCCUPATIONAL MUSCULOSKELETAL DISORDERS
}

by

\author{
BRIAN ROHAN THEODORE \\ Presented to the Faculty of the Graduate School of \\ The University of Texas at Arlington in Partial Fulfillment \\ of the Requirements \\ for the Degree of
}

DOCTOR OF PHILOSOPHY

THE UNIVERSITY OF TEXAS AT ARLINGTON

May 2009 
Copyright (C) by Brian Rohan Theodore 2009

All Rights Reserved 


\section{ACKNOWLEDGEMENTS}

I would like to begin by thanking my Faculty Mentor and Dissertation Committee Chairman, Robert J. Gatchel, Ph.D., ABPP, for his expert guidance and mentoring in conceptualizing this project and facilitating its successful completion. Dr. Gatchel has also been an instrumental source of inspiration and support in my general academic career and has provided ample opportunities for me to grow as a researcher. Special thanks are also extended to my Committee Members, Tom G. Mayer, M.D., Perry N. Fuchs, Ph.D., Andrew Baum, Ph.D., and Angela L. Dougall, Ph.D., for their invaluable feedback and support in making this project a success.

This project was also a success due to the expertise of the management and staff at the Productive Rehabilitation Institute of Dallas for Ergonomics (PRIDE). I would like to especially thank Tom G. Mayer, M.D., PRIDE Medical Director, for his clinical insight and expertise in the rehabilitation of patients within the Workers' Compensation system. Special thanks are also extended to Keith Davis and Lisa Watten for providing seamless integration among the medical, billing, and research databases that were instrumental for this project. In addition, I would like to record my gratitude to Peter B. Polatin, M.D., for providing early guidance and reference material on the economic aspects of this project.

I would also like to thank Arnold Haas at the Ohio Bureau of Workers' Compensation for his time and effort in providing high-quality data on medical costs 
within the Workers' Compensation system. Special thanks are also due to Amy Lee and Lorena Lopez-Gonzales at the Texas Department of Insurance Workers' Compensation Division, for their time and effort in providing normative data and information on Texas State Workers' Compensation regulations. I am also tremendously grateful to Robbie Haggard, of the Eugene McDermott Center for Pain Management at the University of Texas Southwestern Medical Center, for providing access to normative quality-of-life and outcome data from a chronic pain population.

And last but not least, I would like to express a deep sense of gratitude for my family and friends. My dearest parents have been instrumental in providing emotional, moral, and financial support throughout my academic career. And my dearest family and friends have always been there at every step of the way, providing gems of wisdom, plenty of humor, and a sense of belonging.

March 13, 2009 


\begin{abstract}
COST-EFFECTIVENESS OF EARLY VERSUS DELAYED FUNCTIONAL RESTORATION FOR CHRONIC DISABLING OCCUPATIONAL MUSCULOSKELETAL DISORDERS
\end{abstract}

Brian Rohan Theodore, PhD.

The University of Texas at Arlington, 2009

Supervising Professor: Robert J. Gatchel

Chronic pain is among the most costly disorders in the United States. Societal impacts include productivity losses, disability compensation, and reduction in psychosocial well-being. To date, there is little documentation on the effect of extended disability on both treatment- and cost-effectiveness. The present study investigated the treatment- and cost-effectiveness of early versus delayed functional restoration for three groups, in a matched cohort design based on length of disability: Early Rehabilitation (48 months); Intermediate Duration (9-18 months); and Delayed Rehabilitation ( $>18$ months). Psychosocial deficits at pre-rehabilitation were significantly greater for the late rehabilitation groups, with approximately 2 times greater likelihood of Major Depressive Disorder and Opioid Dependence Disorder. At post-rehabilitation, all groups fared 
equally well on psychosocial measures, with no significant differences observed on oneyear objective, socioeconomic outcomes (e.g., $\geq 85 \%$ RTW regardless of disability duration). Economic analyses indicated that early rehabilitation was a cost-effective approach to managing chronic disability, despite comparable outcomes across the disability duration groups. Costs during the timeframe of the rehabilitation program were significantly and positively associated with Major Depressive Disorder and prior surgeries. When accounting for the costs accrued over the duration of disability, early rehabilitation yields an estimated savings in costs up to $66 \%$, or approximately $\$ 138,000$ per patient. The findings in this study are consistent with the biopsychosocial paradigm for the etiology of disability, and are also supported by previous documentation on the efficacy and cost-effectiveness of treatment modalities incorporating the biopsychosocial model of managing pain and disability. 


\section{TABLE OF CONTENTS}

ACKNOWLEDGEMENTS ........................................... iii

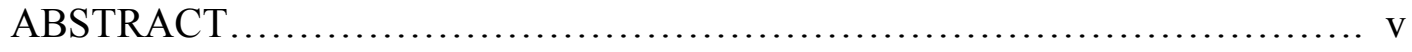

Chapter Page

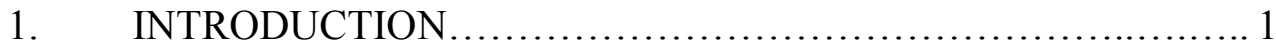

Theories of Pain............................................. 5

Development of Pain: From Acute to Chronic................... 9

Biopsychosocial Rehabilitation............................. 11

Application of Economic Theories in Healthcare................... 16

Costs and Outcomes in Economic Evaluations.................... 22

Analytical Methods for Economic Evaluations....................27

Cost-Effectiveness of Biopsychosocial Pain Interventions........ 35

Early versus Delayed Rehabilitation...............................39

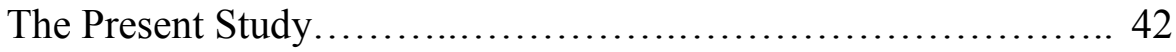

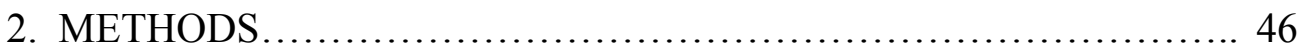

Patients.................................................... 46

Cohort Structure............................................48

Measures................................................... 51

Cost Estimation................................................ 53

Procedure..................................................... 61

vii 
3. RESULTS ................................................... 74

Demographic and Pre-Rehabilitation Characteristics................ 74

Treatment Efficacy of the Functional Restoration

Program.................................................. 80

Cost-Effectiveness Analysis of the Functional Restoration

Program............................................. 81

Cost Analysis on the Total Cost of Illness........................ 90

4. DISCUSSION .................................................. 99

Pre-Rehabilitation Characteristics.......................... 100

Treatment Efficacy....................................... 104

Cost-Effectiveness Analysis.............................. 107

Total Cost of Illness........................................ 110

General Discussion........................................ 112

Limitations of the Present Study............................. 115

Conclusion.............................................. 120

\section{APPENDIX}

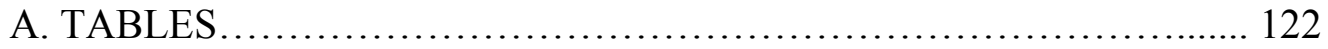

B. FIGURES ................................................. 174

C. COMPUTATION OF TEMPORARY INCOME

BENEFITS ..................................................... 185

D. COMPUTATION OF IMPAIRMENT INCOME

BENEFITS ................................................... 188

E. COMPUTATION OF SUPPLEMENTALINCOME BENEFITS. 
F. DATA FROM THE EUGENE MCDERMOTT CENTER

FOR PAIN MANAGEMENT .................................. 194

REFERENCES .............................................................. 196

BIOGRAPHICAL INFORMATION ...................................... 223 


\section{CHAPTER 1}

\section{INTRODUCTION}

Pain, and its resulting disability, is one of the most debilitating and costly disorders in the United States as well as in most industrialized countries. A recent study reported a population-based estimate of pain prevalence at approximately $27 \%$, at time of sampling, in the United States (Krueger \& Stone, 2008). In terms of healthcare utilization, pain accounts for more than $80 \%$ of all visits to healthcare providers (Gatchel \& Turk, 1996; Kerns, Otis, Rosenberg, \& Reid, 2003). The financial costs associated with pain are also substantial in magnitude. For example, the financial impact of low back pain alone is estimated at being in excess of $\$ 90$ billion in healthcare costs annually (Luo, Pietrobon, Sun, Liu, \& Hey, 2004). Annual medication costs associated with both prescription and non-prescription analgesics were estimated at $\$ 13.8$ billion and $\$ 2.6$ billion, respectively (Krueger $\&$ Stone, 2008). As noted by Gatchel and Okifuji (2006), as well as Stewart, Ricci, Chee, Morganstein, and Lipton (2003), after taking into account the costs of lost productivity in the labor workforce, the financial impact of pain is staggering.

A unique sub-population of pain patients correspond to occupational-related injuries and its consequent pain and disability. In the United States, most patients with occupational injuries fall under the jurisdiction of some variety of State or Federal Workers' Compensation systems. While the vast majority of injured workers recover 
and resume productivity, a small but significant percentage of these patients remain disabled in a chronic state (Gatchel \& Mayer, 2000; Mayer et al., 2003). Significantly, the disability compensation system has been identified as being associated with barriers to recovery among patients, relative to patients not receiving disability compensation (Flynn \& Hoque, 1979; Sander \& Meyers, 1986; Waddell et al., 1979). Reviews of State Workers' Compensation databases have indicated that as little as $7 \%$ of these chronically disabled patients account for about approximately $70 \%$ of the annual costs to the Workers' Compensation system (Hashemi, Webster, \& Clancy, 1998; Hashemi, Webster, Clancy, \& Volinn, 1997; Hashemi, Webster, Clancy, \& Courtney, 1998). Additionally, patients in occupational disability compensation systems have been noted for having persistently poor outcomes following standard treatment modalities (DeBerard, Masters, Colledge, Schleusener, \& Schlegel, 2001; Franklin, Haug, Heyer, McKeefrey, \& Picciano, 1994; Franklin et al., 2005; Maghout-Juratli, Franklin, Mirza, Wickizer, \& Fulton-Kehoe, 2006; Taylor, Deyo, Ciol, \& Kreuter, 1996).

The prevailing theoretical models of pain and disability, based on a biopsychosocial perspective, indicate that chronicity is associated with significant psychosocial barriers to recovery, poorer outcomes following standard care, and often require intensive multidisciplinary treatment modalities to address the complex interaction of various physiological, psychological, and socioeconomic factors (Franche \& Krause, 2003; Gatchel, 1991, 1996; Krause \& Ragland, 1994). These theoretical models have also provided a reliable account of the phenomena of pain and disability among patients with occupational-related injuries, especially in accounting for the 
psychosocial barriers prevalent within the disability compensation setting. Additionally, these theoretical models clearly delineate stages or phases of pain and disability, and underscore the practical implications of a painful condition that progresses to chronic stages. One of these implications involves the cost of dealing with patients who are in the late stages of a chronic pain condition. Given the complex nature of chronic pain, reviews of the literature have unsurprisingly documented that intensive, interdisciplinary treatments based on the biopsychosocial model of pain are vastly more cost-effective than standard single-discipline treatment modalities, even if the upfront cost of the interdisciplinary treatment is greater than standard care (Gatchel \& Okifuji, 2006; Turk, Loeser, \& Monarch, 2002). However, despite the clear implications of chronicity and the documented amounts of staggering costs associated with pain, there has been very little research on the link between cost and outcomes for early rehabilitation for chronic pain conditions, relative to late rehabilitation.

The present study, therefore, was designed to investigate the cost-effectiveness of early rehabilitation for chronic pain and disability. The study utilized a cohort of patients who received functional restoration for chronic disabling occupational musculoskeletal disorders (CDOMD) following a work-related injury within the Workers' Compensation system, between years 1998 and 2005. Patients were classified according to duration of disability, resulting in three groups corresponding to Early Rehabilitation (4 - 8 months of disability), Intermediate Duration (9-18 months of disability), and Delayed Rehabilitation (> 18 months of disability). Groups were compared on treatment outcomes, utilizing validated measures of functional status and 
psychosocial well-being, as well as on objective socioeconomic outcomes documented at one-year post-rehabilitation. Additionally, a full economic evaluation was conducted incorporating both cost-effectiveness and cost-utility approaches of economic analysis. Finally, all relevant costs over the duration of disability were quantified in order to provide an overall cost-of-illness estimate for the cohort, including medical costs, disability benefits, and productivity losses.

Prior to introducing the study, a thorough literature review is presented on the issues related to chronic pain and disability. The literature review section begins with a review on the major theoretical viewpoints of pain, underscoring the conceptual differences between acute and chronic stages of pain. This will be followed by a review of present guidelines for treatment of pain, delineating the levels of care that are needed for the increasing complexity of the various stages of pain and its resulting disability. Next, the major economic theories that are the foundation of economic evaluations is presented, followed by a discussion of the application of these theoretical perspectives in healthcare research. Following this, a review of the various tools and methodology related to economic evaluations are presented. The application of economic evaluation methods to evaluate the cost-effectiveness of biopsychosocial-based treatment modalities for pain is discussed next. Finally, a discussion on the conceptual and contextual basis for the formulation of the study is presented, followed by presentation of the study design, methodology, results, and a discussion of the findings. 


\section{Theories of Pain}

\section{Biomedical Reductionism}

This theory is the earliest formulation of pain within the medical field, dating

back to the $17^{\text {th }}$ century. The central assumption within this theory was that pain is a consequence of specific physiological damage or impairment (Turk \& Monarch, 2002).

This damage or impairment to tissue or body part was assumed to be identifiable through objective tests or observations. This assumption proved to be initially successful and resulted in tremendous advancements in medical technology. As a result, specific treatment modalities were developed to correct or alleviate the pathology. However, over time, systematic research revealed that, given the same pathology, patients' self-reports of pain and its consequent disability varied considerably. A similar problem was the diverse responses among patients given the same treatment modality for a given pathology. The paradox that emerged from this discrepancy resulted in the general consensus that the relationship between self-report of pain and pathology is only moderate at best (Flor \& Turk, 1988; Turk \& Monarch, 2002; Waddell \& Main, 1984). Accompanying the perception of pain is usually a myriad of other factors, such as fear, anxiety, and sleep disturbances. These are usually exacerbated in conditions when pain becomes chronic, and can result in poorer psychosocial functioning and depression. However, all these factors were viewed as a consequence of pathology and were thus considered secondary factors (Turk \& Monarch, 2002). The underlying assumption was that, if the pathology was cured or corrected, these consequential factors should also cease to exist. This was known as somatogenic pain. In reality, 
though, many medical conditions arise where severe pain is reported but no specific pathology can be identified. These include conditions like chronic widespread pain and fibromyalgia, temporomandibular disorders, and chronic back pain (Aaron, Burke, \& Buchwald, 2000; Epstein et al., 1999; Gatchel, 2002; Gatchel, Stowell, Wildenstein, Riggs, \& Ellis, 2006; Gracely, Petzke, Wolf, \& Clauw, 2002; Greene, 2001; Gremillion, 2000; Kight, Gatchel, \& Wesley, 1999). When no pathology could be reliably identified, the disorder was termed psychogenic pain (i.e., the pain has a psychological basis). This dichotomous classification of pain, and its consequent disability, persisted in the field of medicine until relatively recent times.

\section{Gate Control Theory of Pain}

This theory by Melzack and Wall (1965) revolutionized the understanding of pain by implicating the role of psychological factors in the perception of pain. The two major contributions of the gate control theory (GCT) were the modulation of the perception of pain by interacting neurons, and the implication of the central nervous system in processing nociception. At the level of the peripheral nervous system, afferent nociceptive nerves are responsible for sending signals to the central nervous system via ascending pathways. These neurons consist of at least two types of fibers: the $A \delta$ fibers responsible for rapid signaling of intense, acute pain; and the $C$ fibers for chronic and throbbing type of pain (Melzack \& Wall, 1965). These two afferent nociceptive nerves can be inhibited by non-nociceptive nerves consisting of the $A \beta$ fibers. The GCT identified the dorsal horn area of the spinal cord as one area where pain transmission is modulated by interacting neurons. These areas in the dorsal horn receive input from the 
nociceptive and the non-nociceptive fibers. The non-nociceptive fibers, when activated, inhibit the firing of the nociceptive fibers in response to some external stimulus, thus "closing the gate" and preventing transmission of pain signals to the brain. In addition, the nociceptive fibers, when activated, can also function as an inhibitor of the nonnociceptive fibers and result in "opening the gate", thereby increasing the likelihood of pain signals being transmitted to the brain. The GCT also identifies a region of the brain responsible for modulating the transmission of pain signals. For example, the periaqueductal grey matter, when stimulated, can inhibit nociceptive neurons that converge in the spinal cord, via a descending pathway, thus reducing the probability of pain signals being transmitted to the brain (Melzack \& Casey, 1968).

These physiological mechanisms involved in nociception and subjective perception of pain resulted in several theoretical implications (Turk \& Monarch, 2002). Firstly, the central nervous system is implicated as a vital component in understanding pain and its related consequences. Secondly, the dichotomy of somatogenic and psychogenic pain was disproved; the GCT implies that both psychological and physiological factors can modulate the subjective experience of pain. And thirdly, the GCT implies that merely correcting or blocking the physiological pathways implicated in pain perception would be inadequate in totally eliminating perception of pain.

\section{Biopsychosocial Model of Pain}

The GCT opened avenues for the formulations of broad and more comprehensive models to explain not only pain, but diseases in general. The biopsychosocial approach to medicine in general was first formulated by Engel (1977). 
Central to the biopsychosocial perspective is the distinction between disease and illness. Whereas disease represented specific pathology that could be objectively identified, illness corresponded to the subjective experience of the disease, or its broader manifestation beyond identifiable pathology (Gatchel, 2004; Turk \& Monarch, 2002). The pathology serves as a stimulus which is then moderated by the individual's present psychological status, experience, genetic predispositions, and social and cultural factors. The manifestation of illness is thus dependent upon these interacting factors.

Engel's general model of illness identifies distress as the first response to pathology. Distress over the physical problem can be conceptualized as the subjective experience of the pathology. This subjective experience can then lead to emotional responses, characterized by illness behavior. Depending on the type of pathology and the individual itself, these illness behaviors can take on a variety of forms, such as fear, anxiety, or depression. When the pathology becomes chronic, the illness behaviors will eventually lead to the adoption of the "sick role", which is the specific set of behaviors an individual adopts to minimize distress.

In applying this general model in the formulation of a model of pain, Loeser (1982) identified nociception as the basic marker for pathology, which then leads to the subjective experience of pain. Pain would then lead to a range of emotional responses, broadly defined as suffering. If the nociception persists, and suffering becomes chronic in response to the subjective experience of pain, an individual would then start exhibiting pain behaviors. These behaviors, analogous to the sick role, may include such behaviors as avoiding activity due to the fear of triggering pain (Gatchel, 2004). 
This biopsychosocial model is also consistent with the GCT in that these specific factors identified within the model can interact, and there is not only a one-way progression from pathology to manifestation of illness. This key concept of the biopsychosocial model can be clearly identified in a discussion of the progression of pain from the acute to the chronic stage.

\section{Development of Pain: From Acute to Chronic}

The three-stage model of progression is, to date, the most comprehensive description of the development of pain from the acute to the chronic stage (Gatchel, 1991, 1996). Stage 1 describes the acute phase of pain, and involves basic responses to the perception of pain. These responses include a range of normal emotional reactions such as fear, anxiety, and worry. Such emotional responses serve as a protective function that signals to the individual that some sort of attention may be required to prevent further tissue damage or the development of a complicated medical condition. The duration of Stage 1 is dependent upon the normal healing period for most painful conditions underlying some sort of pathology, and can range from between two weeks to four months. In general, pain lasting for more than four months begins to develop into chronic pain.

Stage 2 of this model marks the beginning of chronicity and involves the exacerbation of physiological and psychosocial conditions. At the physiological level, physical deconditioning starts to develop (Mayer, Gatchel, Porter, \& Theodore, 2006; Mayer \& Press, 2005). For example, if the pain is due to injury of a certain body part, an individual's behavior is geared towards avoiding utilization of the injured body part 
as much as possible for fear of increased pain or re-injury. This results in physical deconditioning. Additionally, the symptoms in this Stage manifest themselves according to the diathesis-stress perspective. At this point in the progression of pain, the stress of coping with pain leads to the exacerbation of underlying psychological characteristics within a given individual. These underlying characteristics include predisposing psychological variables, such as the individual's personality and general psychosocial well-being, as well as external factors such as socioeconomic and environmental factors. Characteristic responses at this Stage include learned helplessness, anger, and distress. These affective consequences of chronic pain can perpetuate and even exacerbate the perception of pain (Fernandez, 1998), which then leads to a pain-stress cycle and increased somatization (Gatchel \& Oordt, 2003). Development of comorbid psychopathology also occurs at this Stage, including personality disorders, psychophysiological disorders, major depressive disorders, and substance abuse disorders (Dersh, Gatchel, Mayer, Polatin, \& Temple, 2006; Dersh, Mayer, Theodore, Polatin, \& Gatchel, 2007; Dersh et al., 2007; Dersh, Polatin, \& Gatchel, 2002).

If chronicity is allowed to develop, progression into full-blown chronic pain takes place. Stage 3 of the model characterizes this stage of pain as a complex interaction among physiological, psychological, and social processes, and the individual becomes preoccupied with the pain. This Stage is analogous to the sick role as discussed in the general biopsychosocial model above (Engel, 1977). In addition to the focus on pain, the individual at this Stage exhibits poor social and occupational 
functioning, and begins to develop secondary gain issues (Aronoff et al., 2007; Dersh, Polatin, Leeman, \& Gatchel, 2004; Fishbain, 1994; Leeman, Polatin, Gatchel, \& Kishino, 2000). Such behaviors are characterized by the avoidance of responsibilities, and seeking out financial compensation for the pain. These secondary gain issues then begin to serve as reinforcers that maintain maladaptive behaviors (Gatchel \& Oordt, 2003), which then result in complete physical and psychological deconditioning.

The key concept then, in any type of medical setting dealing with pain, is to prevent the development of Stage 3 chronic pain. Pain is best addressed at early stages to prevent the synergistic effect of several interacting and confounding physiological, psychological, and social factors. Consistent with this three-stage model of pain and the advances in the theory of pain, a formal biopsychosocial model for treating pain has been developed. This model involves levels of care that are commensurate with the level of progression of pain. These levels of care within a biopsychosocial paradigm are discussed next.

\section{Biopsychosocial Rehabilitation}

Biopsychosocial rehabilitation can be broadly categorized into three levels of care: primary care, secondary care, and tertiary care (Gatchel \& Turk, 1996). These levels of care are characterized by a biopsychosocial approach to dealing with pain, and are differentiated primarily by intensity and the comprehensive nature of the treatment modalities. It should be noted, however, that pain patients need not necessarily progress through the levels of care in a sequential manner. A stratified approach is suggested for 
matching the stage of chronicity with the appropriate level of care required (Von Korff, 1999).

\section{Primary Care}

This level of care is designed to address pain and its related issues at the acute stage. The underlying aim of primary care is to control the pain symptoms. In addition, primary care treatment modalities are geared towards promoting the recovery of the pathophysiology and prevention of physical deconditioning (Mayer \& Press, 2005). Treatment modalities that accomplish these goals include medication, thermal application, immobilization of injured joints, bed rest, and traction. Psychosocial issues are routinely addressed at the primary care level, and these include identification of any barriers to recovery such as fear and anxiety about the pain (Theodore, Chan, \& Gatchel, 2008). Psychosocial interventions at this stage are therefore aimed at reassuring the patient that the pain symptoms are temporary and will soon be alleviated, given adherence to the treatment modality and compliance with any medication regimen.

Secondary Care

This level of care is targeted towards patients at the post-acute stage of pain who are showing signs of functional limitations due to pain, as well as the development of psychosocial barriers to recovery. The main goals of secondary care are to promote reactivation of the underlying physiology and the prevention of long-term physical and psychological deconditioning (Mayer \& Press, 2005). Treatment approaches in secondary care should ideally include an interdisciplinary healthcare team consisting of 
the primary care physician, clinical psychologists, physical therapists, and nurses or health educators. If one of the goals of rehabilitation include a return to previous occupational status, then an occupational therapist would also be a useful component of the rehabilitation setting (Mayer et al., 2003; Theodore, Chan, \& Gatchel, 2008).

Treatment modalities at this level include structured exercise programs, functional training for improving general health and work capacity, and cognitivebehavioral interventions designed to address psychosocial barriers to recovery that play a role in the development of chronicity. Several randomized controlled trials (RCTs) evaluating an interdisciplinary secondary care approach have documented evidence for highly satisfactory outcomes, including increased reduction of pain, improved general health and self-efficacy, increased treatment satisfaction, lower healthcare costs, as well as satisfactory resumption of occupational status and activities of daily living (Hagen, Eriksen, \& Ursin, 2000; Indahl, Haldorsen, Holm, Reikers, \& Holger, 1998; Karjalainen et al., 2004; Karjalainen et al., 2001; Lindstrom et al., 1992).

\section{Tertiary Care}

The main goal of tertiary care is to prevent permanent disability due to pain. At this stage, the emphasis is on managing pain and its consequent disability (Mayer \& Press, 2005). Patients who end up in tertiary care have either not responded well to primary or secondary care, or have been evaluated by the primary care physician as having a complicated medical case that is consistent with the earlier reviewed Stage 3 chronic pain. In addition, this level of care is usually the final step after patients have exhausted all other surgical and conservative approaches for dealing with the pain. 
Given the complex nature of interactions among several factors within Stage 3 of chronic pain, treatment modalities at this level of care are necessarily more complex and require a multidisciplinary approach. Tertiary care is primarily tailored towards the individual patient, and the healthcare team consists of clinical psychologists or psychiatrists, physical therapists, occupational therapists, disability case managers, and nurses or health educators, in addition to the primary physician.

General treatment modalities are targeted towards reversing physical deconditioning via re-activation of the affected physiology, coping with limited pain and disability, addressing of comorbid psychopathology, and the removal of psychosocial barriers such as secondary gains (Deschner \& Polatin, 2000; Mayer et al., 2003; Mayer, Gatchel, Porter, \& Theodore, 2006). This level of care can take two broad approaches, differentiated by the ultimate goal of rehabilitation. Palliative pain management is a lower intensity treatment approach with the goal simply being the management of pain. Pain-relieving narcotics and medication for psychiatric comorbidity are the usual treatment modalities. Additionally, psychological interventions aimed at improving coping techniques and to decrease pain and stress are provided to help patients deal with a lifestyle of reduced function (Mayer \& Press, 2005).

The second type of approach to tertiary care includes higher intensity treatment modalities with the goal of preparing chronically disabled patients for resumption of occupational status, in addition to managing pain and disability. These tertiary care rehabilitation approaches include intensive, interdisciplinary modalities such as 
functional restoration (Mayer et al., 1985), and also general return-to-work programs (Li, Li-Tsang, Lam, Hui, \& Chan, 2006). In addition to the general tertiary care treatment modalities discussed above, these rehabilitation approaches may also involve narcotic detoxification, structured graded exercises aimed at improving functional capacity, work hardening and skills training aimed at improving work capacity and employability, disability and occupational case management, and individual placement and support for returning to work (Mayer \& Press, 2005).

A large body of evidence has been systematically reported in the literature over the last two decades documenting the treatment efficacy of the functional restoration approach to managing chronic pain in the CDOMD patient population. Objective oneyear post-rehabilitation outcomes have been obtained from cohort studies, as well as RCTs, and include: increased resumption of active occupational status and activities of daily living; decreased health care utilization; reduced levels of pain intensity; improved readiness to change; improved psychological well being; and, resolution of outstanding medico-legal issues (Becker, Sjogren, Beck, Olsen, \& Eriksen, 2000; Guzman et al., 2001; Hazard et al., 1989; Mayer et al., 1985; Patrick, Altmaier, \& Found, 2004). The highly satisfactory results of functional restoration have been shown to be temporally stable (Mayer et al., 1987), and can be generalized across different socioeconomic and medico-legal systems (Bendix et al., 1996; Corey, Koepfler, Etlin, \& Day, 1996; Hildebrandt, Pfingsten, Saur, \& Jansen, 1997; Jousset et al., 2004). Finally, it has been demonstrated that functional restoration, along with other interdisciplinary approaches to pain management, are more cost-effective than standard conservative treatment 
(Gatchel \& Okifuji, 2006; Skouen, Grasdal, Haldorsen, \& Ursin, 2002; Turk, 2002; Turk \& Okifuji, 1997).

\section{Application of Economic Theories in Healthcare}

The major goal of health economics is to provide a foundation for decisionmaking in the allocation of resources in the healthcare system (Garber, Weinstein, Torrance, \& Kamlet, 1996). There are several tools that have been developed for use in economic analyses in general, and these are also applicable for analyses within healthcare settings. Each of these tools have their own sets of assumptions, specific methodology, types of conclusions that can be inferred from data, as well as inherent advantages and disadvantages. However, all these tools are based upon well-established theories in economics. Two economic perspectives relevant to the healthcare setting are discussed, along with their respective implications for methods of economic analysis in healthcare research.

\section{Theoretical Foundations}

Welfare economics is the predominant theory applied in economic evaluations. This economic theory is based on the concept of welfarism, which posits that consequences resulting from a specific course of action (e.g., policies, interventions, laws), when significant, are an impact on overall human welfare (Kaplow \& Shavell, 2002). Therefore, any course of action needs to be evaluated based on its actual or potential consequences. Within the context of welfare economics, the general goal is to assess the desirability of alternative allocation of resources for different courses of action (Garber, Weinstein, Torrance, \& Kamlet, 1996). Each given alternative course of 
action may incur different costs and provide for varying returns (or benefits) to society at large. Therefore, assessments of these alternatives need to incorporate a societal point of view, as opposed to individual- or group-centered benefits. However, the overall societal benefit is ultimately a function of some maximum benefit derived from quantifying desirability (for a given state) at the level of the individual (Arrow, 1963).

In order to extend individual desirability to a larger construct of societal benefit, two assumptions need to be met (Garber, Weinstein, Torrance, \& Kamlet, 1996). The first assumption states that individuals seek to maximize a well-defined preference function that reflects their overall sense of well-being, or expected utility. The second assumption states that overall societal welfare is an aggregation of these individual preferences. The concepts of a preference function and utilities themselves arise from earlier work on the expected utility theory (von Neumann \& Morgenstern, 1944). This seminal work in the field of decision-making describes the process by which an individual makes a decision under conditions of uncertainty: the utility (quantitative representations of preference) associated with the outcomes of each possible alternative is evaluated and combined as a weighted average, based upon the probability of the outcomes within each alternative course of action. The most desirable (and rational) action is the one associated with the highest expected utility. Therefore, given a set of alternative actions, an ordinal ranking of each action and their consequent outcomes can be generated. The overall underlying assumption in this theory is that people make rational choices, which is often not the case in reality (Kahneman \& Tversky, 1979). 
A second school of thought that branched out of welfarism is the concept of extra-welfarism (Culyer, 1989). Whereas welfarism requires that actions be evaluated on the basis of their overall impact on welfare, extra-welfarism dictates that the action toward achieving a particular objective be evaluated on the basis of the relevant targeted outcomes, rather than being evaluated relative to overall welfare. This distinction is best captured in its application in healthcare research, in that extra-welfarism seeks not to maximize overall expected utility, but to maximize health-related utility (Gyrd-Hansen, 2005). It is this school of thought that provides the theoretical grounds for modern-day economic analysis in healthcare research, specifically on the development and implementation of the concept of health-related utilities such as the concept of QualityAdjusted Life Years (QALYs). However, there have been suggestions that there should not be any focus on utilities per se but rather evaluating healthcare outcomes on the basis of functioning and capabilities relevant to health modalities of concern (Sen, 1997a, 1997b). While this approach has the advantage of providing context-relevant outcomes (Richardson \& McKie, 2005), a major disadvantage is that there are too many individual outcomes that encompass health-related contexts (Gasper, 1997), thus limiting comparability across different types of alternatives for purposes of decisionmaking in regards to resource allocation. For now, although economic analyses in healthcare research do incorporate variations of health-related utilities as a measure of standardized outcome, considerable debate is still ongoing on the absolute monetary worth of these outcomes (Fryback \& Lawrence, 1997; Gyrd-Hansen, 2005; Johannesson, 1995). 
Applications in Healthcare Research

In the domain of healthcare research, significant application of the principles of welfare economics have been used in the construction of composite health-related quality of life measures, most notably the concept of the Quality-Adjusted Life Year (QALY). The QALY was first developed by Klarman, Francis and Rosenthal (1968) in a study on chronic renal failure, and subsequently refined and standardized for use as an overall health outcome suitable for economic evaluations of healthcare interventions (Gold, Siegel, Russell, \& Weinstein, 1996). The QALY is basically a composite measure that incorporates gains in quality of life related to reduced morbidity and reduced mortality. The specific types of health-related quality of life measures utilizing the QALY are discussed in a subsequent section, and only the application of expected utility theory is explained here. In the case of health-related quality of life measures based on the concept of utilities, each outcome or health state is defined by a preference (or utility) weight. The idea of a quality-adjusted life year, then, is simply the preference weight for the given health state multiplied by the time spent in that health state. Ideally, these weights should be elicited based on population- or communitybased samples to be consistent with the societal perspective of welfare economics (Gold et al., 1996).

Methods for eliciting these preference weights can be done in one of two general ways (Gold et al., 1996). The first way is based on the axioms and methods of expected utility theory. It involves either the standard gamble approach or the time-tradeoff approach. Standard gamble involves giving individuals a choice between certainty of 
continued life in a specific less-than-optimal health state, and a gamble with two possible outcomes (Torrance, 1986). One outcome of the gamble is death while the other outcome is a state of full health, with utility values of 0 and 1 respectively. The standard gamble approach involves systematically altering the probabilities of the outcomes in the gamble until the individual expresses indifference between the choice of certainty of the given health state and the gamble. At the point of indifference, the expected value of the gamble is designated as the utility weight for the given health state, relative to full health and death. In contrast, the time-tradeoff approach involves two certain outcomes consisting of a better and a poorer health state (Torrance, Thomas, \& Sacket, 1972). An individual is asked to specify the amount of time, in life-years, willing to be sacrificed to be in a better health state versus the poorer health state. The method begins with presenting the alternative of being in a poorer health state for a longer period of time followed by death, and an alternative of being in a better health state for a shorter period of time followed by death. The time period in the better health state is varied until the individual expresses indifference between the alternative health states. The utility weight at the point of indifference is calculated based on life expectancy under the better health state divided by the life expectancy in the poorer health state.

A second less complicated method in obtaining utility weights is through the use of rating scales based on either categorical choices or a visual analog scale for preference of a given health state, along a continuum of death and perfect health (Gold et al., 1996). Though less of a burden on participants, and provides ease of data 
collection compared to the expected utility approach, rating scales were initially criticized for a range of issues, including being relatively more subjective (Richardson, 1994), does not take into account the underlying difficulty in enumerating one's feelings for hypothetical representation of a given health state (Patrick, Starks, Cain, Uhlmann, \& Pearlman, 1994), and suffers from low test-retest reliability at one-year intervals (Torrance, 1976). To make matters worse, different measurement techniques, including comparisons between psychophysical and expected utility approaches, have been reported to have discrepant weights for the same health state (Gold et al., 1996).

Nevertheless, rating scales are still the most widely used method for generating utility weights (Garber, Weinstein, Torrance, \& Kamlet, 1996). In arriving at a consensus on this issue, the International Panel on Cost-Effectiveness in Health and Medicine, convened by the U.S. Public Health Service in 1993, concluded that the "choice of preference measurement method should be based on the decision or problem to be solved, the practical considerations involved in the study, and the use to which data will be put." (Gold et al., 1996, p. 118). Furthermore, the panel contends that discrepancies in utility weights between the two approaches may simply be a consequence of variation in elicitation techniques, rather than being due to any departure from the underlying theoretical foundation of economic analysis, i.e. to obtain the societal perspective of preference for given health states (Gold et al., 1996).

However, since the panel's report in 1996, the earlier criticisms about the psychometric qualities of QALY rating scales have been addressed in the development of modern utility-based health-related quality of life measures, or by revisions of 
previously developed measures. Published studies on health-related utility rating scales during the decade following the panel's report, have demonstrated stable psychometric properties and good concurrent validity with existing and well-validated generic and disease-specific health-related quality of life instruments (Brazier, Roberts, \& Deverill, 2002; Brazier \& Roberts, 2004; Fryback, Lawrence, Martin, Klein, \& Klein, 1997;

Pickard, Wang, Walton, \& Lee, 2005; Solberg, Olsen, Ingebrigtsen, Hofoss, \& Nygaard, 2005).

\section{Costs and Outcomes in Economic Evaluations}

\section{Valuation of Costs}

In the strictest sense of welfarism, every component and consequence of an alternative under investigation can technically be classified as a cost, and therefore expressed in monetary units. For economic evaluations within the healthcare setting, associated costs can be broadly categorized into medical costs, non-medical costs, productivity losses, and intangible costs (Haddix, Corso, \& Gorsky, 2003; Luce, Manning, Siegel, \& Lipscomb, 1996).

Depending on the nature of the intervention, medical costs may include any screening and diagnostics, specific surgical or non-surgical intervention, hospitalization costs, medication, and visits to a healthcare provider (Haddix, Corso, \& Gorsky, 2003). In evaluations of multimodal intervention procedures, a common valuation of this direct cost is often conceptualized as the hours or units of treatment billed (Critchley, Ratcliffe, Noonan, Jones, \& Hurley, 2007; Hatten, Gatchel, Polatin, \& Stowell, 2006; Rivero-Arias et al., 2005; Rivero-Arias, Gray, Frost, Lamb, \& Stewart-Brown, 2006). 
Non-medical costs involve direct costs consequent to the intervention, but otherwise not classified under any type of medical modality. Depending on the scope of the evaluation and the nature of the intervention, the most common non-medical costs may include transportation costs involved with travelling to receive the intervention, any requirement of home-based care, childcare costs, or dietary prescriptions (Haddix, Corso, \& Gorsky, 2003).

Productivity losses involve any costs associated with the patient's inability to engage in occupational activities, leisure activities, or activities of daily living during the period of intervention and also any period following the intervention. The most common cost associated with productivity losses is the patient's time spent receiving the intervention. Evaluating this specific cost is critical for comparisons of interventions that differ in intensity and treatment duration (Haddix, Corso, \& Gorsky, 2003; Luce, Manning, Siegel, \& Lipscomb, 1996), but can be omitted if the treatment duration is negligible or the various alternatives have similar time demands (Luce, Manning, Siegel, \& Lipscomb, 1996). Two other major cost estimates included in productivity losses involve morbidity and mortality costs (Luce, Manning, Siegel, \& Lipscomb, 1996). Morbidity costs are associated with any loss in time or ability to perform occupational or leisure activities following the intervention (e.g., recuperation time, if interventions being investigated are surgical options). Mortality costs are associated with changes in life expectancy as a result of the intervention. However, it should be noted that while productivity losses due to both morbidity and mortality costs can sometimes be accurately represented in monetary units, the impact of morbidity and 
mortality are recommended to be included as part of the "effectiveness" component of economic evaluation, expressed in the form of outcomes rather than in monetary units (Luce, Manning, Siegel, \& Lipscomb, 1996).

Finally, intangible costs refer to any type of costs associated with the intervention or outcomes that are often difficult to express in monetary units. Within the healthcare setting, intangible costs refer to resulting differences among the interventions being investigated on constructs such as pain, psychological deficits, and emotional distress (Haddix, Corso, \& Gorsky, 2003). Although in the strictest sense of welfarism, these are classified as costs, economic evaluations within the healthcare setting include these intangible costs as part of the "effectiveness" component of economic evaluations in the form of self-report outcomes (Haddix, Teutsch, \& Corso, 2003; Luce, Manning, Siegel, \& Lipscomb, 1996).

\section{Outcome Measures}

Outcomes of a given healthcare intervention are often conceptualized as the "effectiveness" component of economic evaluations. Therefore, outcomes form the basis of evaluating the effectiveness of one intervention or strategy relative to another. Outcomes can be broadly generalized into two categories. The first of these are naturally occurring outcomes relevant to the healthcare intervention under study. These may take the form of objective outcomes, such as lives saved, disease averted, resumption of occupational activity, and reduction of healthcare consumption (Yabroff \& Mandelblatt, 2003). Alternatively, outcomes can also be conceptualized as measures of constructs such as pain, function, psychosocial well-being, and overall quality of life 
(Yabroff \& Mandelblatt, 2003). As noted previously, a significant disadvantage of such natural outcomes is that they may only be applicable to a given intervention, and fail to provide an accurate representation of effectiveness when another intervention utilizes a different type of outcome. Additionally, economic evaluations conducted to facilitate decision-making for priorities among different types of treatment modalities will be difficult when faced with a diversity of outcomes, rather than a single, multidimensional construct of effectiveness (Dasbach \& Teutsch, 2003; Yabroff \& Mandelblatt, 2003).

To overcome these disadvantages mentioned above, a second category of outcome measuring overall quality of life is routinely included in economic evaluations. Specifically, this measure exclusively refers to the concept of the Quality-Adjusted Life Year (QALY). As discussed in the previous section, QALYs are measured using utilitybased, self-report instruments. There are a variety of utility-based, psychometricallysound, self-report instruments developed specifically for the measurement of QALYs in economic evaluations within healthcare settings. These are primarily based on generic health-related domains that measure some combination of social function, psychological function, and physical function (Gold et al., 1996). The most widely used instruments validated across multiple disease populations include the EuroQol EQ-5D (Kind, 1996), the Health Utility Index - Mark 3 (HUI-3)(Feeny et al., 2002), and the Quality of WellBeing Scale (QWB)(Kaplan \& Anderson, 1993). These instruments are based on categorical choices for a specific health state within a given domain (HUI-3 and QWB), or rated using a visual analog scale (EQ-5D). In both cases, all responses across a variety of constructs tapping into health-related quality of life are scored and combined 
as a utility score ranging from 0 to 1 , corresponding to death and perfect health respectively (Dasbach \& Teutsch, 2003).

While there has been an increase in the use of these instruments over the last decade, especially given the growing importance of economic evaluations using healthrelated utility as the outcome measure, most research protocols usually collect generic profile-based quality of life measures that are not based on utility scores, such as the Short-Form 36 (SF-36)(Ware, Snow, Kosinski, \& Gandek, 1993) or Short-Form 12 (SF12)(Ware, Kosinski, \& Keller, 1996). However, several quantitative applications have been developed to convert generic profile-based (non-utility) quality of life measures into utility scores based on the common utility-based instruments. For example, validated conversion algorithms based on the EQ-5D, HUI-3, and QWB have been developed to convert SF-36 and SF-12 profile-based scores into health-related utility scores (Brazier, Roberts, \& Deverill, 2002; Brazier \& Roberts, 2004; Franks, Lubetkin, Gold, \& Tancredi, 2003; Franks, Lubetkin, Gold, Tancredi, \& Jia, 2004; Fryback, Lawrence, Martin, Klein, \& Klein, 1997; Lawrence \& Fleishman, 2004). Reviews of these various algorithms applied to diverse clinical populations have reported satisfactory predictive validity, concurrent validity and face validity (Hollingworth et al., 2002; Pickard, Wang, Walton, \& Lee, 2005), thus providing an avenue for QALYbased economic evaluations in research settings that have not implemented data collection of utility-based measures. 
Analytical Methods for Economic Evaluations

Several different analytical methods have been derived from the theoretical foundations of economics, for application within healthcare settings. These different types of methodology can be delineated into partial or full economic evaluations. Methodological issues that are common to the various types of economic evaluations are discussed, followed by a review of the three major types of methodology for economic evaluations.

Partial versus Full Economic Evaluation

Economic evaluations can be classified into two broad categories (Drummond, O'Brien, Stoddart, \& Torrance, 1997). Full economic evaluations include Cost-Benefit Analysis, Cost-Effectiveness Analysis, and Cost-Utility Analysis. It is these three methods that are conventionally referred to as economic analysis, and these will be discussed in detail in the following sections. Partial economic evaluations consist of descriptives and/or comparisons of either costs or outcomes, and depending on the focus of the study, can be classified as Cost Description, Outcome Description, Efficacy or Effectiveness Evaluation, Cost Analysis, or Cost-Outcome Description. None of these partial evaluations describe and analyze the link between costs and outcomes (or effectiveness), but are usually reported as part of full economic evaluations in terms of comparing treatment groups or different interventions on the basis of individual cost and outcome variables (Drummond, O'Brien, Stoddart, \& Torrance, 1997; Vetter, 2007). 
Common Characteristics Across Methods of Economic Evaluation

All analytical methods relevant to economic analysis share some common characteristics in their design and execution. Once the research question is framed and the study population identified, the first step in designing any economic analysis is to determine the perspective of the study (Farnham \& Haddix, 2003). The welfare economic paradigm dictates that a big-picture societal perspective is utilized, i.e., all relevant costs and benefits be accounted for in the economic analysis regardless of who pays the cost and who receives the benefit. For example, long-term costs or outcomes following the intervention should also be factored into the economic evaluation, and not just those that are concurrent with the timeframe of the intervention period. An additional component of the societal perspective is the use of QALYs as the outcome measure. This is primarily because health-related utility weights are generally developed by sampling community-based preferences for health states (Gold et al., 1996). While all these may not always be feasible in practice within the scope of healthcare research, present guidelines for economic evaluations within the healthcare setting recommend adopting a societal perspective as best as the availability of data allow (Gold, Siegel, Russell, \& Weinstein, 1996). The reason for this is because the societal perspective, ideally, provides for comparability among different types of healthcare interventions without being distorted by narrow and limited interests. Other perspectives utilized in economic evaluations ultimately depend on the objectives of the economic evaluation, and can include a third party payer perspective, healthcare provider perspective, governmental agency perspective, and the patient perspective 
(Farnham \& Haddix, 2003). In these other perspectives, the costs and outcomes included in the analysis are limited only to those relevant to the party concerned.

The next step to be considered in the study design is the analytical time frame of the study (Farnham \& Haddix, 2003). The time frame begins at the starting point of the healthcare intervention under study, and extends for a duration of time post-treatment that allows for sufficient and reliable documentation of intervention outcomes. The length of time that the analytical horizon extends to ultimately depends on the healthcare intervention being evaluated. For example, healthcare interventions that include patient survival rates as the major outcome, e.g. interventions for cardiovascular diseases, often have a time span of multiple years following the intervention (Mihaylova et al., 2005). On the other hand, evaluations of chronic pain interventions usually have shorter analytical horizons. For example, a recent review of economic evaluation performed for chronic back pain interventions reported that approximately $80 \%$ of published studies between years 1988 and 2006 incorporated an analytical horizon limited to 1-year post-treatment or less (Vetter, 2007).

In addition, at least one relevant comparator intervention should be specified as part of the analysis (Farnham \& Haddix, 2003; Gold, Siegel, Russell, \& Weinstein, 1996). The comparator may vary depending on the objectives of the study, and can include a no-treatment option, an expanded or reduced treatment option, current standard care, different treatment strategy, or a different treatment modality. The primary purpose of a comparator in the economic evaluation is to facilitate the calculation of incremental costs and outcomes of one treatment, relative to the other. 
Finally, once the data is collected and the primary analyses concluded, all economic evaluations should be subject to secondary analyses in order to evaluate any sources of uncertainty in the initial analysis (Gold, Siegel, Russell, \& Weinstein, 1996). This is simply due to the fact that economic evaluations are very rarely implemented without some type of indirect estimation of costs or outcomes, or with assumptions that are not backed by strong evidence in the research literature. When economic evaluations are based solely on estimates of costs and outcomes synthesized from literature review (e.g., from meta-analyses), uncertainty can be evaluated using sensitivity analysis. This is conducted by systematically varying the values of the initial estimates, based upon the range of values reported in the literature, and observing their impact on the costs and outcomes within the summary statistics relevant to the different types of evaluation tools (Goldie \& Corso, 2003). For economic evaluations based on raw data, statistical approaches to evaluating uncertainty are recommended, for example, by constructing confidence intervals for the summary statistics (Manning, Fryback, \& Weinstein, 1996), or reporting cost-effectiveness acceptability curves (Fenwick, Claxton, \& Sculpher, 2001). Other than the preceding four issues, each method has unique analytical principles, which in turn determine how results are interpreted and its scope of applicability to research in the healthcare setting. Three different methods of full economic evaluations are now discussed.

\section{Cost-Benefit Analysis}

The Cost-Benefit Analysis (CBA) method is the earliest form of an economic analysis, dating back to the mid $19^{\text {th }}$ century (Messonnier \& Meltzer, 2003), and is a 
direct application of the welfarist school of thought to economic evaluation techniques (Garber, Weinstein, Torrance, \& Kamlet, 1996). The defining characteristic of CBA is that both costs and outcomes are valued in monetary units. And, more importantly, the costs and benefits are valued with the widest perspective possible and not necessarily limited to variables that are closely related to the intervention or program being studied, i.e. it should take into account any long-term "ripple" effect associated with outcomes. The advantage of utilizing CBA is that, under ideal conditions, it provides seamless comparison of vastly different programs or interventions due to the objective nature of monetary valuations (Farnham \& Haddix, 2003). For example, in theory, a CBA can be used to compare a functional restoration program for chronic musculoskeletal pain in the Workers' Compensation setting, against a proposed Federal Government policy for increasing the number of airline baggage screeners at major airports in the United States. Thus, the CBA provides a comparability index that allows decision-making for priorities in allocation of resources.

In addition to direct costs and outcomes valued in monetary units, the CBA methodology takes into account both societies' total willingness to pay for the benefits and/or outcomes of a given intervention or program under study, as well as the opportunity costs or resources sacrificed to achieve these benefits or outcomes (Messonnier \& Meltzer, 2003). Results from a CBA are often presented as a summary statistic known as the Net Present Value (NPV) and the Benefits-Cost Ratio (BCR). The NPV involves adding the monetary value of all associated direct and indirect benefits, and subtracting from it the monetary value of all associated direct and indirect 
costs. When benefits outweigh costs, the NPV will be larger than $\$ 0$. On the other hand, the BCR is computed by dividing the monetary values of all benefits divided by the monetary value of all costs. A BCR greater than one implies that benefits outweigh the costs.

Despite the appealing nature of CBA in being able to provide comparisons based solely on monetary units, this method is the least preferred in economic evaluations within healthcare research. The biggest obstacle is the valuing of health outcomes in monetary units. There is often much controversy surrounding the valuation in monetary terms of a life saved, pain and suffering averted, functional improvements, and overall improvements in a person's health (Garber, Weinstein, Torrance, \& Kamlet, 1996; Messonnier \& Meltzer, 2003). Additionally, there is lack of standardization on the distinction between costs and benefits; i.e. benefits can also be expressed as negative costs, thus having a direct impact on the calculation of the Cost-Benefit Ratio (Messonnier \& Meltzer, 2003). For these reasons, CBA is rarely utilized in economic evaluations that are part of healthcare research. As Vetter (2007) reports in a review of economic evaluations applied to chronic pain interventions, only 2 out of 142 economic evaluations published from 1988 through 2006 utilized the CBA methodology.

\section{Cost-Effectiveness Analysis}

The Cost-Effectiveness Analysis (CEA) method is one of the two predominant economic evaluation methods utilized in healthcare research (Gift, Haddix, \& Corso, 2003). The other predominant method is the Cost-Utility Analysis, to be discussed in the next section. As reviewed by Vetter (2007), 30 of the 142 published economic 
evaluations for chronic pain interventions in the last two decades utilized the CEA method. This method is based on the extra-welfarist school of thought that recommends maximizing health outcomes as the focus of economic evaluations of healthcare interventions, rather than on traditional notions of utility that require health outcomes to be valued monetarily in a manner relative to overall societal welfare. As a result, the CEA does not value outcomes in monetary units, but instead reports outcomes and their associated effectiveness in the natural units relevant to the outcomes being investigated (e.g., mg/dl of cholesterol, unit decrease in pain scores, lives saved).

The CEA method provides for the calculation of two main types of costeffectiveness ratios as the summary statistic of the analysis (Gift, Haddix, \& Corso, 2003). In general terms, a cost-effectiveness ratio is basically the total or net costs of an intervention (in monetary units), divided by the effectiveness of the intervention measured in natural units of the relevant outcome. When a cost-effectiveness ratio is calculated by this method for each intervention being evaluated, it represents the average cost-effectiveness ratio for each individual intervention. To compare a pair of interventions, an incremental cost-effectiveness ratio (ICER) is computed by dividing the difference in monetary units of cost between two interventions by the difference in effectiveness of the two treatments measured in natural units of the outcome. The ICER represents the cost of one additional outcome of the intervention under consideration, relative to the comparator.

While this conceptualization of effectiveness provides for ease of measurement of effects that cannot be easily or even ethically described in monetary units, the 
limitation of this approach is that cost-effectiveness evaluations can only be compared to other interventions that utilize the same outcomes. As noted by Hatten, Gatchel, Polatin \& Stowell (2006), the unstandardized conceptualization of outcomes as measures of effectiveness results in problems generalizing the results of economic evaluations to similar patient populations that utilize a different set of outcomes. In order to facilitate comparisons in a standardized manner, a modification to the traditional CEA methodology has been developed and is based on health-related utility measures.

Cost-Utility Analysis

In terms of application to research in healthcare settings, the Cost-Utility Analysis (CUA) is among the two most predominant full economic evaluation methods. As reported by Vetter (2007), 29 of the 142 published economic evaluations for chronic pain interventions in the last two decades utilized the CUA method. However, the CUA is simply a special case of the CEA, and they are often referred to interchangeably (Farnham \& Haddix, 2003). Like the CEA, the CUA is also influenced by the extrawelfarist school of thought, but with the focus on maximizing health-related utility. Therefore, the CUA methodology differs from the CEA only in terms of the valuation of outcomes. Instead of quantifying outcomes in natural units, health-related utility measures are used, with the most common of these being the Quality-Adjusted Life Year (QALY). As discussed in the previous section, the QALY is a composite measure that incorporates gains in overall health-related quality of life. 
Similarly to the CEA, the CUA reports summary statistics in the form of costutility ratios. An average cost-utility ratio is simply the total cost of an intervention valued in monetary units, divided by the effectiveness of the intervention, valued as a health-related utility measure. Likewise, an incremental cost-utility ratio (ICUR) is computed by dividing the difference in monetary units of cost between two interventions by the difference in effectiveness of the two treatments measured in terms of health-related utility. These ratios correspond to the average cost per QALY gained by each individual intervention being studied, or in the case of the ICUR, the additional costs per QALY gained for one intervention relative to a comparator.

Based on the recommendations of the International Panel on Cost-Effectiveness in Health and Medicine (Gold, Siegel, Russell, \& Weinstein, 1996), health-related utilities should be incorporated as an outcome in any economic evaluation of healthcare interventions. The primary purpose for this recommendation is to provide a standardized conceptualization of health outcomes, which in turn can facilitate easier comparisons among different types of health interventions when making decisions on allocation of resources or implementation of policies and interventions. As a result, QALYs have been accepted as the "gold-standard" measurement of health-related utility in economic evaluations of healthcare interventions (Folland, Goodman, \& Stano, 2001).

\section{Cost-Effectiveness of Biopsychosocial Pain Interventions}

The decade following the published recommendations of the International Panel on Cost-Effectiveness in Health and Medicine has witnessed a rapid increase in CEA- 
and CUA-based economic evaluations of non-malignant pain and disability interventions in general (van der Roer, Boos, \& van Tulder, 2006; Vetter, 2007). Although the majority of CEA and CUA evaluations on non-malignant pain and disability focus on interventions for spinal disorders (e.g., Hatten, Gatchel, Polatin, \& Stowell, 2006; Rivero-Arias et al., 2005; Rivero-Arias, Gray, Frost, Lamb, \& StewartBrown, 2006), economic evaluations on other related fields of medical research have also been reported, including biopyschosocial treatments for temporomandibular disorders (Stowell, Gatchel, \& Wildenstein, 2007), workplace interventions for psychiatric patients (van Oostrom et al., 2008), and acute and subacute pain interventions in primary or secondary care facilities (Gatchel et al., 2003; Karjalainen et al., 2004).

Consistent with the trend of improved clinical outcomes when pain is addressed and treated based on the biopsychosocial perspective, economic evaluations have indicated that interdisciplinary and multidisciplinary pain and disability management programs are significantly more cost-effective than single-discipline treatment modalities and even surgery. A recent randomized controlled trial (RCT) comparing two types of single modality physiotherapy interventions to functional restoration for chronic low back pain reported superior cost-effectiveness of functional restoration at 18-months post-treatment, despite only marginal differences in clinical outcomes (Critchley, Ratcliffe, Noonan, Jones, \& Hurley, 2007). However, despite this, the functional restoration group was associated with significantly lower healthcare utilization at 18-months follow-up. Based on the United Kingdom's National Health 
Service's standard cost-effectiveness threshold of $£ 30,000$ per QALY or less, functional restoration had a $65 \%$ or greater probability of being more cost-effective compared to outpatient physiotherapy $(35 \%)$ and spinal stabilization classes $(<10 \%)$.

Similar results on cost-effectiveness were reported when a biopsychosocial postoperative rehabilitation intervention was utilized following lumbar fusion surgery, relative to standard post-operative care (Sogaard, Bunger, Laurberg, \& Christensen, 2008). The interdisciplinary rehabilitation group had a significantly greater, and constant, probability of being cost-effective in terms of improved pain and disability scores compared to the usual-care group. Additionally, slightly higher return-to-work rates at 2-years post-surgery were reported for the interdisciplinary rehabilitation group, relative to the usual-care group ( $60 \%$ vs. $50 \%$ ). These findings of cost-effectiveness in favor of biopsychosocial-based post-operative pain management are also robust across different types of surgeries in locations other than the low back, such as ENT surgery, abdominal surgery, plastic surgery, and gynecology-related surgery (Stadler, Schlander, Braeckman, Nguyen, \& Boogaerts, 2004). Furthermore, economic evaluations have also provided some guidance on the cost-effectiveness of surgery as a first-line intervention for chronic pain conditions. In an RCT of chronic low back pain patients comparing fusion surgery versus rehabilitation based on cognitive-behavioral therapy, the results indicated that fusion surgery is not a cost-effective first-line intervention for chronic low back pain, at approximately $£ 49,000$ per QALY gained (Rivero-Arias et al., 2005). Additionally, clinical outcomes at 2-years post-treatment indicated no significant differences between the surgery and rehabilitation groups. 
Cost-effectiveness of biopsychosocial interventions is not only limited to chronic pain conditions. In an evaluation of a biopsychosocial-based early intervention for acute low back pain patients who were at high-risk for developing chronic pain, Gatchel et al. (2003), demonstrated significantly greater cost-savings for the early intervention group relative to high-risk patients who did not receive early intervention. Additionally, the early intervention patients also reported significantly better clinical outcomes at one-year post-treatment, as well as greater return-to-work rates and reduced healthcare utilization. Similar results were reported from a study comparing intensive early intervention for subacute low back pain versus non-intensive usual care, with significantly reduced costs and higher return-to-work rates at two-years post-treatment for the early intervention group, despite no significant differences in self-reported pain and disability scores (Karjalainen et al., 2004).

As the studies reviewed above indicate, comprehensive pain and disability management programs based on the biopsychosocial perspective have been demonstrated to be highly cost-effective. In addition, the American Pain Society Task Force on Comprehensive Pain Rehabilitation has conducted a systematic review of the treatment and cost-effectiveness of biopsychosocial interventions for management of chronic non-malignant pain (Gatchel \& Okifuji, 2006). This review article estimated that, in the United States, biopsychosocial interventions for chronic pain and disability results in lifetime savings of $\$ 356,288$ per person. This is the lower limit of lifetime savings, which include savings of $\$ 83,678$ per person in lifetime disability payments, and savings of between $\$ 272,610$ - $\$ 423,279$ in lifetime healthcare costs (Gatchel \& 
Okifuji, 2006). The review also notes that factoring in other indirect costs, such as lost productivity, would result in substantially higher lifetime savings per person. Given that both treatment- and cost-effectiveness of biopsychosocial interventions for chronic pain have been firmly established in the literature, it is timely to investigate factors within a chronic pain patient population that, when identified, may play a role in further improving both treatment outcomes and cost-effectiveness of these established interventions.

\section{Early versus Delayed Rehabilitation}

\section{The Phase Model of Disability}

Krause and Ragland (1994) proposed the Phase Model of Disability, consisting of 8 phases of disability that correspond approximately to Gatchel's $(1991 ; 1996) 3$ Stage Model of Pain. Originally developed to explain occupational disability due to low back pain, the model is now gaining acceptance for occupational injuries in general (Franche, Frank, \& Krause, 2005; Franche \& Krause, 2003).The first five phases correspond to the acute and sub-acute stages of temporary disability resulting from occupational injuries. Of specific interest are phases 6,7 , and 8 , that were delineated as the chronic stages of disability and corresponded, respectively, to the following durations of disability: $3-6$ months; $6-18$ months; and greater than 18 months.

Patients falling under Phase 6 of the model are classified as requiring a change in case management and underscore the importance of intensive interdisciplinary treatment modalities in managing disability. Phase 7 of the model denotes a greater role played by psychosocial factors in maintaining disability, and that any treatment modalities at this 
point will require addressing psychosocial and socioeconomic barriers to recovery. Finally, Phase 8 is defined as the stage of permanent disability. Patients within this final stage, at greater than 18 months of disability, were suggested to have reached a terminal end-point with their disability, with a further implication that successful rehabilitation would be unlikely.

To test the disability model's assertion that patients beyond 18 months of disability would respond poorly to rehabilitation, Jordan, Mayer and Gatchel (1998) compared a cohort of short-term disability patients ( $4-8$ months duration) against two groups of patients with long-term disability (18 - 23 months and greater than 24 months), all of whom received functional restoration for work-related spinal injuries between years 1990 and 1993 . The results indicated statistically significant reductions in one-year work-related outcomes for the long-term disability groups. However, the magnitudes of outcome rates for the long-term disability were relatively comparable to the short-term disability group. For example, the long-term disability groups of 18-23 months and greater-than-24 months had return-to-work rates of $80 \%$ and $75 \%$ respectively, relative to a $93 \%$ return-to-work rate in the short-term disability group. Similar differences in magnitudes were observed for work retention at one-year postrehabilitation, with the long-term disability groups having retained work at $72 \%$ and $66 \%$, relative to the short-term disability group work retention rate of $80 \%$. Although treatment-effectiveness for early intensive rehabilitation is definitive, the results in that study indicated that patients with extremely long durations of disability could still be rehabilitated with respectable success rates after completing a course of intensive, 
interdisciplinary rehabilitation. However, the cost-effectiveness of a functional restoration program for patients with extended disability are not clear, especially given that the outcome magnitudes, although respectable, were still significantly lower than the early rehabilitation group. Likewise, the cost-effectiveness of functional restoration for patients in the early stages of chronic disability, relative to patients with longer disability durations, is as yet unknown.

\section{Cost Analysis of Disability Duration}

To date, there are only two published studies documenting the costs associated with length of disability within a musculoskeletal population, and neither one of them is a full economic analysis linking costs to outcomes. In a study on the effects of duration of disability on costs within a workers' compensation population for low back injuries, it was reported that $10 \%$ of patients with the costliest claims accounted for $86 \%$ of the total cost during the year 1992 (Hashemi, Webster, Clancy, \& Volinn, 1997). Additionally, approximately $75 \%$ of the total costs were due to a small minority of patients $(7 \%)$ who were disabled for more than a year. Similar results were obtained in an analysis of costs for work-related upper-extremity injuries, with patients disabled for more than one year (7\%) being responsible for approximately $60 \%$ of the total costs (Hashemi, Webster, Clancy, \& Courtney, 1998). To give context to these figures, disability compensation expenditures during this time period were estimated to be upwards of $\$ 43$ billion per year (Frymoyer \& Durett, 1997). Given that this small minority of patients with extended disability account for the majority of healthcare costs, it is timely to enumerate the cost-savings of early intervention for CDOMDs. 
Therefore, the proposed study provides an opportunity to contribute to an area of research that has insufficient data on the link between costs and outcomes as a function of disability duration.

\section{The Present Study}

\section{Overview}

The present study involves a cost-effectiveness analysis of the functional restoration approach to rehabilitation of patients suffering from chronic disabling occupational musculoskeletal disorders (CDOMD). Building upon the published literature on the treatment and cost-effectiveness of a biopsychosocial approach to pain management, this study was designed to determine the cost-effectiveness of early versus delayed rehabilitation of CDOMDs. The design of the cohort was modeled after disability duration ranges suggested by Krause and Ragland (1994), with some modifications based on Jordan, Mayer and Gatchel (1998). Specifically, the study was based on a consecutive cohort of patients who received functional restoration for CDOMDs. The cohort was divided into three groups based on duration of disability. The Early Rehabilitation (ER) group consisted of all patients who had a length of disability of between $4-8$ months. An Intermediate Duration (ID) group consisted of all patients who had a length of disability of between $9-18$ months. Finally, the Delayed Rehabilitation (DR) group consisted of all patients who had a length of disability greater than 18 months.

The groups were matched on several variables that are known to have an impact on outcomes following this treatment modality, specifically, age, gender, and injured 
musculoskeletal regions. Additionally, to ensure a complete match along demographic dimensions as well as to balance any potential socioeconomic confounds among the groups, ethnicity was also included as a matching criterion alongside age and gender. The cohort was evaluated on several pre- and post-rehabilitation factors, including psychopathology, perceived disability and function, and psychosocial well-being. Additionally, the groups were also compared on objective socioeconomic outcomes prospectively collected at one-year post-rehabilitation. Costs associated with the functional restoration program and relevant one-year costs associated with healthcare utilization were evaluated in a full economic evaluation, in order to determine the costeffectiveness of early versus delayed rehabilitation. The methodology of economic evaluation for this study was implemented based on guidelines published by the International Panel on Cost-Effectiveness in Health and Medicine (Gold, Siegel, Russell, \& Weinstein, 1996), and utilized both the CEA and CUA approaches of economic evaluation. Finally, data on estimated costs accrued over the duration of disability were presented, in order to provide an estimate for the overall cost-of-illness in a CDOMD cohort, as a function of disability duration.

\section{Specific Aims of the Study}

The major goal of this study was to document the cost-effectiveness of early intervention, utilizing functional restoration, for chronic musculoskeletal disorders within the Workers' Compensation setting. To aid in the meaningful interpretation of the results, several specific aims were formulated. These specific aims are: 
1. To demonstrate the consequences of extended durations of disability, specifically on the psychosocial characteristics of patients during time of admission to the program.

2. To reinforce previous findings on the treatment-effectiveness of early rehabilitation by evaluating post-treatment psychosocial outcomes, as well as one-year objective socioeconomic outcomes.

3. To determine the incremental cost-effectiveness of early rehabilitation versus delayed rehabilitation, as well as to enumerate the probability of the treatment strategy (timeliness of rehabilitation) being cost-effective.

4. To determine or estimate all costs accrued over the duration of disability, including medical costs, disability benefits, productivity losses, as well as the costs associated with the functional restoration program and one-year posttreatment utilization of healthcare resources. In total, these individual cost components provide an estimate of the overall cost-of-illness associated with disability due to work-related injuries.

\section{Hypotheses}

1. The ER group will demonstrate significantly lower psychopathology and significantly more positive responses on functional and psychosocial measures at pre-rehabilitation, compared to the ID and DR groups.

2. The ER group will be associated with significantly more positive responses on functional and psychosocial measures at post-rehabilitation, compared to the ID and DR groups. 
3. The ER group will be associated with significantly better rates on objective outcomes at one-year post-rehabilitation, compared to the ID and DR groups.

4. Early rehabilitation will demonstrate greater cost-effectiveness at one-year postrehabilitation, based on both objective outcome measures as well as on healthrelated quality of life measures. 


\section{CHAPTER 2}

\section{METHODS}

\section{Patients}

A consecutive cohort of 2,491 patients were initially identified as being eligible this study, based on diagnoses for chronic disabling occupational musculoskeletal disorders (CDOMD). All patients were admitted to a functional restoration program at the Productive Rehabilitation Institute of Dallas for Ergonomics (PRIDE), between the years of 1998 and 2005. The Workers' Compensation jurisdiction for this cohort is predominantly from the Texas Workers' Compensation system (90\%), with the remaining patients from other State Workers' Compensation jurisdictions, Federal disability compensation systems, Texas Non-Subscribers, as well as private insurance and personal injury policies. Program participation criteria include: 1) four or more months elapsed since a work-related injury; 2) acute conservative care and/or secondary care failed to improve symptoms sufficient to allow full return to work; 3) surgery had not produced relief, resolution or simply was not an option; 4) severe pain and functional limitations remained; and 5) ability to communicate in English, Spanish or other translatable languages. In addition to the general program inclusion criteria, this initial cohort was subject to several exclusion criteria to provide a working cohort for the study. The following exclusion criteria apply: 
1. Exclusion of patients covered by the Federal Employees Liability Act (FELA) for railroad workers. This sub-sample of patients, $N=24$ and corresponding to $1 \%$ of the total cohort, were excluded due to their nature as extreme outliers in the disability compensation system. Patients covered by FELA are a unique sub-sample that are noted for receiving significantly higher amounts of disability compensation or settlements, relative to preinjury wages, and are noted for significantly poorer outcomes relevant to the Workers' Compensation setting (such as return-to-work). Furthermore, due to the very small prevalence of FELA cases available in this cohort, analyzing them as a separate sub-group will not be feasible.

2. Exclusion of patients covered by private insurance. This small sub-sample of patients, totaling $N=13$ at $0.6 \%$ of the total cohort, did not receive the full course of interdisciplinary functional restoration and were only treated on an out-patient basis approximately 2 or 3 times per week, mainly with physical therapy.

3. Program non-completers $(N=589,24 \%$ of total cohort $)$ were also excluded from the study. This exclusion criterion is necessary as there is minimal documentation of program non-completers in patient research folders, preventing collection of any data relevant to cost estimation. Additionally, one-year outcomes on program completers are only obtained at a contact rate of approximately $50 \%$. Finally, no details on potential alternative treatment 
modalities undergone by non-completers during the usual one-year program outcome period are available, and would therefore prevent accurate quantification of any post-program costs and their impact on the limited amount of one-year outcomes available on non-completers. It should be noted that, prior to constructing the matched cohort, the completion rate across the disability duration groups were analyzed to identify any effect for differential drop-out rates across the groups.

\section{Cohort Structure}

\section{Overview}

A matched cohort design was utilized for this study. The total cohort was divided into three groups based on length of disability. The division of the groups was based on the chronic disability phases of the developmental disability model proposed by Krause and Ragland (1994), as well the cohort structure utilized by Jordan, Mayer and Gatchel (1998) to investigate the effect of extended disability on outcomes following functional restoration. The three groups in this study correspond to Early Rehabilitation, Intermediate Duration, and Delayed Rehabilitation.

The Early Rehabilitation (ER) group corresponds to Phase 6 of Krause and Ragland's (1994) model, which involves durations of between $3-6$ months of disability. However, as similarly defined by Jordan, Mayer and Gatchel (1998), the early rehabilitation group was modified to include all patients disabled for up to 8 months in total. Given the program inclusion criteria corresponding to disability duration in the previous section, the early rehabilitation group included all patients with 
disability durations of between $4-8$ months. The rationale for extending the duration up to 8 months is to take into account the time needed for recuperation from any surgery, as well as administrative time needed for insurance carrier approval for the patient's enrollment in the functional restoration program. The Intermediate Duration (ID) group corresponds approximately to Phase 7 of Krause and Ragland's (1994) model, with duration of disability ranging between $9-18$ months. Finally, the third group classified as Delayed Rehabilitation (DR) corresponds to Phase 8 of Krause and Ragland's (1994) model with a duration of disability of more than 18 months.

\section{Matching Criteria}

The three groups were matched on several variables to facilitate accurate comparison among the groups on rehabilitation outcomes and the results of the costeffectiveness component of the study. Variables selected to be matched for among the groups included gender, age, ethnicity, and injured region of the body. The research literature on disability rehabilitation indicate that these factors are associated, both directly and indirectly, with poorer outcomes at post-treatment, such as lower return-towork rates, lower likelihood of retaining work, and increased healthcare utilization during one- and two-years follow-up (DeBerard, Masters, Colledge, Schleusener, \& Schlegel, 2001; Dersh et al., 2008; Gatchel, Mayer, Kidner, \& McGeary, 2005; Maghout-Juratli, Franklin, Mirza, Wickizer, \& Fulton-Kehoe, 2006; Mannion \& Elfering, 2006; Mayer, Gatchel, \& Evans, 2001; McGeary, Mayer, Gatchel, Anagnostis, \& Proctor, 2003; Theodore, 2007). 
Following the application of the exclusion criteria described above, a cohort of $N=1,865$ program completers remained available for the study. The patients were approximately equally distributed among the three groups with $N=683(37 \%), N=608$ (33\%), and $N=574$ (31\%) corresponding to the ER, ID, and DR groups, respectively. The ER group was used as the index group based upon which the ID and DR groups were matched. The method of matching used was category matching (Dicker, 2002; Rothman, Greenland, \& Lash, 2008), to ensure that all groups had a similar distribution on age, gender, injured musculoskeletal regions, and ethnicity. To facilitate this method of matching, the age variable was categorized into 5 categories corresponding to $\leq 30$ years, $31-40$ years, $41-50$ years, $51-60$ years, and $>60$ years. Overall, $N=138$ patients in the ER group could not be matched to the ID and DR groups, leaving $N=$ 545 eligible patients in the ER group. For the ID group, a total of $N=472$ patients were matched to the ER group based on similar distributions on the four matching variables. Similarly, a total of $N=446$ patients from the DR group were matched to the ER group.

Within these two matched sets, $N=99$ patients in the ID group and $N=73$ patients in the DR group did not overlap on matched variables (total of $N=172$ ), although both groups were individually matched to the index group. After excluding this $N=172$ sub-sample of non-overlapping individuals, the final matched cohort consisted of $N=1,119$ patients, with $N=373$ patients in each of the three groups. All three groups had exactly similar distributions on the three demographic variables, as well as the injured musculoskeletal regions of the body. It should be noted that this method of group matching, when utilized in cohort studies, reliably eliminates the 
confounding effects of the matched variables among the groups and on the evaluated outcomes (Aschengrau \& Seage, 2003).

\section{Measures}

\section{Demographic Data}

Demographic data was obtained from patient records in the PRIDE Research Foundation's electronic database, and included the following variables: age; gender; race; injured musculoskeletal regions; marital status; level of education; and, length of disability.

Pre-Rehabilitation Medical Data

Pre-rehabilitation medical data was obtained from patient records in the PRIDE Research Foundation's electronic database as well as patient charts systematically assembled for research purposes. These data include: the number and type of prerehabilitation surgeries; diagnostic procedures; injection therapeutics; and the amount of pre-rehabilitation healthcare utilization of non-surgical treatment modalities (primary and secondary care, chiropractic manipulation therapy, and pain management). Pre-Rehabilitation Occupational and Workers' Compensation Characteristics

Pre-rehabilitation occupational data were obtained from patient records and case management data available in the PRIDE Research Foundation's electronic database. These data include the following variables: type of occupation; physical demands of occupation; pre-injury weekly net wages; pre-rehabilitation occupational status; availability of original job; months of Temporary Total Disability (TTD); Workers' 
Compensation jurisdiction; Workers' Compensation case settlement status, percentage of patients on Social Security Disability Insurance (SSDI); and, attorney retention. Psychosocial Measures and Psychiatric Diagnosis

Psychosocial measures were collected both at a pre-rehabilitation intake interview and at the completion of the rehabilitation program. Validated clinician-rated and self-report questionnaires used in this study include: the Hamilton Depression Rating Scale (HAM-D)(Hamilton, 1960); the Million Visual Analog Scale (MVAS)(Anagnostis, Mayer, Gatchel, \& Proctor, 2003) for measuring perceived disability; the Medical Outcomes Short-Form 36 Health Survey (SF-36)(Ware, Snow, Kosinski, \& Gandek, 1993); and a visual analog scale measuring pain intensity (McGeary, Mayer, \& Gatchel, 2006).

Post-injury Axis I psychiatric diagnoses based on the Diagnostic and Statistical Manual of Mental Disorders, Fourth Edition - Text Revision (DSM-IV TR)(American Psychiatric Association, 2000), were established at the pre-rehabilitation intake interview using the Structured Clinical Interview for the DSM-IV (SCID-I)(First, Spitzer, Gibbon, \& Williams, 1997a). Major post-injury DSM-IV Axis I diagnoses prevalent in the CDOMD patient population were investigated in this study, including Major Depressive Disorder, Anxiety Disorders, Post-Traumatic Stress Disorder, and Substance Use Disorders (abuse and dependence diagnoses for alcohol, opioids, and non-opioid drugs). Additionally, DSM-IV Axis II diagnoses for Personality Disorders (PD) were also established during the pre-rehabilitation intake interview, using the Structured Clinical Interview for DSM-IV Personality Disorders (SCID-II)(First, 
Spitzer, Gibbon, \& Williams, 1997b). Major DSM-IV Axis II disorders prevalent in the CDOMD population were also investigated in this study, and included Paranoid PD, Borderline PD, Histrionic PD, and Obsessive-Compulsive PD. One-Year Socioeconomic Outcomes

One-year post-rehabilitation socioeconomic outcomes were collected using a structured telephone interview (Mayer, Prescott, \& Gatchel, 2000). These socioeconomic outcomes used in this study included: return-to-work (RTW); work retention at one-year; percentage seeking treatment from new healthcare provider; mean visits to a new healthcare provider; new surgeries to original area of injury; new compensable injuries; and, Workers' Compensation case settlement status. In addition to the conventional set of socioeconomic outcomes relevant to the Workers' Compensation setting, an additional one-year socioeconomic outcome to be included in this study is disability income source at one-year post-rehabilitation. Table A.1 operationally defines these objective socioeconomic outcomes.

\section{Cost Estimation}

\section{Overview}

Cost data used for this study can be broadly divided into four categories: Medical Costs; Lost Productivity and Disability Benefits; Functional Restoration Program Costs; and One-Year Post-Rehabilitation Costs. Table A.2 summarizes a cost inventory for all costs used in the study. Costs incurred prior to the functional restoration program serve as benchmark cost descriptors for comparing the three groups in the cohort. Additionally, these pre-rehabilitation costs are also used to quantify the 
overall cost-of-illness in this CDOMD cohort, specifically documenting the magnitude of costs associated with extended periods of disability. Program costs and one-year post-rehabilitation costs will form the basis of the full economic evaluation, and were utilized as the cost component within the analytical timeframe of the Cost-Effectiveness Analysis.

\section{Medical Costs}

Although general medical procedures in a patient's history are documented in PRIDE Research Foundation's electronic database and patient charts, detailed data on specific modalities, amount of utilization needed for costing, and costs associated with these procedures are either unavailable or not reliably recorded. Therefore, these costs have to be estimated from sources external to, and independent of, the present functional restoration program. Estimates for medical costs were obtained from a $10 \%$ random sample of unique claims with valid medical cost data from a comprehensive claims database managed by the Ohio Bureau of Workers' Compensation (OBWC). Medical costs included all associated costs for a work-related injury, including surgeries, diagnostics, injection therapeutics, hospital costs, primary and secondary care modalities, chiropractic treatment, pain management, psychological services, case management services, pharmaceuticals, and costs associated with physicians' consultation and evaluation and management services.

The $10 \%$ random sample from OBWC's claims database consisted of $N=4,726$ unique claims between years 1998 and 2005, for work-related injuries in Ohio State's Workers' Compensation jurisdiction. The acquisition of the dataset was based on a 
match of International Statistical Classification of Diseases and Related Health Problems, Ninth Edition (ICD-9) diagnosis codes for musculoskeletal disorders that are prevalent in the PRIDE patient population. It should be noted that the distribution of injured musculoskeletal regions in this OBWC cohort were consistent with recently published estimates on national trends for the prevalence of these work-related musculoskeletal injuries (U.S. Bureau of Labor Statistics, 2007).

Further refining of a comparable subset from this data was undertaken to facilitate accurate estimates of medical costs for this study. This was done by using the category matching method to ensure that both the PRIDE and OBWC cohorts were comparable for the purpose of extracting estimates of medical costs. While an attempt to match based on all relevant variables was initially undertaken (i.e., gender, age, length of disability, injured musculoskeletal regions, and type of surgery), the substantial lack of overlap between the two cohorts resulted in very small sample sizes of around $50 \%$ or less of the total PRIDE cohort. To maximize the greatest number of patients in order to provide reliable estimates of medical costs, the final criteria for matching was based on the distribution of injured musculoskeletal regions and type of surgery. Finally, the medical cost estimates were screened to identify and remove any abnormalities in the data obtained from OBWC. These details are elaborated upon in the results section.

\section{Lost Productivity and Disability Benefits}

The costs associated with lost productivity due to disability were estimated using the Human-Capital method of costing productivity (Hodgson, 1994; Rice, 
Hodgson, \& Epstein, 1985). Specifically, this method entails multiplying the average weekly wage of the patient by the duration of time absent from work. To estimate productivity losses in the PRIDE cohort, the pre-injury average weekly wage was converted to an average monthly wage, and then multiplied by the duration of Temporary Total Disability (TTD) in months. For the costs associated with disability benefits, estimates were obtained by applying jurisdictional rules relevant to the individual Workers' Compensation system that patients were covered by.

Texas Workers' Compensation. The TTD benefit, a type of wage-loss compensation, associated with the Texas Workers' Compensation (WC) system is referred to as Temporary Income Benefits (TIBS). The specific methodology used in this study for estimating the weekly TIBS rate is detailed in Appendix C, based on a publication by the Texas Department of Insurance's Workers' Compensation Division. In general, the weekly TIBS rate is equivalent to $70 \%$ of the average weekly wage, but not exceeding statutory ceiling limits based on the State average weekly wage for the given fiscal year that the injury occurred. However, for workers' earning less than $\$ 8.50$ per hour, the first 26 weeks of TIBS is computed at $75 \%$ of the average weekly wage, and reverts to $70 \%$ following this initial period, in both cases to not exceed the statutory ceiling limit. The duration of TIBS is for a maximum of 104 weeks, or until the injured worker is defined to have reached maximum medical improvement (whichever comes first).

Federal Employees' Compensation Act (FECA). This is the jurisdiction that covers all the injured workers in the study cohort that are Federal workers. Disability 
benefits for injured workers' covered under FECA were computed based on specific guidelines published by the United States Department of Labor (U.S. Department of Labor, 1999). In general, weekly FECA benefits are computed at $66.7 \%$ of the average pre-injury weekly wage for injured workers with no dependents, and $75 \%$ of the average pre-injury weekly wage if the injured worker is married or has one or more dependents. However, injured workers' whose injury involved a traumatic injury (as opposed to repetitive motion/stress/strains) maintain a continuation of pay at full wage rate for the first 45 days of the disability. For the injured workers in the PRIDE cohort that were under the jurisdiction of FECA, the presence of dependents was unable to be determined. However, those who were identified as being married had their FECA benefit rate computed at the mandated compensation rate. The weekly compensation rate under FECA, after being converted to a monthly payment, was then multiplied by the duration of months in TTD. Those patients identified, through the PRIDE research folders, as having a traumatic injury also had 45 days of continuation of pay accounted for in the total amount of disability benefit accrued.

Texas Non-Subscribers. This category of injured workers corresponds to those whose employers opted out of the Texas Workers' Compensation system regulated by the Texas Department of Insurance. The TTD disability benefit rate for NonSubscribers were computed based on benefits rates reported by The Comp Solutions Network, Inc (http://www.compsolutionsnetwork.com/products.htm). The TTD benefits rate for Non-Subscribers corresponds to $75 \%$ of the average weekly wage, up to a maximum of $\$ 700$ per week. The duration of benefits is up to 156 weeks. For the 
injured workers in the PRIDE cohort who were classified as Non-Subscribers, total benefits accrued during the period of disability was computed by multiplying the monthly compensation rate for TTD benefits by the duration of TTD in months.

Other State Workers' Compensation. Injured workers' represented by State Workers' Compensation outside Texas included those from California, Colorado, Florida, Georgia, Indiana, Iowa, Montana, Oklahoma, Tennessee, Washington, and Wisconsin. Benefits rates for TTD were computed based on the individual jurisdiction rules mandated by each State Workers' Compensation System. The benefits rate for TTD corresponded to $66.7 \%$ of the average weekly wage, with the exception of Oklahoma (70\%) and Iowa (85.7\%). Specific rules for each State were obtained from an online directory of Workers' Compensation jurisdictions, maintained by the School of Labor \& Industrial Relations at Michigan State University (http://www.lir.msu.edu/wcc/wcid/wc state.php).

Impairment Income Benefits (IIBS). This benefit amount is awarded for permanent impairment due to a work-related injury. It should be noted that all patients receiving IIBS in the cohort for this study were from the Texas Workers' Compensation system. Therefore, the specific rules mandated by the Texas Department of Insurance were used in computing the IIBS rate and are detailed in Appendix D. In general, IIBS payments begin after an injured worker receives an impairment rating, either at the time of determination for maximum medical improvement or after 104 weeks has elapsed and TIBS payments are stopped. Impairment ratings are a percentage that documents the degree of permanent damage to the body as a whole, and are assigned based on 
guidelines published in the American Medical Association's Guides to the Evaluation of Permanent Impairment (American Medical Association, 2008). Every percentage point of impairment entitles the injured worker to 3 weeks of IIBS payments. The actual IIBS rate, based on guidelines for Texas, corresponds to $70 \%$ of the injured workers' pre-injury weekly wage, and not to exceed a statutory ceiling limit of $70 \%$ of the State average weekly wage. For the PRIDE cohort, patients receiving IIBS payments had their IIBS rate computed according to the method mandated by the Texas Department of Insurance. Following this, each patient's impairment rating was identified from the PRIDE research folders and the IIBS rate was multiplied by the number of weeks that were eligible for IIBS payments (i.e., 3 weeks per $1 \%$ of impairment rating).

\section{Program Costs}

Cost data directly associated with the PRIDE functional restoration program include treatment billing hours for the functional restoration program, the cost of doctor visits both at initial assessment and during the one-year post-rehabilitation period, as well as any injection therapeutics that patients received as part of the preparation for the intensive phases of the functional restoration program. All these costs were sourced from PRIDE Research Foundation's electronic database. Any medications prescribed upon admission or at discharge were sourced from patient charts compiled in the PRIDE research folders. The costs for medication were estimated using unit costs of each medication as provided by Epocrates, Inc (http://www.epocrates.com), a private corporation that maintains a publicly accessible electronic database of pharmaceutical costs. Where multiple dosage packs were available, a conservative estimate was 
obtained by using the unit cost of the smallest dosage pack. For certain brand name pharmaceuticals that are currently pulled off the market (e.g., Vioxx), average unit cost for that specific brand-name pharmaceutical was extracted from the OBWC billing database.

\section{One-Year Post-Rehabilitation Costs}

Costs incurred during the one-year post-rehabilitation period included any new surgeries for the originally injured compensable region and the cost of visits to new healthcare providers. While the OBWC dataset served as the main source of estimates for relevant medical costs, the inherent structure of this database evidenced some limitations in being able to provide a combined estimate of specific surgical costs (i.e., the surgeon's cost) with all surgery-related ancillary costs such as diagnostics, any presurgical interventions, post-surgical interventions, and hospital stays. This limitation has an adverse impact in being able to estimate one-year post-rehabilitation surgery costs among the PRIDE patients.

Given the above, an additional source for estimating the cost of surgeries was utilized for surgeries identified during the one-year post-rehabilitation period. These cost estimates were sourced from the Centers for Medicare and Medicaid Services (CMS) program for Health Care Consumer Initiatives (http://www.cms.hhs.gov/HealthCareConInit/02_Hospital.asp). This report by the CMS provides a general estimate of costs by 31 major categories of surgeries, of which 10 were identified for specific musculoskeletal surgeries. The cost estimate for each category of surgery includes ancillary costs such as hospital stays, diagnostics, and 
anesthesia, in addition to the surgeon's cost. Table A.3 details the estimated costs per category of surgery, in 2005 dollars.

Visits to new healthcare providers during the post-rehabilitation year were estimated using a unit cost per office-based physician visit provided by the Agency for Healthcare and Research Quality (AHRQ). The unit cost per visit was estimated to be \$72, based in nominal dollars for year 2004 (approximately \$75, when adjusted to 2005 dollars) (Machlin \& Carper, 2007).

\section{Procedure}

All patients were enrolled in a functional restoration program at PRIDE, and consented to the collection of data for the purposes of rehabilitation management, workers' compensation documentation, and research. Functional restoration is an intensive, medically supervised interdisciplinary program, combining quantitatively directed exercise progression with a multimodal disability management approach, incorporating psychological and case management techniques (Hazard, 1995; Hazard et al., 1989; Mayer \& Gatchel, 1988). Developed in 1983, this rehabilitation program is a variant of chronic pain management based on the biopsychosocial model of pain and disability, and is specifically intended for rehabilitation of compensation injuries (Mayer et al., 1985). The efficacy of functional restoration for CDOMD, as well as the objective outcomes for treatment monitoring, have been extensively reviewed in the literature (Bendix et al., 1996; Hildebrandt, Pfingsten, Saur, \& Jansen, 1997; Jousset et al., 2004; Mayer et al., 1998; Mayer et al., 1985; Mayer et al., 1987; Rainville, Kim, \& Katz, 2007; Wright, Mayer, \& Gatchel, 1999). 


\section{Initial Evaluation}

All patients within this cohort received an initial multidisciplinary evaluation consisting of medical history, physical examination, quantitative functional evaluation, psychological intake interview, medical case management, and a disability assessment interview. These initial evaluations are required components that help guide the duration and intensity of the rehabilitation regimen by identifying physical and psychosocial limitations of each patient. As such, the rehabilitation process can be tailored towards being maximally efficient for each individual patient. Demographic data and pre-rehabilitation medical, occupational, and psychosocial measures on all patients were collected during this initial evaluation.

Medical history. These evaluations were conducted via access to patient health records from patients' primary care physician. Information gathered from this evaluation included the nature of injury, date of injury, types of surgery (if any), date of surgery (if any), treatment modalities and levels of care previously administered, as well as prior and current medication regimen.

Physical and functional capacity evaluations. These evaluations were used to assess a patient's “weak link”. For chronic pain patients who are suffering from physical deconditioning, the weak link is the injured area of the body that suffers from limited mobility, strength, and endurance compared to other regions of the body, and is usually the source of the patient's pain and disability. While physical capacity evaluations measured mobility, strength, and endurance at the weak link, functional capacity evaluations assessed patients' ability in performing functional tasks, such as 
lifting, squatting, climbing, and bending. Reliable measurement apparatus were utilized in these evaluations, including inclinometers for measuring mobility, as well as isometric, isokinetic, and isoinertial devices for measuring strength, endurance, and functional capacity.

Psychosocial assessment. The accurate identification of psychosocial barriers to recovery and risk factors for poorer treatment outcomes, are a central component of functional restoration. All patients completed a battery of screening tests for perceived disability, symptom magnification, somatization, depressive symptoms, and general health status. These psychosocial assessments included those selected for the present study, such as the MVAS, the SF-36, and the visual analog scale for pain intensity. Additionally, trained clinicians administered the HAM-D, SCID-I, and SCID-II during this period to document depressive symptoms as well as Axis I and Axis II psychopathology based on the DSM-IV.

Case management and disability assessment interview. All patients received a comprehensive interview with case managers in order to evaluate the nature of the workers' compensation claims, as well as any other compensation the patient may currently be receiving. In addition to the nature of compensation, this stage of the evaluation also determined if patients had any pending litigation associated with their injury, and if they had attorney representation. Additional information gathered during this interview process was also used to identify potential risk factors for poorer treatment adherence and outcomes, as well as any socioeconomic barriers to recovery. 
This included, for example, pre-injury occupational demands, level of job satisfaction prior to disability, relationship with employer, and pre-injury wage levels.

\section{Rehabilitation}

Phase 1. The rehabilitation stage of a functional restoration program is divided into three phases. The first phase of the program included narcotic detoxification, psychotropic medication management, and light aerobic and mobility training. Patients may be prescribed non-habituating anti-inflammatory medication at this phase if pain is exacerbated by the light exercises. The primary goal of the first phase is to "warm up" the patients for the intensive second phase of the rehabilitation. The light aerobic and mobility exercises also allow patients a gradual increase of activity at their deconditioned site of injury.

Phase 2. Once all the objectives of the first phase were satisfied, the program continued with an intensive second phase involving strength and endurance training under the direction of physical and occupational therapists. This stage of functional restoration has a two- to three-week duration, depending on the initial evaluations and the extent of patients' physical and psychosocial deconditioning. Initial stages of physical therapy involved focused rehabilitation of the weak link within a supervised environment, guided by pre-rehabilitation physical and functional capacity evaluations. Physical exercises were incrementally intensified over the duration of the second phase. Once optimal rehabilitation of strength, mobility, and endurance of the weak link was achieved, patients were then guided through occupational therapy which involved coordinating the weak link with other regions of the body in attempting functional tasks 
that simulate activities of daily living, as well as common tasks found in the workplace. Counseling and training in coping skills, pain and stress management, and maintaining the goal of returning to work were provided by psychologists and counselors throughout the duration of the second phase. The psychosocial interventions were also targeted towards addressing any fear-avoidance issues that patients may be exhibiting.

Phase 3. Upon completion of the intensive phase of the program, patients were provided with education geared towards maintaining program goals, instruction on home exercise regimens for fitness maintenance, additional counseling, and any necessary non-habituating psychotropic or anti-inflammatory medications. Case managers also provided occupational placement services, helped with any negotiations with patients' employers for temporary light or modified duty upon return to work, as well as in helping with workers' compensation case settlement issues. This final phase of the functional restoration program had a two- to three-week duration, and concluded with a post-program quantitative evaluation. This evaluation consisted of the various physical and a functional capacity evaluations, as well as the psychosocial battery of self-reports administered during the pre-rehabilitation evaluation.

One-year Follow-up Structured Interview

One-year follow-up structured interviews for gathering socioeconomic outcome data were conducted by interviewers independent of the rehabilitation team, and were unknown to the patients (Mayer, Prescott, \& Gatchel, 2000). Efforts were made to contact all patients by telephone, unless they appeared for the interview in person. Data were also gathered from additional sources, such as employers, insurance carriers, 
family members, and attorneys (if patients had representation). Multiple points of data collection ensure reliable outcomes, as well as partial data on outcomes if the patient could not be directly contacted. All one-year socioeconomic outcomes were collected during this structured interview. These outcomes have been consistently reported from this program in the past, and have been shown to be reliable discriminant indicators of patients who complete the program compared to those that refuse treatment or do not complete it (Mayer et al., 1985; Mayer et al., 1987; Proctor, Mayer, Theodore, \& Gatchel, 2005). In addition, direct contact rates ranging from $93 \%$ - 98\% have been consistently reported from this program (Dersh et al., 2008; Dersh et al., 2007; Gatchel, Mayer, \& Theodore, 2006; Jordan, Mayer, \& Gatchel, 1998; Mayer, Towns, Neblett, Theodore, \& Gatchel, 2008; McGeary, Mayer, \& Gatchel, 2006; Proctor, Mayer, Gatchel, \& McGeary, 2004; Proctor, Mayer, Theodore, \& Gatchel, 2005; Wright, Mayer, \& Gatchel, 1999). Finally, the reliability of one-year socioeconomic outcomes was also previously documented in evaluating outcomes obtained from a two-year follow-up interview reported from this same program (Mayer et al., 1987).

\section{Conventional Data Analysis}

Distributional Assumptions. Cost data are noted for having distributions that deviate substantially from normality. Conventional procedures for rectifying this issue range from using distribution medians, non-parametric statistical analyses, or conducting transformations on the data (e.g., logarithmic transformations). In the present study, initial evaluations of the cost data indicated severe heterogeneity of variances among the groups, in addition to extreme levels of positive skew. Although 
deviation from normality is not a substantial problem for parametric statistical analyses (Hays, 1994; Tabachnick \& Fidell, 2001), heterogeneity of variances can lead to statistical conclusion errors. Therefore, in order to maintain the assumption of homogeneity of variances, all cost data were subject to transformations using the natural logarithmic scale when variances significantly differed among the groups based on Levene's test for homogeneity of variances. These re-scaled variables were then utilized in the relevant parametric statistical analyses, and are reported alongside their untransformed means and medians. This transformation rule was similarly applied to all non-cost continuous variables as well, when significant deviations from the assumption were observed.

Univariate tests. Tests of association were conducted based on the Pearson chisquare $\left(\chi^{2}\right)$ test statistic for analyses of differences among the ER, ID, and DR groups on all categorical variables. One-way Analysis of Variance (ANOVA) was conducted for analyses of differences among the ER, ID, and DR groups on continuous variables. Both tests also included planned comparisons for linear trends across the disability duration groups. The significance criterion for all tests were set at $\alpha=.05$. Effect sizes for all significant $p$-values are reported: consisting of partial eta-squared $\left(\eta^{2}\right)$ for all continuous variables $(0.01=$ small, $0.09=$ medium, $0.25=\operatorname{large})$; and Cohen's $w$ for all categorical variables $(<0.3=$ small, $0.3-0.5$ medium, $>0.5=$ large $)$. When a significant effect was indicated for the omnibus tests, post-hoc tests were conducted to compare each of the late disability duration groups (ID and DR groups) against the ER group. Dunnett's test was utilized as the post-hoc test for continuous variables, utilizing 
the ER group as the reference group. Cohen's $d$ was reported for all significant post-hoc comparisons on continuous variables $(0.2=$ small, $0.5=$ medium, $0.8=$ large $)$. For categorical variables, a logistic regression analysis was conducted using disability duration groups as the predictor with ER as the reference group, and a Bonferroni correction that adjusted the critical value for significance to $\alpha=.025$. The odds-ratio and 95\% CI was reported for all significant post-hoc comparisons on categorical variables.

Multivariate tests. All post-rehabilitation psychosocial measures were analyzed using an Analysis of Covariance (ANCOVA), with the corresponding pre-rehabilitation psychosocial measure as a covariate. In addition, a sequential regression analysis was conducted for costs within the analytical timeframe to identify any significant costdrivers over and above any effect of disability duration. These included any prerehabilitation medical, occupational, and psychosocial measures that significantly differed among the three disability groups. Specifically, the variables analyzed as predictors included depressive symptoms at pre-rehabilitation (HAM-D), perceived disability at pre-rehabilitation (MVAS), Major Depressive Disorder, Alcohol Abuse Disorder, Opioid Dependence Disorder, Non-opioid Drug Dependence Disorder, prerehabilitation job availability, Workers' Compensation case closure at admission, attorney retention, and any pre-rehabilitation surgeries. These variables were included in the regression analysis as a separate block, after adjusting for the effect of disability duration. The significance criterion for all tests were set at $\alpha=.05$. Prior to running the regression analyses, all predictor correlation magnitudes were evaluated in a correlation 
matrix to ensure there was no redundancy among the predictors that may result in problems of multicollinearity.

Economic Analysis

Overview. A full economic analysis is presented and included both the CEA and CUA approach, utilizing methods recommended by the International Panel on CostEffectiveness in Health and Medicine (Gold, Siegel, Russell, \& Weinstein, 1996). As discussed earlier, both approaches differ only in terms of the outcomes utilized in the "effectiveness" component of the economic analysis. The analytical timeframe begins at the point of admission to the functional restoration program, and terminates at the point of data collection for the one-year outcomes.

Cost Component. The cost component of both the CEA and CUA approaches included the Program Costs as well as the One-Year Costs as described in a previous sub-section. Equations 1 through 3 describe the cost component of the economic evaluation:

- Program Costs $=$ Cost of Functional Restoration Treatment Hours + Cost of Doctor Visits + Cost of Therapeutic Injections + Cost of Medications (Eq. 1)

- One-Year Costs $=$ Cost of Surgeries (for original injury) + Cost of Visits to New Healthcare Providers (Eq. 2)

- Total Costs in Analytical Timeframe $=$ Program Costs + One-Year Costs (Eq. 3) 
It should be noted that costs incurred prior to the intervention cannot be utilized within the analytical timeframe of the economic evaluation, but can be reported as benchmark descriptors for the overall cost of illness.

Outcomes for CEA. Outcomes for the CEA portion of the economic evaluation utilized natural-unit outcomes relevant to the Workers' Compensation setting for CDOMDs. These included RTW and work retention at one-year. It should be noted that the other one-year outcomes such as the costs associated with healthcare utilization and new surgeries are already included in the cost component of the analysis.

Outcomes for CUA. Outcomes for the CUA component of the economic evaluation consisted of the SF-36 scores at pre- and post-rehabilitation. These SF-36 scores were converted to health-related utility scores based on the Quality of WellBeing (QWB) scale, by using a validated scale-level conversion algorithm developed during the Beaver Dam Health Outcomes Study (Fryback, Lawrence, Martin, Klein, \& Klein, 1997). Table A.4 summarizes the specific SF-36 scales and the regression weights used in this conversion algorithm. Further successful validation of this conversion algorithm across diverse clinical populations, including chronic pain patients, has been reviewed in the literature (Hollingworth et al., 2002; Pickard, Wang, Walton, \& Lee, 2005). The resulting utility scores represent changes in health-related quality of life (QoL) at post-treatment, a measure analogous to a QALY except that its duration is less than one-year post-rehabilitation.

Average Ratios. The Average Cost-Effectiveness Ratio (ACER) and the Average Cost-Utility Ratio (ACUR) are one set of the two major statistics in economic 
evaluations. The ACER and ACUR define the overall affordability for an intervention, given the outcome under consideration. For example, these average ratios indicate the cost per unit outcome of RTW, or the cost per unit gained in QoL. The average ratios were computed for each group, on each of the outcome measures utilized. The computation method for both the ACER and ACUR are identical, except the denominator for ACUR was based on the average unit of QoL gained per group whereas the ACER was based on the natural-unit outcome rates under consideration (e.g., RTW per group). The average ratio is defined by the equation 4:

$$
\text { - Average Ratio group }=\frac{\text { Average Cost }_{\text {group }}}{\text { Average Outcome }_{\text {group }}} \text { (Eq. 4) }
$$

Incremental Ratios. The Incremental Cost-Effectiveness Ratio (ICER) and the Incremental Cost-Utility Ratio (ICUR) are another set of major statistics in economic evaluations. The ICER and ICUR define the cost of one additional outcome of the intervention under consideration, relative to a comparator. For this study, these incremental ratios allow evaluation of early rehabilitation relative to intermediate duration and delayed rehabilitation. These were computed by initially sorting the groups by increasing effectiveness for a given outcome (e.g., lowest RTW rates to highest RTW rates). Following this, incremental ratios were computed for each group by comparing it to the group with the next best outcome (e.g., a group with RTW of $90 \%$ is compared to the next highest group, say $80 \% \mathrm{RTW}$, which in turn is compared to the final group that has the lowest outcome rate of, say, $70 \% \mathrm{RTW})$. Therefore, the group with poorer outcomes serves as the comparator to enable computation of the cost of one 
additional outcome of the more effective intervention under consideration. Like the average ratio, the method of computation for both the ICER and ICUR are identical, except the denominator for ICUR were based on the difference in QoL scores between two groups whereas the ICER was based on the difference in natural-unit outcome between two groups (e.g., difference in RTW rates between two groups). The incremental ratio is defined by the equation 5, based on the comparator group (A) and the group with the next highest outcome (B):

- Incremental Ratio $_{\mathrm{B}}=\frac{\left(\text { Average Cost }_{\mathrm{B}}-\text { Average Cost }_{\mathrm{A}}\right)}{\left(\text { Average Outcome }_{\mathrm{B}}-\text { Average Outcome }_{\mathrm{A}}\right)}$ (Eq. 5)

Parameter Uncertainty. The non-parametric bootstrap method was utilized to generate a joint distribution of cost and outcome variables, thus providing a probability distribution of the parameter estimate for the cost-effectiveness ratios (Chernick, 1999). This method involved 2,000 samples for each of the disability duration groups, with each sample consisting of 373 observations (the sample size of each group) sampled with replacement. Parameter uncertainty surrounding the average cost-effectiveness ratios were expressed using 95\% confidence intervals, constructed using the percentile method by taking the $2.5^{\text {th }}$ and the $97.5^{\text {th }}$ percentile values of the resulting bootstrapped distribution of ratios. For the incremental cost-effectiveness ratios, uncertainty was demonstrated using cost-effectiveness acceptability planes based on the distribution of bootstrapped estimates. Each plane described the incremental cost versus the incremental effectiveness of one group relative to another (e.g., ER group vs. DR group, ER group vs. ID group, ID group vs. DR group). The probabilities associated with cost- 
savings and greater effectiveness was indicated using the four quadrants of the plane, corresponding to being "more costly, more effective" (quadrant 1), "more costly, less effective" (quadrant 2), "less costly, less effective" (quadrant 3), and "less costly, more effective" (quadrant 4).

Total Cost of Illness. A basic Cost Analysis was provided to document the overall cost of illness associated with the disability duration groups, including a breakdown of costs associated with medical care, disability benefits, and lost productivity, in addition to the costs associated with the analytical timeframe (program costs and one-year post rehabilitation costs). This Cost Analysis was based on the conventional univariate data analysis techniques as discussed above.

Adjustment for Inflation. It should also be noted that all cost data described above were adjusted to the base year of 2005 U.S. Dollars. Cost adjustments were computed using both the general and medical care components of the Consumer Price Index (CPI), provided by the U.S. Bureau of Labor Statistics. The general CPI index was used to adjust all costs associated with disability benefits and lost productivity. The medical care CPI component index was used to adjust all costs associated with medical care and services. All adjustments, for each year (X), were computed using the following equation:

- $\operatorname{Cost}_{2005}=\operatorname{Cost}_{\mathrm{X}} *\left(\frac{\mathrm{CPI}_{2005}}{\mathrm{CPI}_{\mathrm{X}}}\right)$ (Eq. 6) 


\section{CHAPTER 3}

\section{RESULTS \\ Demographic and Pre-Rehabilitation Characteristics}

\section{Program Completion}

Prior to constructing the matched cohort, program completion rates were analyzed on the full cohort of eligible patients $(N=2,454)$. Tables A.5 and A.6 summarize the program completion rates as a function of disability duration. The results indicated that there was a significant difference in program completion rates across the three groups. The program completion rates were $79 \%(N=683), 77 \%(N=608)$, and $71 \%(N=574)$ for the ER, ID and DR groups, respectively, and were significantly different among groups. Specifically, post-hoc tests against the ER group revealed that the DR group was 1.6 times $(95 \%$ CI: $1.3,2.0)$ less likely to complete the program. However, no significant difference in completion rate was indicated for the ID group, relative to the ER group. The implications for non-completion and possible reasons of lower completion rate in the DR group are elaborated upon in the discussion. All the following analyses are based on the final matched cohort of program completers.

\section{Demographic Variables}

Table A.7 summarizes the demographic variables of the cohort. In addition to the four variables matched across the cohort, other demographic variables included marital status, level of education, and average length of disability in months. None of 
the variables significantly differed among the three disability duration groups, except for length of disability. Both the DR group (M: 37.5 months; $S D: 28.0$ months; $d: 5.2$ ) and the ID group ( $M: 13.0$ months; $S D: 2.8$ months; $d: 2.6)$ were significantly different from the ER group ( $M: 5.4$ months; $S D: 1.5$ months), as indicated by post-hoc tests.

\section{Pre-Rehabilitation Utilization Rate for Medical Services}

Table A.8 summarizes the utilization rates for medical care utilization in the PRIDE cohort. Table A.9 summarizes the effect sizes for significant differences between the groups observed in post-hoc tests. Significant differences among the groups were observed on the percentage of patients receiving any surgeries. Post-hoc analyses revealed that, compared to the ER group, the DR group was 9.9 times (95\% CI: 7.0 , 14.0) more likely to have any surgeries. Similarly, the ID group also significantly differed from the ER group, and was 4.4 times $(95 \% \mathrm{CI}: 3.2,6.2)$ more likely to have had any surgeries. Significant differences were also observed on the total number of surgeries undergone in each group. The DR group was significantly more likely to have two or more surgeries, at $4.4(95 \% \mathrm{CI}: 2.2,8.8)$ times, relative to the ER group. Although a significant linear trend was observed for increasing number of surgeries as disability duration increased, the ID group did not reach statistical significance for differences relative to the ER group. As noted in the Methods section, data on the utilization rates for other non-surgical medical services was unreliable. Therefore, only descriptive data are presented for these variables, since interpretation of any $p$-values on group differences are very likely misleading. 


\section{Occupational and Disability-related Characteristics}

In terms of the occupational characteristics of the cohort prior to injury, all three groups were not significantly different on type of occupation, physical demand levels of the occupation, and pre-injury average weekly wage levels. Additionally, no significant differences among the groups were observed on the percentage of patients who had returned to some level of light or modified duty during the duration between injury and admission to the functional restoration program. However, there was a significantly decreasing likelihood of having the original job available, as disability duration increased. Specifically, the DR group was 3.3 times $(95 \%$ CI: $2.5,5.0)$ less likely to have the original job available, relative to the ER group. Similarly, the ID group also significantly differed from the ER group, being 2.5 times (95\% CI: $1.7,3.3)$ less likely to have the original job available. Context for this finding is provided by significant differences in the duration of work missed due to disability, or temporary total disability (TTD), with both the DR group (M: 23.7 months; SD: 18.4 months; $d: 2.4$ ) and the ID group (M: 10.0 months; $S D: 4.7$ months; $d: 1.4$ ) significantly different from the ER group (M: 3.8 months; SD: 2.3 months).

In terms of the Workers' Compensation jurisdiction that patients were covered under, the majority of all three groups were from the Texas Workers' Compensation system, with no significant differences among the groups. However, an increasing trend towards case closure was observed as disability duration increased. Specifically, the DR groups was 14.0 times (95\% CI: 9.1, 21.5) more likely to have their Workers'

Compensation case closed, relative to the ER group. A similarly significant finding was 
observed for the ID group, which was 3.0 times $(95 \%$ CI: $1.9,4.7)$ more likely to have case closure relative to the ER group. A significant difference was observed on the proportion of patients being on SSDI at pre-rehabilitation, specifically only for the DR group which was 22.2 times (95\% CI: 3.0, 165.9) times more likely to be on SSDI, relative to the ER group. Finally, significant differences among the groups were indicated for the rate of attorney retention. Specifically, the DR group was 2.6 times (95\% CI: $1.7,3.8)$ more likely to have attorney representation, relative to the ER group. For the ID group, the patients were 2.4 times $(95 \%$ CI: $1.6,3.6)$ more likely to retain an attorney, relative to the ER group. Table A.10 summarizes the overall results for these occupational characteristics of the cohort, while Table A.11 summarizes the effect size magnitudes of post-hoc tests conducted on statistically significant occupational variables.

Pre-Rehabilitation Psychiatric Diagnoses

Significant differences among the groups were indicated for several of the PostInjury DSM-IV psychiatric diagnoses. Overall, a significant, and linearly increasing trend was observed for the prevalence of Any Axis I diagnoses (excluding Pain Disorder) as disability duration increased. Specifically, the DR group was 2.8 times (95\% CI: 2.0, 3.9) more likely to have an Axis I disorder, relative to the ER group. Additionally, the ID group also significantly differed in terms of Axis I prevalence rates, being 2.2 times $(95 \%$ CI: 1.6, 3.0) more likely to have an Axis I disorder, relative to the ER group. No significant differences were observed on prevalence of Axis II disorders among the groups. However, both the ID and DR groups were significantly 
more likely to have combined Axis I and II disorders, being 1.6 times (95\% CI: 1.2, 2.2) and 1.8 times $(95 \% \mathrm{CI}: 1.3,2.5)$ respectively, relative to the ER group.

Significant differences among the groups were also observed on the individual diagnostic categories. Both the ID and DR groups evidenced significantly higher rates of Major Depressive Disorder (MDD), relative to the ER group. The DR group was 2.2 times (95\% CI: 1.6, 3.0) more likely to have MDD, relative to the ER group. A similar odds of suffering from MDD was observed for the ID group, being 2.1 times $(95 \% \mathrm{CI}$ : $1.5,2.8)$ more likely to reach threshold for clinical depression, relative to the ER group. Furthermore, significantly higher rates of post-injury alcohol abuse and opioid dependence were observed for the DR group, relative to the ER group. Patients in the DR group were 5.6 times (95\% CI: 1.2, 25.5) more likely to have abused alcohol, and 2.6 times $(95 \%$ CI: $1.8,3.9)$ more likely to be dependent on opioids, relative to the ER group. In terms of non-opioid drug dependence disorder, the significant overall effect was not qualified by post-hoc pairwise comparisons against the ER group, with comparisons failing to yield a significant $p$-value. Finally, all three groups were not significantly different in the prevalence rates on any of the Axis II Personality Disorders. Table A.12 summarizes the findings on post-injury DSM-IV psychiatric diagnoses among the three groups, while Table A.13 summarizes the effect sizes from post-hoc analyses of significant findings on post-injury DSM-IV psychiatric diagnoses. Pre-Rehabilitation Psychosocial Measures

Table A.14 summarizes the results of the analyses on the pre-rehabilitation psychosocial measures, and Table A.15 summarizes the effect sizes for significant 
differences between groups after post-hoc analyses. Among the psychosocial measures administered to the patients, only two measures were significantly different among the three groups. The HAM-D revealed significant differences in clinician ratings of depression among the groups, with a significant linear trend for increasing disability duration. Post-hoc tests indicated both the DR group (M: 18.0; SD: 5.4; $d: 0.4)$ and the ID group ( $M: 17.5 ; S D: 5.7 ; d: 0.3)$ significantly differed from the ER group (M: 15.6; $S D: 5.8)$. When analyzed within established clinical norms for having moderate-tosevere depressive symptoms (HAM-D $\geq 14$ ), both he ID and DR groups had a significantly greater percentage of reaching this clinical threshold. Specifically, the DR group was 2.1 times $(95 \%$ CI: $1.5,3.0)$ more likely to have moderate-to-severe depressive symptoms relative to the ER group. The ID group had a lower effect size magnitude on this measure, being 1.6 times $(95 \% \mathrm{CI}: 1.1,2.1)$ more likely to demonstrate moderate-to-severe depressive symptoms, relative to the ER group.

The MVAS indicated overall significant differences among groups as well as a significant linear trend of poorer MVAS scores with increasing disability duration. However, post-hoc tests indicated that only the DR group (M:96.8; SD:23.8; $d: 0.2)$ differed significantly from the ER group ( $M$ : 90.7; $S D$ : 26.9). In terms of a clinical cutoff score for severe disability (MVAS $\geq 101$ ), the DR group significantly differed from the ER group and was 1.5 times $(95 \%$ CI: $1.1,2.0)$ more likely to have severe disability scores. Finally, no significant differences among groups were observed on pain intensity, any of the SF-36 composite scores and subscales, as well as on the utilitybased quality of life measure. 
Treatment Efficacy of the Functional Restoration Program

\section{Post-Rehabilitation Psychosocial Measures}

At post-rehabilitation, none of the psychosocial measures evidenced a significant effect among the groups. It should be noted that all post measures were analyzed using the ANCOVA, with the corresponding pre-rehabilitation measure included as a covariate. Thus, these findings are null, even after using a relatively more powerful analyses. However, a significant linear trend was still observed for increasing depressive symptoms on the HAM-D, that when analyzed at the clinical threshold (HAM-D $\geq 14)$, indicated that the DR group was 1.8 times $(95 \%$ CI: $1.2,2.9)$ more likely to have moderate-to-severe depressive symptoms at post-rehabilitation, relative to the ER group. Finally, a significant linear trend was also observed for a slightly increasing percentage of patients across the disability groups reporting severe functional limitations on the MVAS at post-rehabilitation, but with no overall significant differences among the groups. Table A.16 summarizes the post-rehabilitation measures for the three groups.

\section{One-Year Socioeconomic Outcomes}

One-year socioeconomic outcomes are summarized in Table A.17, while the effect size measures for significant differences in disability compensation are summarized in Table A.18. At one-year post-rehabilitation, all groups fared extremely well on the objective socioeconomic outcomes. Other than a significantly decreasing linear trend for work retention at one-year, no significant differences were observed among the groups on occupational status and amount of healthcare utilization. 
Additionally, re-injury and case closure rates across the three groups were similar. However, patients in the late rehabilitation groups were more likely to be still receiving disability benefits at one-year post-rehabilitation. Specifically, both the ID and DR groups were significantly more likely to be receiving Impairment Income Benefits, at 2.9 times $(95 \%$ CI: $1.4,6.0)$ and 3.3 times $(95 \%$ CI: $1.6,6.9)$ respectively, relative to the ER group. Additionally, both groups were also more likely to be on SSDI at oneyear post-rehabilitation. The ID group was 14 times (95\% CI: 1.8, 107.5) more likely to be on SSDI, than the ER group. For the DR group, patients were 30.9 times $(95 \% \mathrm{CI}$ : 4.2, 228.6) more likely to receive SSDI, relative to those patients in the ER group. Finally, significant differences were also observed on the receipt of Supplemental Income Benefits at one-year. Specifically, the DR group was 21.8 times (95\% CI: 2.9 , 163.0) more likely to be on SIBS at one-year post-rehabilitation, relative to the ER group.

\section{Cost-Effectiveness Analysis of the Functional Restoration Program}

\section{Costs of the PRIDE Functional Restoration Program}

Table A.19 summarizes the total costs as well costs of the various components of the PRIDE Functional Restoration Program, while Table A.20 summarizes the effect sizes for significant differences indicated in post-hoc tests. An overall significant, but small, effect was observed for total costs associated with the PRIDE program, among the three disability duration groups. Specifically, the ID group (M: $\$ 30,342 ; S D: \$ 4,608$; $d: 0.3$, with the highest cost among the three groups, was found to significantly differ from the ER group ( $M: \$ 28,994 ; S D: \$ 5,072)$, as indicated by the post-hoc test. 
Similarly, the total PRIDE program costs for DR group (M: $\$ 30,195 ; S D$ : $\$ 6,294 ; d$ :

0.2 ) was also found to be significantly higher than the ER group.

The total costs of the PRIDE functional restoration program was further broken down into several components associated with the costs of treatment hours of the program, costs of consultation and doctors' office visits at the PRIDE facility, costs of any therapeutic injections received, and the costs of medications prescribed during admission to, and discharge from, the program. Total treatment hours for the functional restoration program significantly differed among the three groups, accompanied by a significant linear trend of increasing treatment hours associated with increased disability duration. Post-hoc tests indicated that both the ID group (M: 176 hours; SD: 26.8 hours; $d: 0.5$ ) and the DR group ( $M: 177$ hours; $S D: 34.6$ hours; $d: 0.6$ ) were associated with relatively longer treatment durations compared to the ER group ( $M$ : 160 hours; $S D: 28.4$ hours). In terms of the costs associated with these treatment hours, similarly significant results were observed for overall group differences accompanied by a significant linear trend for increasing costs associated with increased disability duration. Both the ID group (M: $\$ 25,997 ; S D$ : $\$ 3,916 ; d: 0.3)$ and the DR group (M: $\$ 26,188 ; S D: \$ 5,683 ; d$ : 0.3 ) had significantly higher costs relative to the ER group ( $M$ : $\$ 24,499 ; S D$ : $\$ 4,330)$, as indicated by post-hoc tests.

For the costs of consultation and doctors' office visits, overall significant differences were indicated among the three groups, with a linear trend of decreasing costs associated with increasing disability duration. Post-hoc tests indicated that only the DR group ( $M: \$ 3,557 ; S D: \$ 1,507 ; d:-0.4)$ significantly differed from the ER group 
( $M: \$ 4,145 ; S D: \$ 2.230)$ for this component of the total costs associated with the PRIDE program.

In terms of the costs of prescribed medications at admission and discharge, both sets of analyses revealed a significant overall difference among groups, accompanied by significant linear trends for increasing costs of prescription medications for longer durations of disability. Post- hoc tests on the cost of admission medications indicated that both the ID group (M: \$257; SD: \$160; $d: 0.3)$ and DR group (M: $\$ 267 ; S D: \$ 162$; $d: 0.3$ ) had significantly higher costs, relative to the ER group ( $M: \$ 215 ; S D: \$ 160)$. For costs associated with discharge medication, post-hoc tests revealed that only the DR group (M: \$272; SD: \$176; d: 0.3) significantly differed from the ER group (M: \$226; $S D: \$ 198)$.

Finally, no significant differences were observed among the three disability duration groups for the small number of patients who received injection therapeutics as part of the PRIDE rehabilitation program.

\section{One-Year Post-Rehabilitation Costs}

Table A.21 summarizes the costs incurred during the one-year postrehabilitation period. Costs associated healthcare utilization at during this period consisted of estimated costs of visits to new healthcare providers for the original injury, as well as estimated costs for any new surgeries to the injured regions of the body associated with the original injury claim. Consistent with the non-significant findings among the groups as described in the analyses of one-year outcomes, no significant 
differences among the groups were observed for the costs associated with visits to new healthcare providers and costs for new surgeries for the original injury.

\section{Total Costs within the Analytical Timeframe}

The total costs within the analytical timeframe for the full economic evaluation consisted of the program costs and the one-year post-rehabilitation costs. Tables A.22 and A.23 summarize the costs within the analytical timeframe for each of the three disability duration groups. Significant differences were observed among the groups. Specifically, post-hoc tests indicated that both the DR group ( $M$ : $\$ 30,553 ; S D$ : $\$ 6,818$; $d: 0.3)$ and the ID group ( $M: \$ 30,700 ; S D: \$ 5,143 ; d: 0.2)$ significantly differed from the ER group (M: $\$ 29,255 ; S D: \$ 5,301)$.

\section{CEA Based on Return-to-Work}

Table A.24 presents the Average Cost Effectiveness Ratios (ACER) for the outcome of RTW. The average cost per unit outcome of RTW for the ER group was $\$ 32,711$ (95\% CI: $\$ 31,390$ - $\$ 34,206)$. The average cost per unit outcome of RTW for the ID group was $\$ 34,765$ (95\% CI: $\$ 33,255$ - $\$ 36,345)$. Finally, for the DR group, the average cost per unit outcome of RTW was $\$ 36,051$ (95\% CI: $\$ 34,264$ - \$38,080). As indicated by the overlapping confidence intervals, the average cost per unit outcome of RTW does not significantly differ between the ER and ID groups, as well as between the ID and DR groups. However, it should be noted that a small portion of non-overlap exists between the $95 \%$ confidence interval of the DR group, relative to the ER group.

Table A.25 summarizes the cost-effectiveness analysis for RTW based on the Incremental Cost-Effectiveness Ratio (ICER). The first step of the analysis yielded an 
ICER indicating an incremental cost of $\$ 4,130$ per additional injured worker returned to work for the ID group, relative to the DR group. The incremental cost-effectiveness plane of the ID group relative to the DR group is illustrated in Figure B.1. Within the same level of analysis, the ER group dominated the ID group (i.e., a negative ICER value of $-\$ 128,353)$, demonstrating unquestionable cost-effectiveness compared to the ID group. Figure B.2 illustrates the incremental cost-effectiveness plane of the ER group relative to the DR group. The next level of analysis, with the dominated ID group removed, compares the ER group to the DR group. The negative ICER of $-\$ 27,703$ indicates a strong domination of the ER group over the DR group, with the corresponding incremental cost-effectiveness plane illustrated in Figure B.3.

Given the strong domination of the ER group over the ID and DR groups, as well as the narrow range of incremental effectiveness that results in division by zero in some of the individual bootstrap estimates, confidence intervals cannot be reliably constructed for the incremental ratios of each comparison. Therefore, Table A.26 summarizes the probability of the bootstrap estimates within each quadrant of the incremental cost-effectiveness planes accompanying each comparison. These values represent the probability of a given strategy (i.e., early versus delayed rehabilitation) being cost-effective, based on the distribution of the bootstrapped estimates along the four quadrants of the plane.

Overall, the probability distribution indicated with a certainty that early rehabilitation is cost-saving. Compared to the DR group, the ER group was almost certainly likely (96\%) to be less costly and more effective. For approximately $32 \%$ of 
the estimates, the ER group was identified as being less costly than the ID group, but also less effective at the same time.

For the comparison between the ID and DR groups, the majority of estimates $(92 \%)$ indicated that rehabilitation during the intermediate duration of $9-18$ months was more effective than delayed rehabilitation. However, $58 \%$ of these estimates were also identified as being more costly, thus accounting for the positive ICER value reported in Table A.25.

\section{CEA Based On Work Retention}

Table A.27 presents the Average Cost Effectiveness Ratios (ACER) for the outcome of work retention at one-year. The average cost per unit outcome of work retention for the ER group was $\$ 35,442$ (95\% CI: $\$ 33,563$ - \$37,599). The average cost per unit outcome of work retention for the ID group was $\$ 37,300$ (95\% CI: $\$ 35,311$ $\$ 39,392)$. Finally, for the DR group, the cost per unit outcome of work retention was \$40,201 (95\% CI: \$37,503 - \$43,131). The overlapping confidence intervals indicate that the average cost per unit outcome of work retention does not significantly differ among all three groups.

Table A.28 summarizes the cost-effectiveness analysis for work retention based on the Incremental Cost-Effectiveness Ratio (ICER). The first step of the analysis yielded an ICER for the ID group that indicated an incremental cost of $\$ 2,480$ per additional injured worker retaining work at one-year, relative to the DR group. Figure B.4 illustrates the incremental cost-effectiveness plane for the comparison between the ID and DR groups. Within the same level of analysis, the ER group dominated the ID 
group (i.e., a negative ICER value of $-\$ 606,379)$, therefore demonstrating unquestionable cost-effectiveness compared to the ID group, for the outcome of work retention. Figure B.5 illustrates the incremental cost-effectiveness plane for the ER group relative to the ID group. The next level of analysis, with the dominated ID group removed, compares the ER group to the DR group. Once again, the negative ICER of $\$ 18,543$ indicates a strong domination of the ER group over the DR group. The accompanying incremental cost-effectiveness plane for this comparison is illustrated in Figure B.6.

Due to similar circumstances with the analysis using RTW as the measure of effectiveness, confidence intervals cannot be reliably constructed for the incremental ratios of each comparison for work retention. Therefore, Table A.29 summarizes the probability distribution of the bootstrap estimates observed within each quadrant of the incremental cost-effectiveness plane. Compared to the DR group, the ER group was, once again, almost certainly likely to be less costly and more effective with $98 \%$ of the estimates indicating early rehabilitation as a less costly, and more effective strategy for rehabilitation. When compared to the ID group, effectiveness for early rehabilitation was an almost even split (46\% less effective, 54\% more effective). However, both cases demonstrated with a certainty that early rehabilitation is cost-saving.

Comparable to the results obtained for RTW, the comparison between the ID and DR groups on work retention indicated that the vast majority of estimates (98\%) associated with the intermediate duration of $9-18$ months were more effective than 
delayed rehabilitation. However, as indicated by the positive ICER value previously, $62 \%$ of these estimates were also identified as being more costly.

\section{CUA Based On Post-Treatment Quality of Life}

Table A.30 presents the Average Cost Utility Ratios (ACUR) for the outcome of post-treatment changes in quality of life (QoL), accompanied by the average of the bootstrap estimates for the joint distribution of costs and change in QoL for each of the three disability duration groups. The average cost per unit QoL score gained for the ER group was $\$ 585,100$ (95\% CI: $\$ 546,752$ - $\$ 764,108)$. The average cost per unit QoL score gained for the ID group was $\$ 614,000$ (95\% CI: $\$ 532,920$ - \$706,145). Finally, for the DR group, the cost per unit outcome of work retention was $\$ 611,060$ (95\% CI: $\$ 574,519$ - \$775,667). The overlapping confidence intervals indicate that the average cost per unit QoL score gained does not significantly differ among all three groups. Due to similarities in the QoL change scores for each group (Table A.31), Incremental Cost Utility Ratios (ICURs) could not be computed due to the problem of division by zero. Predictors of Costs in the Analytic Timeframe

Pre-rehabilitation variables significantly differing among the disability duration groups were utilized as predictors in a sequential regression analysis on the total cost associated with the analytical timeframe of the study. These variables included depressive symptoms measured by the HAM-D, perceived disability measured by the MVAS, Major Depressive Disorder, Alcohol Abuse Disorder, Opioid Dependence Disorder, Non-opioid Drug Dependence Disorder, job availability, attorney retention, Workers' Compensation case closure, and prior surgical procedures. Prior to running 
the regression analysis, all predictor variables were evaluated in a correlation matrix to ensure no problems with multicollinearity were present. Table A.32 summarizes the correlations among the predictor variables, and indicates no redundancy among the variables (all $r$ 's $<.80)$.

Table A.33 summarizes the results of the sequential regression analysis. After adjusting for the three disability duration groups (with ER as the reference group), the variables identified as significant predictors of costs in the analytical timeframe included scores on the MVAS, the diagnosis of Major Depressive Disorder, Workers' Compensation case closure, and the presence of surgery. The final model of the regression analysis, including only the significant variables and after adjusting for disability duration, are presented in order of magnitude for their unique effect on costs. Both case closure $(\beta=-0.13)$ and the diagnosis of Major Depressive Disorder $(\beta=0.13)$ had the largest effect on costs. Case closure was associated with lower costs during the analytical timeframe, while depression was positively associated with costs. The next strongest predictor of cost was the presence of any surgeries received prior to rehabilitation $(\beta=0.10)$. Being disabled more than 18 months was also positively associated with costs $(\beta=0.09)$, and this was followed in magnitude of importance by perceived disability as measured by the MVAS $(\beta=.08)$ and being disabled between 9 -18 months $(\beta=.07)$, both of which were also positively associated with costs during the analytical timeframe. 


\section{Cost Analysis on the Total Cost of Illness}

\section{Cohort from Ohio Bureau of Workers' Compensation}

The second column in Table A.34 describes the characteristics of the initial cohort from OBWC in terms of age, gender, injured musculoskeletal regions of the body, the prevalence of spinal and non-spinal surgeries associated with the injury claim, and length of disability. In order to obtain reliable estimates on medical costs, a further refining of this cohort was undertaken in order to match the PRIDE cohort on relevant variables associated with costs. Variables selected to be category matched on the PRIDE cohort were injured musculoskeletal regions and type of surgery (spinal and non-spinal musculoskeletal surgeries). The initial matched cohort from OBWC is displayed in the third column of Table A.34.

Prior to finalizing the estimates, the data were screened for any abnormalities. Figures B.7, B.8, and B.9 display histograms of total medical costs, rescaled using the logarithmic transformation, for each of three disability duration groups from the OBWC. It should be noted that examining the rescaled data on costs allow for more accurate detection of abnormalities in the pattern of data. While the first two disability duration groups (Figures B.7 and B.8) have an approximately normal distribution, the extreme disability group (Figure B.9) was relatively bimodal in shape, suggesting two different populations of patients in this group. By observing the values of the log scale on the $\mathrm{x}$-axis of the figures, it is obvious that this sub-group of patients in the extreme disability group had substantial amounts of lower costs that were not representative of the distributions of all three disability duration groups. This is further illustrated in 
Figure B.10, using box plots of the rescaled total costs for the three groups, and by examining the percentile values for total costs (in dollars) for the three groups as displayed in Table A.35. On further examination, this was a small group of $N=194$ patients, whose costs were almost exclusively from physician consults. The decision was made to exclude these patients from the OBWC cohort, given that the costs associated with these patients were unrepresentative and untypical of not only the extended disability group from OBWC, but also when compared to the other two disability duration groups. After removing the outliers, the remaining $N=925$ patients from the OBWC cohort were used to estimate medical costs based on duration of disability. The fourth and fifth columns from Table A.34, presented previously, summarize the final selected cohort from OBWC and the cohort of PRIDE patients, respectively. It should be noted that only a small number of PRIDE patients fall outside the range of disability duration present in the OBWC cohort $(N=6$; approximately $1 \%$ within the DR group; $<0.1 \%$ overall).

To ensure that final medical cost estimates were not confounded with differences in the distribution of gender and age between the PRIDE and OBWC cohorts, Table A.36 presents the correlations among rescaled total costs, age and gender within the OBWC cohort. Neither age nor gender had a significant correlation with total costs.

Medical Cost Estimates - by Service Providers

Table A.37 summarizes estimated medical costs associated with disability duration groups of $4-8$ months, $9-18$ months, and greater than 18 months. Table 
A.38 presents the effect size magnitudes for significantly different findings observed in post-hoc analyses. For ease of presentation, these groups will simply be referred to as the ER, ID, and DR group, respectively, given that the costs presented here constitute estimates for the disability duration groups in the PRIDE cohort. Overall, significant differences in total medical costs were observed among the groups. Specifically, in the post-hoc tests, the DR group ( $M$ : $\$ 67,612, S D$ : $\$ 75,793 ; d: 1.1)$ differed significantly with a large effect size, relative to the ER group ( $M$ : \$21,356; SD: $\$ 21,204)$. The ID group ( $M: \$ 33,750 ; S D: \$ 25,725 ; d: 0.6$ ) also significantly differed from the ER group, with a medium-to-large effect size magnitude.

The costs accrued for almost all professional services were also significantly different among the groups. The biggest differences in costs, with significant findings between both extended disability groups and the shorter-term disability group, were observed in physician, pharmacy and case management costs. In terms of costs associated with physician services, post-hoc tests revealed that both the ID group ( $M$ : $\$ 8,071 ; S D: \$ 7,940 ; d: 0.4)$ and the DR group (M: $\$ 16,057 ; S D: \$ 14,277 ; d: 1.0)$ differed significantly from the ER group ( $M$ : $\$ 5,457 ; S D$ : $\$ 5,432)$. Similarly significant findings were observed for pharmacy costs and case management services. In terms of pharmacy costs, post-hoc tests indicated that the both the ID group ( $M: \$ 4,284 ; S D$ : $\$ 8,491 ; d: 0.3)$ and the DR group ( $M: \$ 11,818 ; S D: \$ 17,640 ; d: 1.1)$ differed significantly from the ER group ( $M$ : $\$ 2,270 ; S D$ : $\$ 4,392$ ). Relative to the ER group ( $M$ : $\$ 1,924 ; S D$ : $\$ 1,605)$, case management costs were also significantly higher in the ID group (M: $\$ 3,761 ; S D: \$ 3,360 ; d=0.5)$, as well as in the DR group ( $M: \$ 4,767 ; S D$ : 
$\$ 4,671 ; d=0.7)$. It should be noted that all three categories of costs were also associated with a significant linear trend of increasing costs with disability duration.

Several categories of costs with significant, but less substantial, differences among the groups were observed in the categories of costs associated with hospital services, psychological services, and physical and occupational therapists. Although an overall significant effect and a linear trend of increasing hospital-associated costs were observed as disability duration increased, post-hoc tests revealed that only the DR group ( $M: \$ 26,526 ; S D: \$ 48,396 ; d: 0.5)$ significantly differed from the ER group ( $M$ : $\$ 10,203 ; S D$ : $\$ 14,189)$. Similar findings were also observed for the cost of services provided by psychologists and licensed professional counselors. Specifically, only the DR group ( $M: \$ 3,414 ; S D: \$ 3,922 ; d: 0.8)$ significantly differed from the ER group ( $M$ : $\$ 1,658 ; S D$ : $\$ 3,581)$, despite a significant linear trend of increasing costs of psychological services across the disability duration groups. For the category of costs associated with physical and/or occupational therapists, a contrast with previous cost categories were observed when post-hoc tests revealed that only the ID group ( $M$ : $\$ 5,808 ; S D: 5,859 ; d: 0.5)$ significantly differed from the ER group ( $M: \$ 3,231 ; S D$ : $\$ 3,405)$. The single remaining significant finding, in terms of costs associated with other types of service providers, also yielded a significant difference during post-hoc tests with the DR group $(M: \$ 5,170 ; S D$ : $\$ 19,059 ; d$ : 0.7$)$ indicating significantly higher costs compared to the ER group ( $M$ : $\$ 1,561 ; S D$ : $\$ 2,126)$.

Finally, no significant differences among the groups were observed on costs associated with chiropractors, pain management centers, and job retraining specialists. 
However, a significant linear trend of increasing costs associated with job retraining was observed as disability duration increased. It should also be noted, that, all costs described and analyzed above sum to the total medical costs associated with the disability claim presented in the first paragraph of this section.

Medical Cost Estimates - by Specific Medical Interventions and Procedures

Cost associated with several major categories of medical interventions and procedures were extracted from the total costs presented in the previous section. Table A.39 summarizes these specific costs and the results of the analyses for each disability group, while Table A.40 summarizes the effect sizes for significant differences indicated by post-hoc tests following the main analyses.

Cost categories associated with consultation, medication, and diagnostic procedures were significantly different among all three groups, accompanied by a significant linear trend of increasing costs with disability duration. Both the ID group (M: \$1,954; SD: \$1,865; $d: 0.6)$ and the DR group (M: \$3,728; SD: 2,832; d: 1.3) significantly differed from the ER group $(M: \$ 1,196 ; S D$ : $\$ 1,251)$ in post-hoc tests on costs associated with consultation. Post-hoc tests on medication costs for the ID group (M: \$4,281; SD: \$8,492; $d: 0.3)$ and the DR group (M: $\$ 11,802 ; S D: \$ 17,651 ; d: 1.1)$ were both significantly different from the ER group ( $M: \$ 2,275 ; S D: 4,403)$. For costs associated with diagnostic procedures, post-hoc tests similarly indicated significant differences for the ID group (M: $\$ 1,214 ; S D: 1,176 ; d: 0.3)$ and DR group (M: $\$ 2,482$; $S D: \$ 2,618 ; d: 0.8)$, relative to the ER group (M: $\$ 859 ; S D: \$ 875)$. 
Costs associated with injection therapeutics and anesthesiology services indicated an overall significant effect, accompanied by a linear trend of increasing costs associated with length of disability. However, post-hoc tests on costs associated with injection therapeutics revealed that only the DR group $(M: \$ 2,278 ; S D: \$ 6,160 ; d: 0.7)$ significantly differed from the ER group ( $M$ : \$614; $S D$ : \$1,109). Similarly, for costs associated with anesthesiology services, only the DR group (M: $\$ 1,298 ; S D$ : $\$ 1,196 ; d$ : 0.5 ) differed significantly from the ER group ( $M: \$ 774 ; S D$ : $\$ 558)$, as indicated by posthoc tests.

Costs associated with the surgeon's cost for both spinal and non-spinal surgeries, primary and secondary care modalities (PM\&R modalities), as well the costs of work hardening and work conditioning programs were all not significantly different among groups. Furthermore, there was no significant linear trend associated with these costs across the disability duration groups.

Estimated Productivity Losses and Disability Benefits

Table A.41 summarizes the costs associated with productivity losses and accrued disability benefits for each of the three disability duration group, while the effect sizes for significant differences indicated in post-hoc tests are summarized in Table A.42. Large and significant differences were observed among all three groups for the cost of lost productivity as well as total accrued cost of disability benefits. Both the ID group (M: $\$ 32,510 ; S D: \$ 33,465 ; d: 1.0)$ and the DR group (M: $\$ 73,075 ; S D$ :

$\$ 66,289 ; d: 1.9)$ differed significantly from the ER group ( $M: \$ 12,547 ; S D: \$ 13,604)$, as indicated by post-hoc tests on the cost of lost productivity. Similarly, post-hoc tests on 
the total cost of disability benefits indicated significant differences for the ID group ( $M$ : $\$ 18,411 ; S D: \$ 10,270 ; d: 1.3)$ and the DR group (M: $\$ 36,790 ; S D: \$ 25,028 ; d: 2.2)$, relative to the ER group ( $M: \$ 7,328 ; S D: \$ 4,776)$.

Total Disability Benefits were further broken down into benefits for Temporary Total Disability (TTD) that compensated for wage loss, as well as Impairment Income Benefits made payable by impairment ratings documenting reduced functional capacity associated with the injury. As described in the Methods section, TTD benefits were computed based on rules specific to the different types of Workers' Compensation jurisdictions under which the individual patients were associated with. In terms of the Texas Workers' Compensation system, Temporary Income Benefits (TIBS) payments had an overall significant effect across the disability duration groups, accompanied by a significant linear trend of increasing costs as disability duration increased. Specifically, the ID group (M: $\$ 16,810 ; S D: \$ 9,761 ; d: 1.2)$ and the DR group $(M: \$ 29,103 ; S D$ : $\$ 16,275 ; d: 1.9)$ both significantly differed from the ER group ( $M: \$ 6,916 ; S D: \$ 4,515)$, as indicated by post-hoc tests.

For injured workers under the Federal Workers' Compensation jurisdiction of the Federal Employees Compensation Act (FECA), the significant overall difference and linear trend associated with disability duration was accounted for in post-hoc tests, indicating both the ID group $(M: \$ 22,051 ; S D: \$ 10,646 ; d: 1.3)$ and the DR group (M: $\$ 45,897 ; S D$ : $\$ 46,106 ; d: 1.8)$ being significantly different from the ER group (M: $\$ 6,892 ; S D: \$ 3,170)$. 
Similar findings were observed for the small number of patients who were Texas Non-Subscribers. Specifically, post-hoc tests revealed that both the ID group (M: $\$ 14,281 ; S D: \$ 8,154 ; d: 1.4)$ and the DR group (M: $\$ 31,250 ; S D: \$ 3,972 ; d: 2.8)$ differed significantly from the ER group ( $M$ : $\$ 6,263 ; S D$ : $\$ 3,475)$, in terms of NonSubscriber TTD benefits.

For the small number of injured workers under the jurisdiction of out-of-state Workers' Compensation systems, an overall significant difference among the groups was also accompanied by a linear trend of increasing costs of disability benefits across disability duration groups. However, post-hoc tests revealed that only the DR group ( $M$ : $\$ 64,751 ; S D: \$ 55,683 ; d: 2.3)$ significantly differed from the ER group (M: $\$ 7,575 ; S D$ : $\$ 3,583)$.

Finally, a significant overall effect for differences in costs associated with Impairment Income Benefits was also accompanied by a significant linear trend for increasing costs as disability duration increased. However, post-hoc tests revealed that only the DR group (M: $\$ 14,839 ; S D: \$ 8,820 ; d: 0.9)$ was significantly different from the ER group (M: $\$ 8,290 ; S D: \$ 3,324)$.

\section{Total Cost of Illness}

The estimated cost of illness over the entire duration of disability includes the sum of the estimated medical costs, estimated disability benefits, estimated productivity losses, and the costs associated with the analytical timeframe of the functional restoration program (program costs + one-year post-rehabilitation costs). Table A.43 summarizes the estimated total cost of illness associated with this cohort. 
Given the disparity in the total costs incurred over the duration of disability for each of the groups, substantial cost savings are indicated for early intensive, interdisciplinary rehabilitation based on the functional restoration program. Relative to the DR group, the average savings in medical costs over the duration of disability is $\$ 46,256$ or approximately $68 \%$ in costs saved when rehabilitation is administered within $4-8$ months post-injury. In a comparison of the ID group to the ER group, the average savings over the duration of disability amount to $\$ 12,394$ or approximately $37 \%$ in costs saved.

The cost of accrued disability benefits can be potentially reduced by $80 \%$ $(\$ 29,462)$, when early rehabilitation is administered, relative to allowing the duration of disability to be prolonged beyond 18 months. With respect to the ID group, potential savings in disability benefits are 60\% $(\$ 11,083)$, if rehabilitation is administered early within $4-8$ months post-injury. Similarly, the costs of lost productivity can be reduced by $83 \%$ over the duration of disability when comparing the DR group to the alternative of early rehabilitation as demonstrated by the ER group. For the ID group, savings in productivity losses correspond to $61 \%$, relative to the ER group. In terms of the costs over the duration of the functional restoration program, savings of $4 \%-5 \%(\$ 1,298-$ $\$ 1,445)$ are indicated for early rehabilitation, relative to late rehabilitation. Finally, in terms of the total cost of illness across the disability duration groups, a savings of $66 \%$ $(\$ 137,544)$ was indicated for early rehabilitation, relative to the DR group. For the ID group, potential savings in total costs over the duration of disability correspond to $39 \%$ $(\$ 44,885)$ if rehabilitation is administered early within $4-8$ months post-injury. 


\section{CHAPTER 4}

\section{DISCUSSION}

The present study was designed to address the issue of treatment efficacy as well as cost-effectiveness for the effect of disability duration on long-term outcomes among patients with Chronic Disabling Occupational Musculoskeletal Disorders (CDOMD), based on the Phase Model of Disability proposed by Krause and Ragland (1994). Specifically, the Phase Model of Disability proposed that patients disabled for greater than 18 months had little recourse for adequate recovery from disability. While an earlier study on a CDOMD cohort investigated the one-year outcomes following a course of functional restoration (Jordan, Mayer, \& Gatchel, 1998), the present study is the first, to date, incorporating a cost perspective in the design of the study. In addition, the present study differs from the previous one in using a matched cohort design, thus controlling for any confounding effects of demographic variables, such as age, gender, and ethnicity, as well as types of musculoskeletal injuries.

Given the major objective based on the Phase Model of Disability, the present study established four major goals in evaluating the effect of extended periods of disability due to CDOMD. Firstly, it was designed to evaluate the baseline characteristics as a function of disability duration, for patients who completed a functional restoration program for CDOMDs. Specific to this goal was the hypothesis that patients in the Early Rehabilitation (ER) group would demonstrate significantly less 
psychopathology and significantly more positive responses on functional and psychosocial measures at pre-rehabilitation, relative to patients in the Intermediate Duration (ID) and Delayed Rehabilitation (DR) groups. Secondly, it was designed to evaluate treatment efficacy of a functional restoration program for the three groups that differed in duration of disability. Specific to this goal were two hypotheses that predicted: (1) patients in the ER group would demonstrate significantly more positive responses on functional and psychosocial measures at post-rehabilitation, relative to patients in the ID and DR groups; and (2) that patients in the ER group would demonstrate significantly better responses on objective socioeconomic outcomes at oneyear post-rehabilitation. Thirdly, this study was also designed to establish the costeffectiveness of early rehabilitation, relative to conventional practices of providing intensive, interdisciplinary tertiary care only after prolonged durations of disability. Specific to this goal was the hypothesis that the ER group would demonstrate superior cost-effectiveness, relative to the ID and DR groups, both in terms of objective outcomes as well as in improvements in health-related quality of life. And finally, this study was also designed to document the accrued costs associated with duration of disability, and to provide an estimate of the total cost of illness incorporating costs associated with medical care, disability benefits, and lost productivity.

\section{Pre-Rehabilitation Characteristics}

The hypothesis on the baseline psychosocial characteristics was partially supported by the data. As expected, patients who presented for early rehabilitation in a functional restoration program demonstrated significantly less psychopathology. Of 
specific note is the prevalence of post-injury Axis I disorders. While the overall prevalence rate for post-injury Axis I disorders in a CDOMD cohort was previously established at 57\% (Dersh, Mayer, Theodore, Polatin, \& Gatchel, 2007), approximately three-fourths of the patients in the DR group were afflicted with Axis I disorders. With regard to the specific Axis I diagnoses, both the ID group and DR group demonstrated a doubling in the odds of reaching significant thresholds for being clinically depressed, relative to the ER group. Furthermore, patients with extended disability of greater than 18 months were also more likely to engage in alcohol abuse and develop dependence on narcotic analgesics. While opioid dependence is generally associated with longer durations of pain and disability (Dersh et al., 2008), the rate of alcohol abuse observed in the DR group was substantially higher than the prevalence rate of $<1 \%$ for a general CDOMD cohort (Dersh, Mayer, Theodore, Polatin, \& Gatchel, 2007). However, no clear causal evidence can be drawn between disability duration and alcohol abuse, given the non-randomized design of the study. Within this context, however, it should also be noted that the literature documents alcohol abuse being correlated with, and sometimes as a precursor to, opioid dependence disorder (Ives et al., 2006; Turk, Swanson, \& Gatchel, 2008). A similar confounding relationship for alcohol abuse has also been noted among patients with Major Depressive Disorder (Arnow et al., 2006).

Consistent with the findings on Major Depressive Disorder, a substantially greater amount of patients in the late rehabilitation groups were observed with moderate-severe levels depressive symptoms as measures by the Hamilton Rating Scale for Depression (HAM-D). This finding is also consistent with the accepted etiology of 
chronic stages of pain and disability, where psychosocial symptoms are continuously exacerbated in a pain-stress cycle (Gatchel, 1996). However, all groups were comparable on the other psychosocial measures, contrary to the hypothesis that the ER group would demonstrate better overall responses on psychosocial measures at prerehabilitation. Although a trend towards poorer self-report of functional status as measured by the Million Visual Analog Scale was observed as duration of disability increased, only the DR group indicated significantly poorer scores with an effect size magnitude that was relatively negligible at 0.2 (a small effect, based on the criteria for Cohen's $d$ ).

The most noteworthy finding, however, was on health-related quality of life measured by the profile-based SF-36 and the corresponding utility-based scores derived from. Despite significantly increasing durations of disability across all three groups, pre-rehabilitation health-related quality of life was not significantly different among the three groups, with the utility-based quality of life virtually a constant for all groups. Although previous studies on this patient population have indicated moderate evidence for general health-related quality of life instruments, like the SF-36, in documenting treatment efficacy from pre-to-post rehabilitation (Gatchel, Mayer, Dersh, Robinson, \& Polatin, 1999; Wilson, 2007), the results of the present study indicate that such measures of general health status may not be sensitive enough to differentiate subgroups within the CDOMD population. In the context of the present study, it is likely that quality-of-life instruments may be not be adequately sensitive in discriminating 
among groups that have surpassed the timeline for chronic stages of pain and disability, i.e., greater than 4 months.

Besides the psychosocial variables discussed above, other pre-rehabilitation characteristics found to be significantly different among the groups may also play the role of psychosocial stressors. Specifically, the higher prevalence of attorney representation in the two late rehabilitation groups is an indicator of possible litigation within the medico-legal domain. While several previous studies have documented the role of litigation as a risk factor for poorer post-treatment outcomes like functional status and RTW (DeBerard, Masters, Colledge, Schleusener, \& Schlegel, 2001; Theodore, 2007; Wright, Mayer, \& Gatchel, 1999), a recent study on a cohort of patients with musculoskeletal injuries reported that ongoing litigation was also a strong predictor of poorer post-treatment psychosocial functioning (Bhandari et al., 2008).

The higher prevalence of surgeries in the late rehabilitation group also underscores an exacerbating factor for poorer psychosocial status. These late rehabilitation patients have arrived, or are close to arriving, at a terminal end-point for financial benefits related to their disability (Mayer \& Polatin, 2000), and are therefore still perpetuating in a pain-stress cycle with no remedy to severe functional deficits despite numerous surgical procedures. Given these significant exacerbations in psychosocial stressors for longer durations of disability, the overall results for prerehabilitation characteristics indicate a substantial benefit of early rehabilitation within the first $4-8$ months of persistent disability. 


\section{Treatment Efficacy}

Contrary to the two sets of hypotheses for patient characteristics following the functional restoration program, the data indicated that all three groups fared comparably on post-rehabilitation psychosocial measures, as well as on one-year objective, socioeconomic outcomes. Although an increasing linear trend of depressive symptoms was noted for increasing duration of disability, no overall significant group differences were observed. Consequent to this trend of increasing depressive symptoms, the DR group had almost twice as great a likelihood of having scores that ranged from moderate-to-severe depression. A significant linear trend for higher scores on perceived disability, within the range of extreme disability ( $\geq 101$ on the MVAS), was also observed as disability duration increased, however no significant overall differences were indicated for the groups. Consistent with findings at pre-rehabilitation, all groups fared equally well on pain intensity ratings, and general health status based on the SF36. Consequently, the corresponding utility-based quality of life measures derived from the SF-36 were also identical across all three groups.

At one-year post rehabilitation, the only significant finding was related to a linear trend for decreasing rates of work retention as a function of disability duration with no statistically significant differences among groups. All groups fared comparably, with no significant differences, on all the other objective socioeconomic outcomes as well. However, it should be noted that the outcome rates for the ER group and DR group were comparable to the outcome rates reported in an earlier study documenting the relationship between duration of disability and outcome rates (Jordan, Mayer, \& 
Gatchel, 1998). Although this previous study reported statistically significant differences between early and delayed rehabilitation, there are several underlying reasons for the non-significant findings in the present study.

A major difference between these two studies is the matched cohort design that removes any confounding effects for demographic variables like age and gender. Previous findings from this same patient population have indicated that both age and gender are significant predictors of poorer rates for occupational outcomes and increased healthcare utilization at one-year post-rehabilitation (Mayer, Gatchel, \& Evans, 2001; McGeary, Mayer, Gatchel, Anagnostis, \& Proctor, 2003; Theodore, 2007; Wilson, 2007). Specifically, a 4\% - 5\% reduction in the odds of RTW and work retention was observed for every one-year increase from the average age of the cohort. Similarly, female gender was uniquely associated with approximately 1.6 times greater odds of increased treatment-seeking during the one-year post-rehabilitation period. Given these previously reported associations between demographic factors and one-year outcome rates, the non-significant findings for one-year outcomes in the present study should not be surprising. Additionally, the comparable outcome rates across the three groups in this study are also substantiated by the documented strengths of the functional restoration program with regards to treatment efficacy and positive outcome rates, even when investigating sub-populations of patients with high-risk for poorer outcomes (Dersh et al., 2008; Dersh et al., 2007; Gatchel, Mayer, \& Theodore, 2006; Mayer et al., 1998; Mayer, Towns, Neblett, Theodore, \& Gatchel, 2008; Proctor, Mayer, Theodore, \& Gatchel, 2005; Theodore, 2007). 
In terms of source of disability income at one-year post-rehabilitation, both the ID group and DR group were more likely to be receiving Impairment Income Benefits (IIBS) and Supplemental Income Benefits (SIBS). The higher proportion of these payments in the two late rehabilitation groups are a likely result of higher impairment ratings determined prior to rehabilitation or at the end of the functional restoration program. Given the longer duration of disability, significant levels of physical deconditioning are expected with these patients (Mayer \& Press, 2005), thus resulting in higher impairment ratings that were still paying out as IIBS or SIBS at the one-year evaluation.

However, a greater proportion of cost-shifting out of the Workers' Compensation system and towards Federal disability benefits on SSDI, was observed for both the late rehabilitation groups. This is a noteworthy finding in light of the comparable case closure rates at one-year post-rehabilitation. As noted by Gatchel and Okifuji (2006), closure of disability claims have become an increasingly important outcome for interdisciplinary rehabilitation programs, given the economic implications of prolonged benefits payments. Although the vast majority of patients across the three groups $(97 \%$ - 99\%) had their Workers' Compensation claim closed, this high rate of case closure and the similarity of these closure rates across the groups masks the incurring of additional Federal indemnity costs that perpetuate outside the Workers' Compensation system. It should also be noted that Federal disability costs are not a stand-alone entity, but are likely accompanied by further productivity losses due to the 
injured worker not returning to an active occupational status (Gatchel \& Okifuji, 2006;

U. S. General Accounting Office, 2001).

\section{Cost-Effectiveness Analysis}

The cost-effectiveness analyses, using both natural outcomes (RTW and work retention) and quality of life scores, provided mixed results in terms of interpretability. Average cost-effectiveness ratios and their corresponding confidence intervals indicated that, overall, no significant differences could be concluded among the groups in the average cost per unit of outcome for RTW, work retention, as well as quality of life scores. However, given the statistically significant differences in costs within the analytic timeframe as well as slight variations on the objective outcomes across the three disability duration groups, the results indicated strong domination of the ID and DR groups by the ER group on the outcomes for RTW and work retention. This finding supports the hypothesis that early rehabilitation would be more cost-effectiveness relative to late rehabilitation. However, the identical quality of life scores across the three groups provided no opportunity to make meaningful inferences on costeffectiveness with respect to this outcome.

On the issue of negative incremental ratios, it should be noted that interpretation of negative incremental cost-effectiveness ratios is not possible, other than concluding dominance (Gift, Haddix, \& Corso, 2003; Gold, Siegel, Russell, \& Weinstein, 1996). Although in general, a negative ratio denotes cost-savings, it should also be noted that a larger magnitude of effectiveness in the denominator results in a smaller overall ratio. Assuming two separate ratios are negative, a larger magnitude of effectiveness in the 
denominator yields a smaller ratio compared to a competing ratio with a smaller magnitude of effectiveness in the denominator (Gift, Haddix, \& Corso, 2003). Going by magnitude of the ratio alone, the latter will be incorrectly concluded as being more costeffective given the larger savings. Therefore, interpretation of cost-savings in this regard is misleading. For this reason, negative incremental ratios are summarily regarded as indicating an unquestionably cost-effective alternative.

On a less positive note, the primary challenge with interpreting these results was the fact that all three groups fared comparably on all outcomes considered. Other than the case of a negative incremental ratio that denotes domination, significant differences in the outcomes are required to be able to interpret the incremental ratios and confidence intervals around this ratio (Heitjan, Moskowitz, \& Whang, 1999). Consequently, very small and non-significant differences in effectiveness lead to highly inflated values in the distribution of the incremental ratios. Furthermore, given the domination by the ER group and the comparable outcomes across all groups, the construction of cost-effectiveness acceptability curves will not aid in further interpretation, other than illustrating a constant straight line relationship.

As an alternative, incremental cost-effectiveness planes were constructed based on the joint distribution of incremental costs and outcomes observed from the bootstrapped estimates of each disability duration group. Following this, for each comparison corresponding to the incremental cost-effectiveness ratios, the probability of cost-savings and greater effectiveness was presented (Heitjan, 2008). As would be expected from the negative ratios, the ER group demonstrated a certainty for being cost- 
saving, relative to the ID and DR groups. However, in comparison to the ID group, a substantial proportion of the estimates indicated lower effectiveness. Similar to the comparison between the ER and DR groups, investigation of the joint distribution of incremental costs and effectiveness for the ID and DR groups indicated almost certain probabilities of greater effectiveness. But in contrast to the previous comparison, there was also a greater probability that rehabilitation during the intermediate period was more costly at the same time.

The present study is unable to establish a direct association between the patterns of distribution described above and other predictors or covariates of disability duration. This direct association is impossible simply because the above distributions are bootstrapped estimates based on the joint distribution of cost and effectiveness data for each disability duration group. However, the results do pave the way for new lines of inquiry on factors that impact the cost-savings for various sub-populations of patients (i.e., other patient groupings, in addition to disability duration). For example, the sequential regression analysis indicated several independent risk factors, unique from the effect of disability duration. Two of the biggest cost drivers within the analytical timeframe were prior surgical procedures and the diagnosis of Major Depressive Disorder (MDD). Additionally, a predictor of lower costs was having the Workers' Compensation case closed prior to admission in a functional restoration program.

While MDD is likely associated with increased psychosocial interventions throughout the phase of the program, patients with prior surgeries have a range of underlying psychosocial deficits, including higher rates of opioid dependence, reduced 
functional status, dependence on the disability benefit system, as well as significantly more medical complications related to the physical deconditioning syndrome (Chibnall, Tait, Andresen, \& Hadler, 2006; Dersh et al., 2008; Mannion \& Elfering, 2006; Theodore, 2007). On the other hand, the link between case closure and lower costs are possibly due to a reduction in secondary gain factors and medico-legal barriers to recovery that may require greater attention during the course of functional restoration (Dersh, Polatin, Leeman, \& Gatchel, 2004; Leeman, Polatin, Gatchel, \& Kishino, 2000). Future studies could investigate a broader group of predictors of costs, possibly stratified by specific sub-populations of interest, such as opioid dependent patients or those with specific low back surgeries. The identified differences in costs could then be readily linked to outcomes for a cost-effectiveness analysis, provided that relatively substantial and statistically significant differences are observed in outcome rates.

\section{Total Cost of Illness}

The present study is also the first, to date, in providing an estimate of the total cost of illness as a function of disability duration, in a CDOMD patient population that were referred to and completed a functional restoration program. The results indicated a staggering amount of costs accrued over the entire duration of disability, particularly for the DR group, with an estimated average cost per-patient of over $\$ 200,000$. Estimated average savings that could potentially be obtained through early rehabilitation for CDOMDs was $66 \%$, or approximately $\$ 140,000$, when considering patients with greater than 18 months of disability in the DR group. Savings of 39\%, or approximately $\$ 45,000$, was estimated when the ID group was compared to the ER group. The results 
further indicate that the greatest savings obtained through early rehabilitation of chronic disabled patients come from the costs accrued for disability benefits and lost productivity: $60 \%(\$ 11,082)$ and $61 \%(\$ 19,963)$, respectively, for the ID group; and, $80 \%(\$ 29,462)$ and $83 \%(\$ 60,528)$, respectively, for the DR group. Savings in medical costs were estimated at $68 \%(\$ 46,256)$ for the DR group and $37 \%(\$ 12,394)$, for the ID group.

Providing context for these figures is challenging, due to the fact that estimates of total cost of illness stratified by duration of disability are not available in the literature. Furthermore, cost estimates are often quoted as annual expenditure amounts. Some useful benchmarks, however, are available in the literature. For example, the average cost per claim (medical and disability benefits) in Texas for all injured workers' with lost time claims was \$14,323 during fiscal years 2005 - 2006 (Workers' Compensation Research Institute, 2007). In terms of total expenditure for chronic disability nationwide, medical costs for chronic pain nationwide were estimated at $\$ 70$ billion annually, with an additional $\$ 80$ billion in estimated costs of disability benefits (Gatchel \& Okifuji, 2006). Additionally, an estimated $\$ 61.2$ billion in annual costs of lost productivity was reported for musculoskeletal pain in general (Stewart, Ricci, Chee, Morganstein, \& Lipton, 2003).

Despite these staggering figures, context for the overall savings in lifetime medical costs per patient are promising, when intensive interdisciplinary rehabilitation is provided for the chronic pain patient. Lifetime medical costs for conventional, conservative treatment modalities are estimated to range from $\$ 412,800$ - $\$ 634,366$ per 
patient, whereas after interdisciplinary rehabilitation, lifetime medical costs are estimated to range from $\$ 140,190$ - $\$ 211,087$ per patient; providing a lifetime savings of approximately $67 \%$ (Gatchel \& Okifuji, 2006). The findings in the present study add further value to the literature on estimates of cost savings for interdisciplinary rehabilitation for chronic pain, by demonstrating that early rehabilitation itself can result in estimated savings of between $39 \%$ - $66 \%$ per patient, depending on how long the duration of disability extends.

\section{General Discussion}

Overall, the results indicated substantial benefit for early rehabilitation of chronic disability following a work-related injury. At the outset, the costs of medical care were estimated to be substantially lower for patients who were given an opportunity for intensive interdisciplinary rehabilitation within the first $4-8$ months of disability. Within this context, early rehabilitation was also associated with significantly lower rates of medical utilization, including surgical procedures both in terms of overall rates as well as in terms of the number of surgical procedures per patient. Numerous surgical procedures, especially for non-specific chronic low back pain, have been noted to be correlated with increased medical complications and greater susceptibility to psychosocial barriers to recovery (DeBerard, Masters, Colledge, Schleusener, \& Schlegel, 2001). Additionally, early rehabilitation also underscores prevention against development of complex psychosocial deficits, including depression, narcotic dependence, and a complex sequelae of comorbid psychopathology as denoted by the higher prevalence rates of combined Axis I and Axis II DSM-IV disorders in the late 
rehabilitation groups. Most notably, early rehabilitation substantially contains the costs of lost productivity and the accrual of disability benefits, both as a consequence of the shorter disability duration as well as the generally high rates of RTW following the functional restoration program.

In terms of outcomes following treatment, the efficacy of the functional restoration program results in comparable outcome rates independent of disability duration. These findings add to an ever-growing body of evidence that prevalent models for occupational disability, such as the Phase Model of Disability, need to be modified in terms of accounting for a greater role played by biopsychosocial factors in determining the etiology of disability. Duration of disability alone should not be the central factor in articulating the outcomes and potential recovery for the injured worker. In this regard, it is the significant role of early predictors and risk-factors of long-term disability that should be the major focus of models of occupational disability, including the psychosocial and socioeconomic factors that have been consistently linked to poorer outcomes (Gatchel et al., 2003; Turner et al., 2008).

The conventional index of effectiveness, based on quality-of-life measures, did not add anything substantial to the results obtained in the present study. Although the strictest definition of a quality-of-life measure, i.e. the Quality-Adjusted Life Year (QALY), was not tested in the present study, there are some well-founded reservations against using general health status and quality of life outcomes in a Workers' Compensation population. Specifically, a recent study from this same treatment program has demonstrated that changes in self-report measure of general health status, 
i.e. the SF-36, has very little association with objective indicators of treatment outcome relevant to the Workers' Compensation population, such as occupational status and healthcare utilization (Wilson, 2007). In the present study, utilizing the SF-36 to derive utility-based quality-of-life measures would no doubt be subject to the same limitations previously reported. However, the lack of significant differences among the groups on all measures of outcome prevents any further tests on the relationship between qualityof-life and objective outcomes. Despite these limitations, the results utilizing objective outcomes (RTW and work retention) provided support for the hypothesis on the greater cost-effectiveness of early rehabilitation. Considering that the Workers' Compensation system and occupational disability, in general, provide an opportunity for measuring objective outcomes directly linked to resource use, greater emphasis should be placed on outcomes such as occupational status and healthcare resource use. In this regard, early work by Loisel, et al. (2002) has explored the use of the number of days disabled as the effectiveness component in comparing several treatment options as part of a costeffectiveness analysis. Given that the various stakeholders and decision makers within the Workers' Compensation setting emphasize the importance of resuming an active occupational status, a re-definition of effectiveness measures unique to the Workers' Compensation setting should be formulated as part of the theoretical framework addressing the application of economic evaluation methods to issues related to occupational disability. 


\section{Limitations of the Present Study}

A large study like the present one is rarely accomplished without some limitations. A significant contributor to the limitation of this study was the fact that all data associated with medical utilization were retrospective in nature, and not reliably collected in a manner that would enable estimation of costs. To overcome this, a comparable cohort of Workers' Compensation patients from a different State jurisdiction was used to estimate medical costs. It should be noted, that although average length of disability, distribution of patients within the disability groups, gender, and age were not exactly similar between the PRIDE and OBWC cohorts, these differences pose no barriers for the purposes of extracting cost estimates. There are several reasons for this. Firstly, similarities between the two cohorts would be important if the objective was to compare them, either on outcomes or some other characteristics, but this is not the case in the present study. Secondly, although average length of disability differs between the two cohorts, especially on the extended disability group, it should be noted that the range of disability duration within each group is similar. Thirdly, the effect of gender and age on total medical costs in the OBWC cohort was negligible. Although comparable in terms of range of disability duration, injured musculoskeletal regions, and prevalence of surgeries, it should be emphasized that the Workers' Compensation system in Texas is known for high medical utilization rates, relative to other State Workers' Compensation jurisdiction (Eccleston \& Zhao, 2005). Since the vast majority of patients in the present study were within the Texas Workers' 
Compensation system, medical cost estimates in the present study should be considered a lower limit of what the actual costs may be in reality.

A second limitation is associated with the estimates of disability benefits. Although the initial proposal elaborated on a method to estimate disability payments from Social Security Disability Insurance (SSDI), estimating the cost of SSDI across the duration of disability was not possible with estimated weekly or monthly payments alone, and absent a payment start date or duration of time for the payments.

Compounding this problem further is that a weekly or monthly SSDI payment estimated from the Social Security Administration may not be reflective of the true payments disbursed, since SSDI payment amounts are reduced when patients are receiving disability benefits from the Workers' Compensation system (Social Security Administration, 2008). Another source of limitation in the estimate of disability benefits is the exclusion of Supplemental Income Benefits (SIBS) paid out within the Texas Workers' Compensation jurisdiction. The jurisdictional rules that determine eligibility for SIBS payments are too complex to warrant estimation without knowing two key pieces of information: i.e., the number of quarters in a year that patients qualified for SIBS payments; and, the average weekly wage for any RTW that is less than $80 \%$ of pre-injury average weekly wage. The full details on how SIBS benefits are determined are provided in Appendix E. Finally, a small percentage of patients received private disability benefits in the form of Short-Term Disability (STD) and Long-Term Disability (LTD) payments. Both types of payments were not included in the total costs for disability benefits due to uncertain parameters required for estimating payments. For 
example, STD and LTD payments are dependent on the type of insurance policy the patient is covered by, resulting in varying ranges for compensation $(50 \%-100 \%$ of wages) and limits on the duration of payments ( $9-52$ weeks for STD; 2 years retirement for LTD). Additionally, like SSDI payments, the STD and LTD payments are affected by the amount of other compensation received from State or Federal sources. Therefore, estimation is neither feasible nor possible for the small amount of patients in the cohort $(N=69)$ who were identified as having received these benefits specified above.

A third limitation is in the timeframe that was used to measure changes in health-related quality of life. Although it is common for health-related quality of life measures to span less than one-year post-treatment in CDOMD studies (Hatten, Gatchel, Polatin, \& Stowell, 2006; Vetter, 2007), to adhere to the strictest definition of a Quality-Adjusted Life Year (QALY) requires health-related quality of life measures collected at one-year post-treatment. The initial proposal specified a means of estimating one-year post-treatment quality of life measures for the three disability duration groups from a separate cohort of CDOMD patients receiving interdisciplinary rehabilitation at the Eugene McDermott Center for Pain Management at the University of Texas Southwestern campus. However, upon evaluation of this external cohort, it was discovered that the one-year SF-36 quality of life scores as well as the derived utility-based quality of life scores were approximately identical to the post-treatment scores observed in the PRIDE cohort (see Appendix F). In addition, neither the SF-36 scores nor the utility-based quality of life scores significantly differed among the three 
disability duration groups in this external cohort. The small sample size of this cohort should also serve as a caution against using these scores as direct estimates. Therefore, utilizing this external cohort adds no value to the present study while perpetuating the similar challenges on negligible effectiveness measures across the groups. Furthermore, another challenge is prominent when using an external estimate of outcomes. Any interpretation of cost-effectiveness would require confidence intervals as well as incremental cost-effectiveness planes. However, these can only be achieved by generating a distribution of bootstrapped estimates based on the joint distribution of costs and outcomes. Any measure external to the PRIDE cohort immediately negates this possibility.

A fourth potential limitation of this study is that the cohort consists of only program completers. Additionally, program completion rate was significantly different for the DR group, with a greater number of dropouts $(\sim 8 \%)$, relative to the ER group. Such an exclusion criterion may be criticized for introducing a selection bias in the study. However, inclusion of non-completers is not possible for three main reasons. Firstly, non-completers do not have any post-rehabilitation measures, including the SF36 , and will therefore not be included in the final cost-effectiveness analyses due to the inability to estimate post-treatment quality-of-life scores. Secondly, one-year socioeconomic outcomes are available only on a limited number of non-completers, at a contact rate of approximately $50 \%$. This will further complicate cost-effectiveness calculations utilizing natural-unit outcomes as well as one-year cost estimates based on some of the one-year outcomes, like visits to healthcare providers and new surgeries. 
Finally, program non-completers also constitute a separate population of patients that present at pre-rehabilitation with a unique set of psychosocial barriers to recovery that differentiate them from program completers. These different characteristics include elevated rates of Axis II DSM-IV disorders, increased medico-legal barriers to recovery, and significant amounts of secondary gain issues related to relationship with the employer (Dersh et al., 2007; Howard, Mayer, Theodore, Shea, \& Gatchel, in press; Proctor, Mayer, Theodore, \& Gatchel, 2005). Given these issues, the exclusion of noncompleters was justified on the basis of avoiding significant missing data problems that would have resulted in distorted summary statistics, as well as avoiding patient selection factors that result in an unrepresentative sub-population of CDOMD patients being included in the study.

Finally, the present study was not a randomized controlled trial (RCT), thus preventing any inferences of causality. Given ethical considerations and legal requirements with regard to providing the best available care to all patients, it is therefore unrealistic to expect RCT designs for research purposes within this area of investigation. Furthermore, even at the outset of injury, it will be impossible to a priori randomly assign patients into disability duration groups. Nevertheless, randomized trials have been utilized in evaluating functional restoration within nations adopting socialized medicine, and the results have documented the superiority of the functional restoration approach in rehabilitating CDOMD patients relative to less intensive singlediscipline treatment modalities (Bendix et al., 1996; Corey, Koepfler, Etlin, \& Day, 1996; Hildebrandt, Pfingsten, Saur, \& Jansen, 1997; Jousset et al., 2004). Given the 
logistical limitations, a prospective matched cohort design was best suited to explore the research question of interest in this study, i.e., the effect of disability duration on both the treatment- and cost-effectiveness of a functional restoration program. Prospective cohort designs define a grouping variable of interest (i.e., disability duration groups) prior to the undertaking of the study. Additionally, data are collected in a prospective manner over time (i.e., one-year outcome variables), eliminating some of the more critical biases associated with non-RCT designs, such as using data from convenience sampling. Finally, in terms of internationally-accepted standards for levels of evidence established by the Oxford Center for Evidence-Based Medicine, prospective cohort designs rank $2^{\text {nd }}$ in terms of quality after RCT designs.

\section{Conclusion}

The present study provides three main findings. Firstly, duration of disability is not a significant contributing factor to poorer outcomes, when intensive interdisciplinary rehabilitation, such as functional restoration, is provided for chronic disabling occupational musculoskeletal disorders. Secondly, the timeliness of providing a course of functional restoration, between $4-8$ months post-injury, is a cost-effective alternative to conventional practices of administering multiple, single-modality treatments. Thirdly, when the costs incurred over the duration of disability are accounted for, cost-savings of between $39 \%$ - $66 \%$ can be potentially achieved with early rehabilitation. The results of the study are also timely, given an increasing trend of engaging in treatment "carve-out" practices by the healthcare financing sector (Gatchel \& Okifuji, 2006). This practice prevents patients from accessing interdisciplinary 
treatments that have been proven successful at alleviating and/or managing pain and disability. As a result, many patients may progress into late chronic stages of disability, incur considerable costs by trying many types of conservative care modalities or surgeries, and may finally receive intensive interdisciplinary treatment as a "last resort" despite the fact that early rehabilitation with this treatment modality would have been the most cost-effective strategy of treatment. Finally, the results document the heuristic value inherent in the biopsychosocial paradigm of managing pain and disability, and add to a growing body of evidence documenting the potential for significant costsavings, in addition to greater treatment efficacy associated with treatment modalities based on the biopsychosocial model of pain. 
APPENDIX A

TABLES 
Table A.1. Definition of One-Year Socioeconomic Outcome Measures

\begin{tabular}{|l|l|}
\hline Socioeconomic Outcomes & Definition \\
\hline Return to work & $\begin{array}{l}\text { Any reported period of work during the one } \\
\text { year post-rehabilitation }\end{array}$ \\
\hline Work Retention & $\begin{array}{l}\text { Maintaining employment during the time of } \\
\text { the one-year interview }\end{array}$ \\
\hline $\begin{array}{l}\text { Seeking Treatment from a New Healthcare } \\
\text { Provider }\end{array}$ & $\begin{array}{l}\text { Percentage of patients seeking additional } \\
\text { healthcare from healthcare providers other } \\
\text { than the treating or rehabilitation physicians } \\
\text { during the post-rehabilitation year }\end{array}$ \\
\hline Mean Visits to New Healthcare Provider & $\begin{array}{l}\text { Number of visits to providers other than the } \\
\text { treating or rehabilitation physicians during } \\
\text { the post-rehabilitation year }\end{array}$ \\
\hline $\begin{array}{l}\text { New spine surgeries to originally injured } \\
\text { region }\end{array}$ & $\begin{array}{l}\text { New surgeries to the compensable injured } \\
\text { region anytime during the post-rehabilitation } \\
\text { year }\end{array}$ \\
\hline New injuries to originally injured region & $\begin{array}{l}\text { Recurrent injuries to the original region } \\
\text { resulting in lost time from work }\end{array}$ \\
\hline Worker's compensation case settlement & $\begin{array}{l}\text { Ongoing financial disputes or litigation } \\
\text { related to the injury }\end{array}$ \\
\hline Disability Income Source & $\begin{array}{l}\text { Sources of disability income based on the } \\
\text { following categories: TTD benefits; } \\
\text { impairment benefits, private disability } \\
\text { benefits; Federal Disability benefits } \\
\text { (SSDI/SSI); and Supplemental Income } \\
\text { Benefits. }\end{array}$ \\
\hline
\end{tabular}


Table A.2. Cost Inventory

\begin{tabular}{|c|c|}
\hline Type of Cost & Source of Data \\
\hline $\begin{aligned} & \text { Pre-Rehabilitation Medical Costs } \\
& \text { - } \text { Surgeries } \\
& \text { - } \text { Ancillary costs of surgery } \\
& \text { - } \text { Diagnostics } \\
& \text { - } \text { Injection therapeutics } \\
& \text { - } \text { Evaluation \& Management } \\
& \text { - } \text { Hospital costs } \\
& \text { - } \text { Non-surgical treatment } \\
& \text { modalities } \\
& \text { - } \text { Medications }\end{aligned}$ & $\begin{array}{l}\text { All medical costs estimated from Ohio's } \\
\text { BWC }\end{array}$ \\
\hline 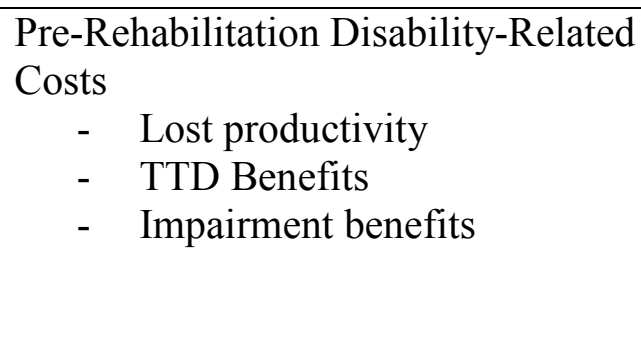 & $\begin{array}{l}\text { All disability-related costs estimated using } \\
\text { data from the PRIDE Electronic Research } \\
\text { Database, PRIDE Research Folders, and } \\
\text { regulations by the relevant Workers' } \\
\text { Compensation jurisdictions }\end{array}$ \\
\hline $\begin{aligned} & \text { Rehabilitation Program Costs } \\
& \text { - } \text { Billed treatment hours } \\
& \text { - } \text { PRIDE doctor's visits } \\
& \text { - } \text { PRIDE injection therapeutics } \\
& \text { - } \text { Medications }\end{aligned}$ & $\begin{array}{l}\text { PRIDE research database } \\
\text { Epocrates, Inc. }\end{array}$ \\
\hline $\begin{array}{l}\text { One-Year Costs } \\
\text { - } \quad \text { Surgeries \& Related Costs } \\
\text { - } \quad \text { Visits to New Healthcare } \\
\\
\quad \text { Providers }\end{array}$ & $\begin{array}{l}\text { Center for Medicare and Medicaid Services' } \\
\text { U.S. Agency for Healthcare Research and } \\
\text { Quality }\end{array}$ \\
\hline
\end{tabular}


Table A.3. Estimated Total Costs of Specific Musculoskeletal Surgeries based on Medicare Reimbursements

\begin{tabular}{|l|c|}
\hline Type of Surgery & Cost per Surgery \\
\hline $\begin{array}{l}\text { Major Arm and Shoulder Surgeries - with complications or } \\
\text { pre-existing conditions }\end{array}$ & $\$ 7,182$ \\
\hline Two or more Hip, Knee, or Ankle Surgeries & $\$ 19,418$ \\
\hline $\begin{array}{l}\text { Replacement of Hip, Knee, or Ankle or Reattachment of } \\
\text { Thigh, Foot, or Ankle }\end{array}$ & $\$ 11,916$ \\
\hline Repair of Previous Hip or Knee Replacement & $\$ 15,552$ \\
\hline $\begin{array}{l}\text { Lumbar Fusion - with Complications or Pre-existing } \\
\text { Conditions }\end{array}$ & $\$ 23,555$ \\
\hline Lumbar Fusion & $\$ 18,094$ \\
\hline $\begin{array}{l}\text { Cervical Fusion - with Complications or Pre-existing } \\
\text { Conditions }\end{array}$ & $\$ 16,706$ \\
\hline Cervical Fusion & $\$ 8,786$ \\
\hline $\begin{array}{l}\text { Spine Surgeries (excl. fusion) - with complications or pre- } \\
\text { existing conditions }\end{array}$ & $\$ 5,708$ \\
\hline Spine Surgeries (excl. fusion) & \\
\hline
\end{tabular}


Table A.4. Six-variable Regression Model to be Used as the Algorithm to Convert SF36 Scale Scores into Utility-Based QWB Scores

\begin{tabular}{|l|r|}
\hline Variable & Coefficient \\
\hline Coefficient & 0.59196 \\
SF-36 Scales & \\
Physical Function & 0.0012588 \\
Mental Health & -0.0011709 \\
Bodily Pain & -0.0014261 \\
(General Health Perceptions) x (Role Function: physical) & 0.00000705 \\
(Physical Function) x (Bodily Pain) & 0.00001140 \\
(Mental Health) x (Bodily Pain) & 0.00001931 \\
\hline
\end{tabular}


Table A.5. Program Completion Rates across Disability Duration Groups $(\mathrm{N}=2454)$

\begin{tabular}{|c|c|c|c|c|c|}
\hline Variables & $\begin{array}{c}\text { ER } \\
\mathrm{N}=859\end{array}$ & $\begin{array}{c}\text { ID } \\
\mathrm{N}=788\end{array}$ & $\begin{array}{c}\text { DR } \\
\mathrm{N}=807\end{array}$ & $\begin{array}{c}\text { p-values } \\
\text { group diff/lin. trend }\end{array}$ & $\begin{array}{c}\text { Effect Size } \\
w\end{array}$ \\
\hline Program Completion [\% (n)] & $79.5(683)$ & $77.2(608)$ & $71.1(574)^{*}$ & $<.001 /<.001$ & .083 \\
\hline
\end{tabular}

* Significantly different compared to ER group, at $\mathrm{p}<.05$ after adjusting for inflation of Type I error

Table A.6. Effect Sizes for Post-Hoc Comparisons of Program Completion ( $\mathrm{N}=2454)$

\begin{tabular}{|l|c|c|}
\hline \multirow{2}{*}{ Variables } & \multicolumn{2}{|c|}{ Odds Ratio (95\% CI) } \\
\cline { 2 - 3 } & ID vs. ER & DR vs. ER \\
\hline Program Completion & NS & $0.6(0.5,0.8)$ \\
\hline
\end{tabular}


Table A.7. Demographic Characteristics of the Cohort $(\mathrm{N}=1119)$

\begin{tabular}{|c|c|c|c|c|}
\hline Variables & $\begin{array}{c}\text { ER } \\
N=373\end{array}$ & $\begin{array}{c}\text { ID } \\
\mathrm{N}=373\end{array}$ & $\begin{array}{c}\text { DR } \\
N=373\end{array}$ & $\begin{array}{c}\text { p-values } \\
\text { group diff/lin. trend }\end{array}$ \\
\hline Age (SD) & $44.7(8.7)$ & $45.1(8.6)$ & $45.5(8.3)$ & $.396 / .174$ \\
\hline $\begin{array}{l}\text { Age Groups }[\%(\mathrm{n})] \\
\quad \leq 30 \\
\quad 31-40 \\
41-50 \\
51-60 \\
>60\end{array}$ & $\begin{array}{c}1.9(7) \\
25.2(94) \\
43.4(162) \\
26.3(98) \\
3.2(12)\end{array}$ & $\begin{array}{c}1.9(7) \\
25.2(94) \\
43.4(162) \\
26.3(98) \\
3.2(12)\end{array}$ & $\begin{array}{c}1.9(7) \\
25.2(94) \\
43.4(162) \\
26.3(98) \\
3.2(12)\end{array}$ & $1.000 / 1.000$ \\
\hline Gender $[\%$ Male $(\mathrm{n})]$ & $53.1(198)$ & $53.1(198)$ & $53.1(198)$ & $1.000 / 1.000$ \\
\hline $\begin{array}{l}\text { Ethnicity }[\%(\mathrm{n})] \\
\text { Caucasian } \\
\text { African-American } \\
\text { Hispanic }\end{array}$ & $\begin{array}{l}57.9(216) \\
21.2(79) \\
20.9(78)\end{array}$ & $\begin{array}{l}57.9(216) \\
21.2(79) \\
20.9(78)\end{array}$ & $\begin{array}{l}57.9(216) \\
21.2(79) \\
20.9(78)\end{array}$ & $1.000 / 1.000$ \\
\hline $\begin{array}{l}\text { Injured Musculoskeletal Regions [\% (n)] } \\
\text { Cervical only } \\
\text { Lumbar/Thoracic only } \\
\text { Multiple Spinal } \\
\text { Upper/Lower Extremities only } \\
\text { Multiple Musculoskeletal (at least } 1 \text { spinal) }\end{array}$ & $\begin{array}{c}2.1(8) \\
43.4(162) \\
5.6(21) \\
24.9(93) \\
23.9(89)\end{array}$ & $\begin{array}{c}2.1(8) \\
43.4(162) \\
5.6(21) \\
24.9(93) \\
23.9(89)\end{array}$ & $\begin{array}{c}2.1(8) \\
43.4(162) \\
5.6(21) \\
24.9(93) \\
23.9(89)\end{array}$ & 1.000 \\
\hline
\end{tabular}


Table A.7 - Continued

\begin{tabular}{|c|c|c|c|c|}
\hline $\begin{array}{l}\text { Marital Status [\% (n)] } \\
\text { Single } \\
\text { Married/Significant Other } \\
\text { Separated/Divorced } \\
\text { Widowed }\end{array}$ & $\begin{array}{c}12.3(38) \\
61.2(189) \\
24.3(75) \\
2.3(7)\end{array}$ & $\begin{array}{c}12.2(38) \\
61.5(192) \\
24.7(77) \\
1.6(5)\end{array}$ & $\begin{array}{c}10.6(31) \\
59.4(174) \\
28.3(83) \\
1.7(5)\end{array}$ & $.902 / .383$ \\
\hline Level of Education in years (SD) & $11.7(2.9)$ & $11.5(2.8)$ & $11.5(3.1)$ & $.653 / .366$ \\
\hline $\begin{array}{l}\text { Length of Disability in months (SD) } \\
\text { Log Length of Disability (SD) }\end{array}$ & $\begin{array}{l}5.4(1.5) \\
1.7(0.3)\end{array}$ & $\begin{array}{l}13.0(2.8) \\
2.5(0.2)^{*}\end{array}$ & $\begin{array}{c}37.5(28.0) \\
3.5(0.5)^{*}\end{array}$ & $<.001 /<.001$ \\
\hline
\end{tabular}

* Significantly different compared to ER group, at $\mathrm{p}<.05$ after adjusting for inflation of Type I error 
Table A.8. Pre-Rehabilitation Utilization Rates for Medical Care $(\mathrm{N}=1119)$

\begin{tabular}{|c|c|c|c|c|c|}
\hline Variables & $\begin{array}{c}\text { ER } \\
N=373\end{array}$ & $\begin{array}{c}\text { ID } \\
N=373\end{array}$ & $\begin{array}{c}\text { DR } \\
N=373\end{array}$ & $\begin{array}{c}\mathrm{p} \text {-values } \\
\text { group diff/lin. trend }\end{array}$ & $\begin{array}{l}\text { Effect Size } \\
\eta^{2} \text { or } w\end{array}$ \\
\hline Surgeries $[\%(n)]$ & $16.8(62)$ & $47.2(174)^{*}$ & $66.5(244)^{*}$ & $<.001 /<.001$ & .413 \\
\hline $\begin{array}{l}\text { Number of Surgeries } \\
\text { All Patients, Mean (SD) } \\
\text { Surgical Patients, Mean (SD) } \\
\text { 1 Surgery only [\% (n)] }\end{array}$ & $\begin{array}{l}0.2(0.6) \\
1.3(0.8) \\
82.3(51)\end{array}$ & $\begin{array}{l}0.7(1.1)^{*} \\
1.5(1.1) \\
67.2(117)\end{array}$ & $\begin{array}{c}1.2(1.2)^{*} \\
1.8(1.1)^{*} \\
51.2(125)^{*}\end{array}$ & $\begin{array}{l}<.001 /<.001 \\
.001 / .001 \\
<.001 /<.001\end{array}$ & $\begin{array}{l}.141 \\
.030 \\
.226\end{array}$ \\
\hline $\begin{array}{l}\text { Type of Surgery }[\%(\mathrm{n})] \\
\text { Spine Surgeries } \\
\text { Other Musculoskeletal Surgeries } \\
\text { Unspecified Procedures }\end{array}$ & $\begin{array}{c}5.7(21) \\
10.8(40) \\
1.4(5)\end{array}$ & $\begin{array}{c}20.9(77)^{*} \\
24.7(91)^{*} \\
3.3(12)\end{array}$ & $\begin{array}{c}37.1(136)^{*} \\
30.5(112)^{*} \\
6.0(22)^{*}\end{array}$ & $\begin{array}{l}<.001 /<.001 \\
<.001 /<.001 \\
.003 / .001\end{array}$ & $\begin{array}{l}.314 \\
.200 \\
.103\end{array}$ \\
\hline $\begin{array}{l}\text { Diagnostics [\% }(\mathrm{n})] \\
\text { No Diagnostics } \\
\text { None Postoperative } \\
\text { X-Rays } \\
\text { Magnetic Resonance Imaging } \\
\text { EMG/Nerve Conduction Study } \\
\text { Other Diagnostics }\end{array}$ & $\begin{array}{c}0.3(1) \\
2.8(9) \\
72.3(235) \\
80.0(260) \\
47.4(154) \\
24.3(79)\end{array}$ & $\begin{array}{c}0.6(2) \\
7.7(26) \\
63.6(215) \\
68.6(232) \\
45.9(155) \\
29.0(98)\end{array}$ & $\begin{array}{c}0.9(3) \\
8.5(28) \\
59.6(196) \\
58.7(193) \\
34.7(114) \\
31.6(104)\end{array}$ & N/A & \\
\hline
\end{tabular}


Table A.8 - Continued

\begin{tabular}{|c|c|c|c|c|}
\hline $\begin{array}{l}\text { Therapeutic Injections [\% (n)] } \\
\text { None/None Postoperative } \\
\text { Epidural Steroid Injections } \\
\text { Facet Injections } \\
\text { Sacroilliac Joint Injections } \\
\text { Trigger Point Injections } \\
\text { Other }\end{array}$ & $\begin{array}{l}47.7(155) \\
24.9(81) \\
19.7(64) \\
8.3(27) \\
3.1(10) \\
15.4(50)\end{array}$ & $\begin{array}{l}40.5(137) \\
35.2(119) \\
25.1(85) \\
10.1(34) \\
4.4(15) \\
19.8(67)\end{array}$ & $\begin{array}{l}44.7(147) \\
26.4(87) \\
23.7(78) \\
9.4(31) \\
4.6(15) \\
18.2(60)\end{array}$ & $\mathrm{N} / \mathrm{A}$ \\
\hline $\begin{array}{l}\text { Primary/Secondary Care Modalities } \\
\%(\mathrm{n}) \\
\text { Visits (SD) }\end{array}$ & $\begin{array}{c}88.8(229) \\
30.6(24.2)\end{array}$ & $\begin{array}{l}91.1(246) \\
44.2(38.3)\end{array}$ & $\begin{array}{l}91.8(234) \\
47.1(42.2)\end{array}$ & $\mathrm{N} / \mathrm{A}$ \\
\hline $\begin{array}{l}\text { Work Hardening/Conditioning } \\
\% \text { (n) } \\
\text { Visits (SD) }\end{array}$ & $\begin{array}{c}16.7(43) \\
23.7(13.8)\end{array}$ & $\begin{array}{c}24.4(66) \\
22.6(13.0)\end{array}$ & $\begin{array}{c}26.3(67) \\
24.3(13.8)\end{array}$ & $\mathrm{N} / \mathrm{A}$ \\
\hline $\begin{array}{l}\text { Chiropractic Manipulation } \\
\%(\mathrm{n}) \\
\text { Visits (SD) }\end{array}$ & $\begin{array}{c}11.6(30) \\
\text { N/A }\end{array}$ & $\begin{array}{c}11.9(32) \\
\text { N/A }\end{array}$ & $\begin{array}{c}8.2(21) \\
\mathrm{N} / \mathrm{A}\end{array}$ & $\mathrm{N} / \mathrm{A}$ \\
\hline $\begin{array}{l}\text { Pain Management } \\
\% \text { (n) } \\
\text { Visits (SD) }\end{array}$ & $\begin{array}{c}0.4(1) \\
\text { N/A }\end{array}$ & $\begin{array}{c}3.3(9) \\
\text { N/A }\end{array}$ & $\begin{array}{c}6.7(17) \\
\mathrm{N} / \mathrm{A}\end{array}$ & $\mathrm{N} / \mathrm{A}$ \\
\hline
\end{tabular}

* Significantly different compared to ER group, at $p<.05$ after adjusting for inflation of Type I error 
Table A.9. Effect Sizes for Post-Hoc Comparisons for Significant Differences in Medical Utilization $(\mathrm{N}=1119)$

\begin{tabular}{|l|c|c|}
\hline \multirow{2}{*}{ Variables } & \multicolumn{2}{|c|}{ Cohen's $d$ or Odds Ratio (95\% CI) } \\
\cline { 2 - 3 } & ER vs. ID & ER vs. DR \\
\hline Surgeries [\% (n)] & $4.4(3.2,6.2)$ & $9.9(7.0,14.0)$ \\
\hline Number of Surgeries & & \\
All Patients, Mean (SD) & 0.5 & 1.0 \\
Surgical Patients, Mean (SD) & NS & 0.5 \\
1 Surgery [\% (n)] & $2.9(2.0,4.2)$ & $3.2(2.2,4.7)$ \\
2 Surgeries [\% (n)] & $6.5(2.9,14.6)$ & $11.6(5.2,25.6)$ \\
3 or more [\% (n)] & $4.1(1.4,12.5)$ & $15.1(5.4,42.2)$ \\
& & $9.8(6.0,16.0)$ \\
\hline Type of Surgery [\% (n)] & $4.4(2.6,7.3)$ & $3.6(2.4,5.4)$ \\
Spine Surgeries & $2.7(1.8,4.0)$ & $3.9(1.9,8.3)$ \\
Other Musculoskeletal Surgeries & NS & \\
Unspecified Procedures & \multicolumn{2}{|c}{} \\
\hline \multicolumn{2}{|c|}{} & \\
\hline
\end{tabular}


Table A.10. Occupational and Disability-related Characteristics of Cohort $(\mathrm{N}=1119)$

\begin{tabular}{|c|c|c|c|c|c|}
\hline Variables & $\begin{array}{c}\text { ER } \\
N=373\end{array}$ & $\begin{array}{c}\text { ID } \\
N=373\end{array}$ & $\begin{array}{c}\text { DR } \\
N=373\end{array}$ & $\begin{array}{c}\mathrm{p} \text {-values } \\
\text { group diff/lin. trend }\end{array}$ & $\begin{array}{l}\text { Effect Size } \\
\eta^{2} \text { or } w\end{array}$ \\
\hline $\begin{array}{l}\text { Occupational Category [\% (n)] } \\
\text { Professional,Technical,Managerial } \\
\text { Clerical \& Sales } \\
\text { Service Industry } \\
\text { Agriculture } \\
\text { Chemical \& Refining } \\
\text { Machine Trade } \\
\text { Light Manufacturing } \\
\text { Construction Trades } \\
\text { Miscellaneous }\end{array}$ & $\begin{array}{l}11.7(43) \\
16.8(62) \\
13.6(50) \\
0.5(2) \\
0.3(1) \\
4.3(16) \\
10.9(40) \\
7.9(29) \\
34.0(125)\end{array}$ & $\begin{array}{l}16.5(60) \\
15.4(56) \\
13.5(49) \\
0.3(1) \\
0.3(1) \\
6.3(23) \\
9.3(34) \\
9.6(35) \\
28.8(105)\end{array}$ & $\begin{array}{l}13.5(49) \\
15.7(57) \\
15.5(56) \\
0.8(3) \\
0 \\
2.8(10) \\
12.4(45) \\
14.1(51) \\
25.1(91)\end{array}$ & .074 & \\
\hline $\begin{array}{l}\text { Job Demand [\% (n)] } \\
\text { Sedentary-Light } \\
\text { Light-Medium } \\
\text { Medium-Heavy } \\
\text { Heavy-Very Heavy }\end{array}$ & $\begin{array}{c}14.6(54) \\
25.9(96) \\
32.4(120) \\
27.0(100)\end{array}$ & $\begin{array}{c}14.1(52) \\
25.4(94) \\
33.0(122) \\
27.6(102)\end{array}$ & $\begin{array}{c}12.8(47) \\
25.6(94) \\
37.6(138) \\
24.0(88)\end{array}$ & .776 & \\
\hline Pre-Injury Weekly Wage (SD) & $\$ 509(\$ 252)$ & $\$ 544(\$ 355)$ & $\$ 539(\$ 291)$ & $.237 / .177$ & \\
\hline Working at Admission [\% (n)] & $14.9(55)$ & $11.3(41)$ & $16.1(59)$ & $.150 / .650$ & \\
\hline Original Job Available [\% (n)] & $66.5(244)$ & $47.1(172)^{*}$ & $34.4(125)^{*}$ & $<.001 /<.001$ & .264 \\
\hline $\begin{array}{l}\text { TTD Months (SD) } \\
\text { Log Months (SD) }\end{array}$ & $\begin{array}{l}3.8(2.3) \\
1.3(0.6)\end{array}$ & $\begin{array}{l}10.0(4.7) \\
2.2(0.6)^{*}\end{array}$ & $\begin{array}{l}23.8(18.4) \\
2.9(0.8)^{*}\end{array}$ & $<.001 /<.001$ & .495 \\
\hline
\end{tabular}


Table A.10 - Continued

\begin{tabular}{|l|c|c|c|c|c|}
\hline Texas Workers' Compensation [\% (n)] & $91.7(342)$ & $93.6(373)$ & $89.5(334)$ & $.140 / .291$ & \\
\hline Case Settled [\% (n)] & $7.8(29)$ & $20.2(75)^{*}$ & $54.3(200)^{*}$ & $<.001 /<.001$ & .440 \\
\hline Receiving SSDI/SSI [\% (n)] & $0.3(1)$ & $0.3(1)$ & $5.6(22)^{*}$ & $<.001 /<.001$ & .178 \\
\hline Attorney Retained [\% (n)] & $11.8(43)$ & $24.7(91)^{*}$ & $25.5(92)^{*}$ & $<.001 /<.001$ & .154 \\
\hline
\end{tabular}

* Significantly different compared to ER group, at $p<.05$ after adjusting for inflation of Type I error 
Table A.11. Effect Sizes for Post-Hoc Comparisons of Significant Occupational and Disability-related Variables $(\mathrm{N}=1119)$

\begin{tabular}{|l|c|c|}
\hline \multirow{2}{*}{ Variables } & \multicolumn{2}{|c|}{ Cohen's $d$ or Odds Ratio (95\% CI) } \\
\cline { 2 - 3 } & ID vs. ER & DR. vs. ER \\
\hline Original Job Available & $0.4(0.3,0.6)$ & $0.3(0.2,0.4)$ \\
\hline TTD Months & 1.4 & 2.4 \\
\hline Case Settled & $3.0(1.9,4.7)$ & $14.0(9.1,21.5)$ \\
\hline Receiving SSDI/SSI & NS & $22.2(3.0,165.9)$ \\
\hline Attorney Retained & $2.4(1.6,3.6)$ & $2.6(1.7,3.8)$ \\
\hline
\end{tabular}


Table A.12. Axis I and Axis II DSM-IV Psychiatric Diagnoses at Admission ( $\mathrm{N}=1119)$

\begin{tabular}{|c|c|c|c|c|c|}
\hline Variables & $\begin{array}{c}\text { ER } \\
N=373\end{array}$ & $\begin{array}{c}\text { ID } \\
N=373\end{array}$ & $\begin{array}{c}\text { DR } \\
\mathrm{N}=373\end{array}$ & $\begin{array}{c}\mathrm{p} \text {-values } \\
\text { group diff/lin. trend }\end{array}$ & $\begin{array}{c}\text { Effect Size } \\
w\end{array}$ \\
\hline Any Axis I DX [\% (n)] & $51.4(179)$ & $70.0(252)^{*}$ & $74.9(262)^{*}$ & $<.001 /<.001$ & .211 \\
\hline Any Axis II DX [\% (n)] & $53.8(184)$ & $59.2(209)$ & $58.7(202)$ & $.284 / .194$ & \\
\hline Dual Axis DX [\%(n)] & $34.0(115)$ & $45.0(158)^{*}$ & $48.4(165)^{*}$ & $<.001 /<.001$ & .123 \\
\hline $\operatorname{MDD}[\%(n)]$ & $43.4(151)$ & $61.1(220)^{*}$ & $62.6(219)^{*}$ & $<.001 /<.001$ & .175 \\
\hline Anxiety Disorder [\% (n)] & $12.4(43)$ & $16.9(61)$ & $18.9(66)$ & $.056 / .019$ & \\
\hline PTSD (\% (n)] & $3.2(11)$ & $1.9(7)$ & $4.9(17)$ & $.093 / .209$ & \\
\hline $\begin{array}{l}\text { Substance Use Disorders [\% (n)] } \\
\text { Alcohol Abuse } \\
\text { Alcohol Dependence } \\
\text { Drug Abuse (excl. Opioid) } \\
\text { Drug Dependence (excl. Opioid) } \\
\text { Opioid Abuse } \\
\text { Opioid Dependence }\end{array}$ & $\begin{array}{c}0.6(2) \\
1.7(6) \\
0 \\
0.3(1) \\
0 \\
13.5(47)\end{array}$ & $\begin{array}{c}0.8(3) \\
1.9(7) \\
0.3(1) \\
2.2(8) \\
0 \\
18.9(68)\end{array}$ & $\begin{array}{c}3.1(11)^{*} \\
2.0(7) \\
0 \\
0.3(1) \\
0.3(1) \\
29.1(102)^{*}\end{array}$ & $\begin{array}{c}.009 / .005 \\
.961 / .789 \\
.379 / .998 \\
.010 / .994 \\
.363 / .219 \\
<.001 /<.001\end{array}$ & $\begin{array}{l}.095 \\
.160\end{array}$ \\
\hline
\end{tabular}


Table A.12 - Continued

\begin{tabular}{|l|c|c|c|c|c|}
\hline Paranoid PD [\% (n)] & $17.8(61)$ & $17.0(60)$ & $19.8(68)$ & $.625 / .512$ & \\
\hline Borderline PD [\% (n)] & $18.1(62)$ & $21.8(77)$ & $21.2(73)$ & $.435 / .316$ & \\
\hline Histrionic PD [\% (n)] & $7.9(27)$ & $10.5(37)$ & $9.6(33)$ & $.493 / .446$ & \\
\hline Obsessive-Compulsive PD [\% (n)] & $14.9(51)$ & $15.0(53)$ & $20.9(72)$ & $.054 / .036$ & \\
\hline
\end{tabular}

* Significantly different compared to ER group, at $p<.05$ after adjusting for inflation of Type I error 
Table A.13. Effect Sizes for Post-Hoc Comparisons of Significant Psychiatric Diagnoses $(\mathrm{N}=1119)$

\begin{tabular}{|c|c|c|}
\hline \multirow{2}{*}{ Variables } & \multicolumn{2}{|c|}{ Odds Ratio $(95 \%$ CI) } \\
\cline { 2 - 3 } & ID vs. ER & DR vs. ER \\
\hline Any Axis I DX & $2.2(1.6,3.0)$ & $2.8(2.0,3.9)$ \\
\hline Dual Axis DX & $1.6(1.2,2.2)$ & $1.8(1.3,2.5)$ \\
\hline MDD & $2.1(1.5,2.8)$ & $2.2(1.6,3.0)$ \\
\hline Alcohol Abuse & NS & $5.6(1.2,25.5)$ \\
\hline Opioid Dependence & NS & $2.6(1.8,3.9)$ \\
\hline
\end{tabular}


Table A.14. Pre-Rehabilitation Psychosocial Measures $(\mathrm{N}=1119)$

\begin{tabular}{|c|c|c|c|c|c|}
\hline Variables & $\begin{array}{c}\text { ER } \\
N=373\end{array}$ & $\begin{array}{c}\text { ID } \\
\mathrm{N}=373\end{array}$ & $\begin{array}{c}\text { DR } \\
N=373\end{array}$ & $\begin{array}{c}\text { p-values } \\
\text { group diff/lin. trend }\end{array}$ & $\begin{array}{l}\text { Effect Size } \\
\eta^{2} \text { or } w\end{array}$ \\
\hline $\begin{array}{l}\text { HAM-D (SD) } \\
\quad \geq 14[\%(\mathrm{n})]\end{array}$ & $\begin{array}{l}15.6(5.7) \\
67.6(250)\end{array}$ & $\begin{array}{l}17.5(5.7)^{*} \\
76.4(285)^{*}\end{array}$ & $\begin{array}{l}18.0(5.4)^{*} \\
81.5(303)^{*}\end{array}$ & $\begin{array}{l}<.001 /<.001 \\
<.001 /<.001\end{array}$ & $\begin{array}{l}.033 \\
.133\end{array}$ \\
\hline $\begin{array}{l}\text { Pain Intensity VAS (SD) } \\
\quad \geq 8[\%(\mathrm{n})]\end{array}$ & $\begin{array}{c}6.6(1.8) \\
38.8(143)\end{array}$ & $\begin{array}{c}6.5(1.8) \\
34.1(126)\end{array}$ & $\begin{array}{c}6.8(1.9) \\
43.9(161)\end{array}$ & $\begin{array}{l}.050 / .103 \\
.024 / .156\end{array}$ & .082 \\
\hline $\begin{array}{l}\text { Million VAS (SD) } \\
\geq 101[\%(\mathrm{n})]\end{array}$ & $\begin{array}{l}90.7(26.9) \\
39.7(145)\end{array}$ & $\begin{array}{l}93.5(23.9) \\
41.7(153)\end{array}$ & $\begin{array}{l}96.8(23.8)^{*} \\
49.0(177)^{*}\end{array}$ & $\begin{array}{l}.005 / .001 \\
.029 / .012\end{array}$ & $\begin{array}{l}.010 \\
.081\end{array}$ \\
\hline $\begin{array}{l}\text { SF-36 (SD) } \\
\text { Mental Component } \\
\text { Physical Component } \\
\text { Physical Functioning } \\
\text { Role Physical } \\
\text { Bodily Pain } \\
\text { General Health } \\
\text { Vitality } \\
\text { Social Functioning } \\
\text { Role Emotional } \\
\text { Mental Health }\end{array}$ & $\begin{array}{c}39.7(9.5) \\
29.9(6.1) \\
35.6(19.4) \\
10.4(17.8) \\
27.4(11.7) \\
52.8(18.2) \\
35.9(17.5) \\
42.7(21.9) \\
32.4(30.1) \\
54.3(17.7)\end{array}$ & $\begin{array}{c}38.1(10.0) \\
29.9(5.7) \\
34.5(17.7) \\
8.3(17.8) \\
27.2(11.5) \\
50.5(16.8) \\
35.7(17.5) \\
40.1(21.5) \\
27.9(30.7) \\
51.6(19.3)\end{array}$ & $\begin{array}{c}38.5(9.4) \\
30.2(5.4) \\
37.0(17.1) \\
9.2(16.5) \\
27.3(11.5) \\
50.7(16.8) \\
33.5(16.1) \\
41.9(20.0) \\
30.4(29.8) \\
52.8(17.5)\end{array}$ & $\begin{array}{l}.122 / .137 \\
.813 / .546 \\
.231 / . .344 \\
.362 / .440 \\
.966 / .905 \\
.216 / .158 \\
.168 / .094 \\
.322 / .662 \\
.203 / .431 \\
.195 / .283\end{array}$ & \\
\hline
\end{tabular}


Table A.14 - Continued

\begin{tabular}{|l|l|l|l|l|l|}
\hline $\begin{array}{l}\text { Pre-Treatment Quality of } \\
\text { Life Scores (SD) }\end{array}$ & $0.58(0.03)$ & $0.58(0.03)$ & $0.58(0.02)$ & $.295 / .324$ & \\
\hline
\end{tabular}

* Significantly different compared to ER group, at $p<.05$ after adjusting for inflation of Type I error 
Table A.15. Effect Sizes for Post-Hoc Comparisons of Significant Pre-rehabilitation Psychosocial Measures $(\mathrm{N}=1119)$

\begin{tabular}{|l|c|c|}
\hline \multirow{2}{*}{ Variables } & \multicolumn{2}{|c|}{ Cohen's $d$ or Odds Ratio $(95 \%$ CI) } \\
\cline { 2 - 3 } & ID vs. ER & DR vs. ER \\
\hline $\begin{array}{c}\text { PRE HAM-D } \\
\geq 14[\%(n)]\end{array}$ & 0.3 & 0.4 \\
& $1.6(1.1,2.1)$ & $2.1(1.5,3.0)$ \\
\hline $\begin{array}{l}\text { PRE Million VAS } \\
\geq 101[\%(n)]\end{array}$ & NS & 0.2 \\
& NS & $1.5(1.1,2.0)$ \\
\hline
\end{tabular}


Table A.16. Post-Rehabilitation Psychosocial Measures $(\mathrm{N}=1119)$

\begin{tabular}{|c|c|c|c|c|c|}
\hline Variables & $\begin{array}{c}\text { ER } \\
N=373\end{array}$ & $\begin{array}{c}\text { ID } \\
N=373\end{array}$ & $\begin{array}{c}\text { DR } \\
N=373\end{array}$ & $\begin{array}{c}\text { p-values } \\
\text { group diff/lin. trend }\end{array}$ & $\begin{array}{l}\text { Effect Size } \\
\quad \eta^{2} \text { or } w\end{array}$ \\
\hline $\begin{array}{l}\text { HAM-D (SD) } \\
\quad \geq 14[\%(\mathrm{n})]\end{array}$ & $\begin{array}{l}7.9(4.4) \\
12.6(38)\end{array}$ & $\begin{array}{l}8.7(4.2) \\
15.0(46)\end{array}$ & $\begin{array}{c}9.4(4.5) \\
20.9(60)^{*}\end{array}$ & $\begin{array}{l}.072 / .031 \\
.019 / .006\end{array}$ & .094 \\
\hline $\begin{array}{l}\text { Pain Intensity VAS (SD) } \\
\quad \geq 8[\%(\mathrm{n})]\end{array}$ & $\begin{array}{l}4.2(2.2) \\
8.0(27)\end{array}$ & $\begin{array}{l}4.3(2.2) \\
10.1(34)\end{array}$ & $\begin{array}{l}4.4(2.2) \\
9.5(32)\end{array}$ & $\begin{array}{l}.656 / .479 \\
.628 / .497\end{array}$ & \\
\hline $\begin{array}{l}\text { Million VAS (SD) } \\
\quad \geq 101[\%(\mathrm{n})]\end{array}$ & $\begin{array}{c}56.6(29.6) \\
8.3(28)\end{array}$ & $\begin{array}{l}59.2(29.3) \\
8.4(28)\end{array}$ & $\begin{array}{c}61.9(30.5) \\
13.3(44)\end{array}$ & $\begin{array}{l}.612 / .326 \\
.050 / .033\end{array}$ & \\
\hline $\begin{array}{l}\text { SF-36 (SD) } \\
\text { Mental Component } \\
\text { Physical Component } \\
\text { Physical Functioning } \\
\text { Role Physical } \\
\text { Bodily Pain } \\
\text { General Health } \\
\text { Vitality } \\
\text { Social Functioning } \\
\text { Role Emotional } \\
\text { Mental Health }\end{array}$ & $\begin{array}{c}47.0(8.9) \\
36.8(7.8) \\
57.9(20.6) \\
29.9(33.2) \\
42.2(15.9) \\
63.2(17.7) \\
51.9(17.5) \\
63.0(21.6) \\
54.2(33.7) \\
68.1(16.5)\end{array}$ & $\begin{array}{c}46.7(9.9) \\
37.3(8.3) \\
58.0(20.6) \\
34.5(33.0)) \\
45.0(16.2) \\
62.1(17.2) \\
52.7(18.1) \\
60.9(22.1) \\
54.7(34.6) \\
68.2(17.6)\end{array}$ & $\begin{array}{c}47.3(10.2) \\
36.6(8.3) \\
57.6(20.3) \\
31.0(32.4) \\
44.6(16.4) \\
61.7(17.4) \\
51.9(17.6) \\
62.3(22.5) \\
55.1(36.3) \\
69.2(17.3)\end{array}$ & $\begin{array}{l}.833 / .742 \\
.590 / .876 \\
.862 / .824 \\
.360 / .754 \\
.213 / .144 \\
.801 / .507 \\
.787 / .939 \\
.725 / .798 \\
.940 / .748 \\
.789 / .496\end{array}$ & \\
\hline
\end{tabular}


Table A.16 - Continued

\begin{tabular}{|l|l|l|l|l|l|}
\hline $\begin{array}{l}\text { Post-Treatment Quality } \\
\text { of Life Scores (SD) }\end{array}$ & $0.63(0.05)$ & $0.63(0.05)$ & $0.63(0.06)$ & $.748 / .807$ & \\
\hline
\end{tabular}

* Significantly different compared to ER group, at $p<.05$ after adjusting for inflation of Type I error 
Table A.17. One-Year Socioeconomic Outcomes $(\mathrm{N}=1119)$

\begin{tabular}{|c|c|c|c|c|c|}
\hline Variables & $\begin{array}{c}\text { ER } \\
\mathrm{N}=337\end{array}$ & $\begin{array}{c}\text { ID } \\
\mathrm{N}=337\end{array}$ & $\begin{array}{c}\text { DR } \\
\mathrm{N}=337\end{array}$ & $\begin{array}{c}\mathrm{p} \text {-values } \\
\text { group diff/lin. trend }\end{array}$ & $\begin{array}{l}\text { Effect Size } \\
\quad \eta^{2} \text { or } w\end{array}$ \\
\hline $\operatorname{RTW}[\%(\mathrm{n})]$ & $89.4(287)$ & $88.3(288)$ & $84.8(284)$ & $.170 / .072$ & \\
\hline Work Retention [\% (n)] & $82.5(259)$ & $82.3(265)$ & $76.4(252)$ & $.082 / .049$ & \\
\hline $\begin{array}{l}\text { Seeking Treatment from } \\
\text { New Healthcare Provider } \\
{[\%(n)]}\end{array}$ & $18.7(61)$ & $18.4(61)$ & $17.9(60)$ & $.960 / .776$ & \\
\hline $\begin{array}{l}\text { Visits to New Healthcare } \\
\text { Provider (SD) }\end{array}$ & $2.0(6.2)$ & $1.7(5.2)$ & $1.9(6.1)$ & $.776 / .791$ & \\
\hline $\begin{array}{l}\text { New Surgery for Original } \\
\text { Injury }[\%(\mathrm{n})]\end{array}$ & $1.5(5)$ & $2.4(8)$ & $2.7(9)$ & $.570 / .310$ & \\
\hline $\begin{array}{l}\text { Recurrent Injury with Lost } \\
\text { Time }[\%(n)]\end{array}$ & $2.3(7)$ & $0.9(3)$ & $0.9(3)$ & $.244 / .147$ & \\
\hline Case Settled [\% (n)] & $96.5(331)$ & $98.8(337)$ & $96.5(335)$ & $.101 / .980$ & \\
\hline
\end{tabular}


Table A.17 - Continued

\begin{tabular}{|l|c|c|c|c|c|}
\hline Disability Income [\% (n)] & & & & & \\
TTD Benefits & $3.0(9)$ & $1.6(5)$ & $3.2(10)$ & $.386 / .845$ & $.003 / .001$ \\
Impairment Benefits & $3.3(10)$ & $8.9(28)^{*}$ & $10.2(32)^{*}$ & .0013 \\
STD/LTD & $1.6(5)$ & $1.9(6)$ & $2.9(9)$ & $.535 / .290$ & \\
SSDI/SSI & $0.3(1)$ & $4.4(14)^{*}$ & $9.3(29)^{*}$ & $<.001 /<.001$ & .172 \\
SIBS & 0 & $0.3(1)$ & $6.7(21)$ & $<.001 /<.001$ & .204 \\
\hline
\end{tabular}

* Significantly different compared to ER group, at $p<.05$ after adjusting for inflation of Type I error 
Table A.18. Effect Sizes for Post-Hoc Comparisons of Significant One-Year Outcomes (N = 1119)

\begin{tabular}{|l|c|c|}
\hline \multirow{2}{*}{ Variables } & \multicolumn{2}{|c|}{ Cohen's $d$ or Odds Ratio (95\% CI) } \\
\cline { 2 - 3 } & ID vs. ER & DR vs. ER \\
\hline Impairment Benefits & $2.9(1.4,6.0)$ & $3.3(1.6,6.9)$ \\
\hline SSDI & $14.0(1.8,107.5)$ & $30.9(4.2,228.6)$ \\
\hline SIBS & NS & $21.8(2.9,163.0)$ \\
\hline
\end{tabular}


Table A.19. Costs Associated with the PRIDE Functional Restoration Program $(\mathrm{N}=1119)$

\begin{tabular}{|c|c|c|c|c|c|}
\hline Variables & $\begin{array}{c}\text { ER } \\
N=373\end{array}$ & $\begin{array}{c}\text { ID } \\
\mathrm{N}=373\end{array}$ & $\begin{array}{c}\text { DR } \\
\mathrm{N}=373\end{array}$ & $\begin{array}{c}\text { p-values } \\
\text { group diff/lin. trend }\end{array}$ & $\begin{array}{c}\text { Effect Size } \\
\eta^{2}\end{array}$ \\
\hline $\begin{array}{l}\text { Total PRIDE Cost } \\
\text { Median (IQR) } \\
\text { \$(SD) } \\
\text { Log \$ (SD) }\end{array}$ & $\begin{array}{c}\$ 29,065(\$ 4,009) \\
\$ 28,994(\$ 5,072) \\
10.2(0.2)\end{array}$ & $\begin{array}{c}\$ 29,967(\$ 3,617) \\
\$ 30,342(\$ 4,608) \\
10.3(0.1)^{*}\end{array}$ & $\begin{array}{c}\$ 30,179(\$ 4,475) \\
\$ 30,195(\$ 6,294) \\
10.3(0.2)^{*}\end{array}$ & $<.001 / .007$ & .014 \\
\hline $\begin{array}{l}\text { Functional Restoration } \\
\text { Median (IQR) } \\
\text { Program Costs, \$ (SD) } \\
\text { Log } \$(\mathrm{SD}) \\
\text { Program Hours (SD) }\end{array}$ & $\begin{array}{c}\$ 24,939(\$ 4,330) \\
\$ 24,499(\$ 4,330) \\
10.1(0.2) \\
160(28.4)\end{array}$ & $\begin{array}{c}\$ 25,910(\$ 2,808) \\
\$ 25,997(\$ 3,916) \\
10.2(0.1)^{*} \\
176(26.8)^{*}\end{array}$ & $\begin{array}{c}\$ 26,233(\$ 4,018) \\
\$ 26,188(\$ 5,683) \\
10.2(0.2)^{*} \\
177(34.6)^{*}\end{array}$ & $\begin{array}{l}<.001 /<.001 \\
<.001 /<.001\end{array}$ & $\begin{array}{l}.025 \\
.062\end{array}$ \\
\hline $\begin{array}{l}\text { Consultation \& Office Visits } \\
\text { Median (IQR) } \\
\text { \$(SD) } \\
\text { Log \$ (SD) }\end{array}$ & $\begin{array}{l}\$ 3,695(\$ 1,817) \\
\$ 4,145(\$ 2,230) \\
8.2(0.4)\end{array}$ & $\begin{array}{c}\$ 3,597(\$ 1,776) \\
\$ 3,914(\$ 1,547) \\
8.2(0.4)\end{array}$ & $\begin{array}{c}\$ 3,285(\$ 1,686) \\
\$ 3,557(\$ 1,507) \\
8.1(0.4)^{*}\end{array}$ & $<.001 /<.001$ & .023 \\
\hline $\begin{array}{l}\text { Therapeutic Injections } \\
\mathrm{N} \\
\text { Median (IQR) } \\
\text { \$ (SD) }\end{array}$ & $\begin{array}{c}15 \\
\$ 508(\$ 442) \\
\$ 683(\$ 440)\end{array}$ & $\begin{array}{c}18 \\
\$ 538(\$ 178) \\
\$ 616(\$ 306)\end{array}$ & $\begin{array}{c}17 \\
\$ 528(\$ 128) \\
\$ 581(\$ 309)\end{array}$ & $.714 / .420$ & \\
\hline
\end{tabular}


Table A.19 - Continued

\begin{tabular}{|l|c|c|c|c|}
\hline Admission Medications & & & & \\
$\mathrm{N}$ & 291 & 313 & 303 & \\
Median (IQR) & $\$ 195(\$ 191)$ & $\$ 224(\$ 187)$ & $\$ 243(\$ 209)$ & \\
\$(SD) & $\$ 215(\$ 160)$ & $\$ 257(\$ 160)^{*}$ & $\$ 267(\$ 162)^{*}$ & $<.001 /<.001$ \\
\hline \\
\hline Discharge Medications & 270 & 289 & 284 & \\
$\mathrm{~N}$ & $\$ 198(\$ 199)$ & $\$ 227(\$ 187)$ & $\$ 238(\$ 212)$ & \\
Median (IQR) & $\$ 226(\$ 198)$ & $\$ 253(\$ 155)$ & $\$ 272(\$ 176)^{*}$ & .021 \\
$\$(\mathrm{SD})$ & & & & $.009 / .002$ \\
\hline
\end{tabular}

* Significantly different compared to ER group, at $p<.05$ after adjusting for inflation of Type I error 
Table A.20. Effect Sizes for Post-Hoc Comparisons of Significant Costs Associated with the PRIDE Functional Restoration Program $(\mathrm{N}=1119)$

\begin{tabular}{|l|c|c|}
\hline \multirow{2}{*}{ Variables } & \multicolumn{2}{|c|}{ Cohen's $d$} \\
\cline { 2 - 3 } & ID vs. ER & DR vs. ER \\
\hline Total PRIDE Cost & 0.3 & 0.2 \\
\hline $\begin{array}{l}\text { Functional Restoration } \\
\text { Program Costs } \\
\text { Program Hours }\end{array}$ & 0.3 & 0.3 \\
& 0.5 & 0.6 \\
\hline Consultation \& Office Visits & NS & -0.4 \\
\hline Admission Medications & 0.3 & 0.3 \\
\hline Discharge Medications & NS & 0.3 \\
\hline
\end{tabular}


Table A.21. Costs Incurred at One-Year Post-Rehabilitation $(\mathrm{N}=1119)$

\begin{tabular}{|c|c|c|c|c|}
\hline Variables & $\begin{array}{c}\text { ER } \\
\mathrm{N}=373\end{array}$ & $\begin{array}{c}\text { ID } \\
N=373\end{array}$ & $\begin{array}{c}\text { DR } \\
\mathrm{N}=373\end{array}$ & $\begin{array}{c}\text { p-values } \\
\text { group diff/lin. trend }\end{array}$ \\
\hline $\begin{array}{l}\text { Cost of Visits to New } \\
\text { Healthcare Providers } \\
\text { N } \\
\text { Median (IQR) } \\
\text { \$ (SD) } \\
\text { Log \$ (SD) }\end{array}$ & $\begin{array}{c}61 \\
\$ 225(\$ 1126) \\
\$ 822(\$ 793) \\
6.3(0.9)\end{array}$ & $\begin{array}{c}61 \\
\$ 600(\$ 750) \\
\$ 706(\$ 654) \\
6.2(0.8)\end{array}$ & $\begin{array}{c}60 \\
\$ 413(\$ 750) \\
\$ 810(\$ 225) \\
6.3(0.9)\end{array}$ & $.917 / .964$ \\
\hline $\begin{array}{l}\text { Cost of New Surgeries for } \\
\text { Original Injury } \\
\text { N } \\
\text { Median (IQR) } \\
\text { \$ (SD) } \\
\text { Log } \$(\mathrm{SD})\end{array}$ & $\begin{array}{c}4 \\
\$ 12,149(\$ 11,553) \\
\$ 11,795(\$ 6,491) \\
9.2(0.6)\end{array}$ & $\begin{array}{c}7 \\
\$ 16,060(\$ 12,392) \\
\$ 12,933(\$ 7,873) \\
9.2(1.0)\end{array}$ & $\begin{array}{c}9 \\
\$ 6,904(\$ 5,728) \\
\$ 9,366(\$ 3,537) \\
9.1(0.3)\end{array}$ & $.926 / .712$ \\
\hline
\end{tabular}


Table A.22. Costs Associated with the Analytic Timeframe of the Cost-Effectiveness Analysis $(\mathrm{N}=1119)$

\begin{tabular}{|c|c|c|c|c|c|}
\hline Variables & $\begin{array}{c}\text { ER } \\
N=373\end{array}$ & $\begin{array}{c}\text { ID } \\
N=373\end{array}$ & $\begin{array}{c}\text { DR } \\
N=373\end{array}$ & $\begin{array}{c}\mathrm{p} \text {-values } \\
\text { group diff/lin. trend }\end{array}$ & $\begin{array}{c}\text { Effect Size } \\
\eta^{2}\end{array}$ \\
\hline $\begin{array}{l}\text { Cost within Analytic Timeframe } \\
\text { Median \$ (IQR) } \\
\text { \$ (SD) } \\
\text { Log \$ (SD) }\end{array}$ & $\begin{array}{c}\$ 29,260(\$ 4,210) \\
\$ 29,255(\$ 5,301) \\
10.2(0.2)\end{array}$ & $\begin{array}{c}\$ 30,156(\$ 3,931) \\
\$ 30,700(\$ 5,143) \\
10.3(0.2)^{*}\end{array}$ & $\begin{array}{c}\$ 30,347(\$ 4,761) \\
\$ 30,553(\$ 6,818) \\
10.3(0.2)^{*}\end{array}$ & $<.001 / .007$ & .014 \\
\hline
\end{tabular}

* Significantly different compared to ER group, at $p<.05$ after adjusting for inflation of Type I error

Table A.23. Effect Sizes for Post-Hoc Comparisons of Costs within the Analytic Timeframe $(\mathrm{N}=1119)$

\begin{tabular}{|l|c|c|}
\hline \multirow{2}{*}{ Variables } & \multicolumn{2}{|c|}{ Cohen's $d$} \\
\cline { 2 - 3 } & ID vs. ER & DR vs. ER \\
\hline $\begin{array}{l}\text { Cost within Analytic } \\
\text { Timeframe }\end{array}$ & 0.3 & 0.2 \\
\hline
\end{tabular}


Table A.24. Average Cost-Effectiveness Ratio for the Analytical Timeframe based on RTW

\begin{tabular}{lcccc}
\hline Group & Total Cost & \% RTW & Average Ratio & $(\mathbf{9 5 \%}$ CI) \\
\hline ER & $\$ 29,255$ & 0.89 & $\$ 32,711$ & $(\$ 31,390 ; \$ 34,206)$ \\
ID & $\$ 30,700$ & 0.88 & $\$ 34,765$ & $(\$ 33,255 ; \$ 36,345)$ \\
DR & $\$ 30,553$ & 0.85 & $\$ 36,051$ & $(\$ 34,264 ; \$ 38,080)$
\end{tabular}

Table A.25. Incremental Cost-Effectiveness Ratio for the Analytical Timeframe based on RTW

\begin{tabular}{llccccc}
\hline & Group & Cost per Group & \% RTW & $\Delta$ Cost & $\Delta$ Effectiveness & ICER \\
\hline I. & DR & $\$ 30,553$ & 0.85 & & & \\
& ID & $\$ 30,700$ & 0.88 & $\$ 147$ & 0.04 & $\$ 4,130$ \\
& ER & $\$ 29,255$ & 0.89 & $-\$ 1,445$ & 0.01 & $-\$ 128,353$ \\
& & & & & & \\
II. & DR & $\$ 30,553$ & 0.85 & & 0.05 & $-\$ 27,703$ \\
& ER & $\$ 29,255$ & 0.89 & $-\$ 1,298$ & & \\
\hline
\end{tabular}

Table A.26. Joint Distribution of Incremental Costs and Incremental Effectives based on RTW

\begin{tabular}{lccc}
\hline Quadrants & ER vs. ID & ER vs. DR & ID vs. DR \\
\hline & & & \\
1. More Costly-More Effective & .00 & .00 & .58 \\
2. More Costly-Less Effective & .00 & .00 & .05 \\
3. Less Costly-Less Effective & .32 & .04 & .03 \\
4. Less Costly-More Effective & .68 & .96 & .34 \\
\hline
\end{tabular}


Table A.27. Average Cost-Effectiveness Ratio for the Analytical Timeframe based on Work Retention

\begin{tabular}{lcccc}
\hline Group & Total Cost & \% Retained Work & Average Ratio & (95\% CI) \\
\hline ER & $\$ 29,255$ & 0.83 & $\$ 35,442$ & $(\$ 33,563 ; \$ 37,599)$ \\
ID & $\$ 30,700$ & 0.82 & $\$ 37,300$ & $(\$ 35,311 ; \$ 39,392)$ \\
DR & $\$ 30,553$ & 0.76 & $\$ 40,201$ & $(\$ 37,503 ; \$ 43,131)$
\end{tabular}

Table A.28. Incremental Cost-Effectiveness Ratio for the Analytical Timeframe based on Work Retention

\begin{tabular}{llccccc}
\hline & Group & Total Cost & \% Retained Work & $\Delta$ Cost & $\Delta$ Effectiveness & ICER \\
\hline I. & DR & $\$ 30,553$ & 0.76 & & & \\
& ID & $\$ 30,700$ & 0.82 & $\$ 147$ & 0.06 & $\$ 2,480$ \\
& ER & $\$ 29,255$ & 0.83 & $-\$ 1,445$ & $<0.01$ & $-\$ 606,379$ \\
& & & & & \\
II. & DR & $\$ 30,553$ & 0.76 & $-\$ 1,298$ & 0.07 & $-\$ 18,543$ \\
& ER & $\$ 29,255$ & 0.83 & & & \\
\hline
\end{tabular}

Table A.29. Joint Distribution of Incremental Costs and Incremental Effectives based on Work Retention

\begin{tabular}{lccc}
\hline Quadrants & ER vs. ID & ER vs. DR & ID vs. DR \\
\hline & & & \\
1. More Costly-More Effective & .00 & .00 & .62 \\
2. More Costly-Less Effective & .00 & .00 & .01 \\
3. Less Costly-Less Effective & .46 & .02 & .01 \\
4. Less Costly-More Effective & .54 & .98 & .36 \\
\hline
\end{tabular}


Table A.30. Average Cost-Utility Ratio for the Analytical Timeframe based on Post-Treatment Changes in Quality-of-Life

\begin{tabular}{lcccc}
\hline Group & Total Cost & $\Delta$ QoL (PRE - POST) & Average Ratio & $(\mathbf{9 5 \%}$ CI) \\
\hline ER & $\$ 29,255$ & 0.05 & $\$ 585,100$ & $(\$ 546,752 ; \$ 764,108)$ \\
ID & $\$ 30,700$ & 0.05 & $\$ 614,000$ & $(\$ 532,920 ; \$ 706,145)$ \\
DR & $\$ 30,553$ & 0.05 & $\$ 611,060$ & $(\$ 574,519 ; \$ 775,667)$
\end{tabular}

Table A.31. Incremental Cost-Utility Ratio for the Analytical Timeframe based on Post-Treatment Changes in Quality-of-Life

\begin{tabular}{llcccccc}
\hline & Group & Total Cost & $\Delta$ QoL (PRE - POST) & $\Delta$ Cost & $\Delta$ Effectiveness & ICER & (95\% CI) \\
\hline I. & DR & $\$ 30,553$ & 0.05 & & & & N/A \\
& ID & $\$ 30,700$ & 0.05 & $\$ 147$ & 0.00 & N/A & N/A \\
& ER & $\$ 29,255$ & 0.05 & $-\$ 1,445$ & 0.00 & & N/A \\
II. & DR & $\$ 30,553$ & 0.05 & & & & N/A \\
& ER & $\$ 29,255$ & 0.05 & $-\$ 1,298$ & 0.00 & & \\
\end{tabular}


Table A.32. Correlation Matrix of Predictors Utilized in the Sequential Regression Analysis

\begin{tabular}{|c|c|c|c|c|c|c|c|c|c|c|c|}
\hline & $\begin{array}{c}\text { ID } \\
\text { Group }\end{array}$ & $\begin{array}{c}\text { DR } \\
\text { Group }\end{array}$ & $\begin{array}{c}\text { Pre } \\
\text { HAM-D }\end{array}$ & $\begin{array}{c}\text { Pre } \\
\text { MVAS }\end{array}$ & MDD & $\begin{array}{c}\text { Alc } \\
\text { Abuse }\end{array}$ & $\begin{array}{l}\text { Opi } \\
\text { Dep }\end{array}$ & $\begin{array}{l}\text { Drug } \\
\text { Dep }\end{array}$ & $\begin{array}{c}\text { Job } \\
\text { Avail }\end{array}$ & Attorney & $\begin{array}{c}\text { Case } \\
\text { Settled }\end{array}$ \\
\hline ID Group & 1 & & & & & & & & & & \\
\hline DR Group & -.50 & 1 & & & & & & & & & \\
\hline Pre HAM-D & .05 & .12 & 1 & & & & & & & & \\
\hline Pre MVAS & -.01 & .09 & .31 & 1 & & & & & & & \\
\hline MDD & .08 & .10 & .43 & .21 & 1 & & & & & & \\
\hline Alc Abuse & -.04 & .09 & .02 & .00 & .03 & 1 & & & & & \\
\hline Opi Dep & -.03 & .15 & .17 & .22 & .12 & .07 & 1 & & & & \\
\hline Drug Dep & .10 & -.05 & .07 & .01 & .05 & -.01 & -.05 & 1 & & & \\
\hline Job Avail & -.03 & -.21 & -.15 & -.14 & -.09 & -.01 & -.07 & -.02 & 1 & & \\
\hline Attorney & .07 & .08 & .08 & .09 & .11 & .03 & .03 & .05 & -.16 & 1 & \\
\hline Case Settled & -.11 & .43 & .11 & .13 & .08 & -.01 & .11 & .03 & -.22 & .07 & 1 \\
\hline Surgery & .05 & .33 & .06 & -.05 & .06 & .05 & .10 & -.01 & -.11 & .00 & .12 \\
\hline
\end{tabular}


Table A.33. Predictors of Costs within the Analytic Timeframe

\begin{tabular}{llccccc}
\hline Block & Variables & B & Std. & & & \\
\hline 1 & Constant & Error & $\mathrm{t}$ & $\mathrm{p}$ & $\beta$ \\
\hline Adj. $R^{2}=$ & & 29282.3 & 321.3 & 91.1 & $<.001$ & \\
& ID Group & & & & & \\
& DR Group & 1481.2 & 449.4 & 3.3 & .001 & 0.12 \\
& & 1423.1 & 458.6 & 3.1 & .002 & 0.113 \\
\hline Adj. $R^{2}=$ & & & & & \\
.052 & Constant & 26391.7 & 899.5 & 29.3 & $<.001$ & \\
& ID Group & & & & & \\
& DR Group & 1020.2 & 473.6 & 2.2 & .031 & .08 \\
& Pre HAM-D & 1291 & 564.6 & 2.3 & .022 & .10 \\
& Pre MVAS & 46 & 36.6 & 1.3 & .209 & .04 \\
& Major Depressive Disorder & 1364.2 & 409.8 & 3.3 & .001 & .12 \\
& Alcohol Abuse Disorder & -1688.4 & 1452.6 & -1.2 & .245 & -.04 \\
& Opioid Dependence & & & & & \\
& Disordeer & -438.4 & 466.4 & -0.9 & .347 & -.03 \\
& Drug Dependence Disorder & -419 & 1929.0 & -0.2 & .828 & -.01 \\
& Job Availability & 41.8 & 383.8 & 0.1 & .913 & .00 \\
& Attorney Retained & 343.9 & 463.9 & 0.7 & .459 & .02 \\
& Case Settled at Admission & -1777.2 & 466.4 & -3.8 & .000 & -.13 \\
& Prior Surgery & 1240.3 & 403.9 & 3.1 & .002 & .11 \\
& & & & & \\
\hline
\end{tabular}


Table A.33 - Continued

\begin{tabular}{llccccc}
\hline $\begin{array}{l}\text { Final Model } \\
\text { Adj. } R^{2}=\end{array}$ & Constant & 26834.0 & 745.5 & 36.0 & .000 & \\
.056 & Case Settled at Admission & -1686.3 & 455.0 & -3.7 & .000 & -.13 \\
& Major Depressive Disorder & 1496.6 & 375.8 & 4.0 & .000 & .13 \\
& Prior Surgery & 1203.7 & 399.8 & 3.0 & .003 & .10 \\
& DR Group & 1117.0 & 545.6 & 2.0 & .041 & .09 \\
& Pre MVAS & 19.6 & 7.5 & 2.6 & .009 & .08 \\
& ID Group & 923.0 & 463.0 & 2.0 & .046 & .07 \\
& & & & & \\
\hline
\end{tabular}


Table A.34. Characteristics of the OBWC Cohort compared to the PRIDE Cohort

\begin{tabular}{|c|c|c|c|c|}
\hline Variables & $\begin{array}{c}\text { OBWC } \\
\text { Unmatched } \\
\mathrm{N}=4,726\end{array}$ & $\begin{array}{c}\text { OBWC } \\
\text { Initial Match } \\
\mathrm{N}=1,119\end{array}$ & $\begin{array}{c}\text { OBWC } \\
\text { Final Match } \\
\mathrm{N}=925\end{array}$ & $\begin{array}{c}\text { PRIDE } \\
\text { Cohort } \\
\mathrm{N}=1,119\end{array}$ \\
\hline Gender $[\%$ Male $(n)]$ & $58.0(2741)$ & $64.0(714)$ & $65.5(605)$ & $53.1(594)^{*}$ \\
\hline $\begin{array}{l}\text { Age Groups }[\%(\mathrm{n})] \\
\quad \leq 30 \\
31-40 \\
41-50 \\
51-60 \\
>60\end{array}$ & $\begin{array}{c}15.8(745) \\
28.9(1368) \\
32.4(1530) \\
18.1(854) \\
4.7(220)\end{array}$ & $\begin{array}{c}14.7(164) \\
31.3(350) \\
33.2(371) \\
16.9(189) \\
3.8(43)\end{array}$ & $\begin{array}{c}15.2(141) \\
32.3(299) \\
32.5(301) \\
15.8(146) \\
4.1(38)\end{array}$ & $\begin{array}{c}1.9(21)^{*} \\
25.2(282) \\
43.4(486) \\
21.6(483) \\
3.2(36)\end{array}$ \\
\hline $\begin{array}{l}\text { Injured Musculoskeletal Regions [\% (n)] } \\
\text { Cervical only } \\
\text { Lumbar/Thoracic only } \\
\text { Multiple Musculoskeletal } \\
\text { Upper/Lower Extremities only } \\
\text { Multiple Musculoskeletal (at least } 1 \text { spinal) }\end{array}$ & $\begin{array}{c}1.3(60) \\
12.1(574) \\
2.0(96) \\
61.0(2885) \\
22.7(1074)\end{array}$ & $\begin{array}{c}2.1(24) \\
43.4(486) \\
5.6(63) \\
24.9(279) \\
23.9(267\end{array}$ & $\begin{aligned} 2.2 & (20) \\
44.6 & (413) \\
6.2 & (57) \\
24.5 & (227) \\
22.5 & (208)\end{aligned}$ & $\begin{array}{c}2.1(24) \\
43.4(486) \\
5.6(63) \\
24.9(279) \\
23.9(267)\end{array}$ \\
\hline Spinal Surgeries $[\%(n)]$ & $9.5(451)$ & $20.9(234)$ & $25.3(234)$ & $21.2(234)$ \\
\hline Other Musculoskeletal Surgeries [\% (n)] & $39.1(1847)$ & $21.8(244)$ & $26.3(244)$ & $22.1(244)$ \\
\hline
\end{tabular}


Table A.34 - Continued

\begin{tabular}{|l|c|c|c|c|}
\hline Length of Disability (SD) & $44.6(37.3)$ & $45.5(35.2)$ & $38.3(31.9)$ & $18.7(21.0)^{*}$ \\
$\quad 4-8$ months [\% (n)] & $21.5(1015)$ & $17.8(199)$ & $21.5(199)$ & $33.3(373)$ \\
$9-18$ months [\% (n)] & $13.8(654)$ & $13.0(145)$ & $15.7(145)$ & $33.3(373)$ \\
$>18$ months [\% (n)] & $64.7(3057)$ & $69.3(775)$ & $62.8(581)$ & $33.3(373)$ \\
& & & & $4-266$ \\
Range (months) & $4-132$ & $4-132$ & $4-132$ & $0.5(6)$ \\
\hline$\%$ PRIDE $>132$ months [\% (n)] & - & - & - & \\
\hline
\end{tabular}

* Significantly different $(\mathrm{p}<.05)$ compared to the OBWC Final Matched group 
Table A.35. Percentiles of Total Medical Cost in the initial match of the OBWC Cohort $(\mathrm{N}=1119)$

\begin{tabular}{|l|rrrrrrr|}
\hline Disability Duration & \multicolumn{7}{|c|}{ Percentiles of Total Medical Cost } \\
\cline { 2 - 8 } Groups & 5 th & 10 th & 25 th & 50 th & 75 th & 90 th & 95 th \\
\hline 4 - 8 months & $\$ 1,276$ & $\$ 3,064$ & $\$ 6,924$ & $\$ 13,705$ & $\$ 27,275$ & $\$ 46,651$ & $\$ 67,876$ \\
9 - 17 months & $\$ 4,984$ & $\$ 8,089$ & $\$ 16,790$ & $\$ 27,123$ & $\$ 42,521$ & $\$ 67,885$ & $\$ 88,431$ \\
$>18$ months & $\$ 163$ & $\$ 184$ & $\$ 890$ & $\$ 33,153$ & $\$ 73,516$ & $\$ 127,003$ & $\$ 151,303$ \\
& & & & & & & \\
\hline
\end{tabular}


Table A.36. Correlations among Medical Cost, Log Medical Cost, Age, and Gender in the final OBWC Cohort $(\mathrm{N}=925)$

\begin{tabular}{lccc}
\hline & Medical Cost & Log Medical Cost & Age \\
\hline Medical Cost & 1 & & \\
Log Medical Cost & $.70^{*}$ & 1 & 1 \\
Age & -.02 & $<.01$ & -.06 \\
Gender & .04 & .01 & \\
\hline
\end{tabular}

* Significant at $p<.05$

$\bar{a}$ 
Table A.37. Estimated Total Medical Costs and Costs by Major Provider and Service Types $(\mathrm{N}=925)$

\begin{tabular}{|c|c|c|c|c|c|}
\hline Variables & $\begin{array}{c}\text { OBWC } \\
4-8 \text { months } \\
\mathrm{N}=199\end{array}$ & $\begin{array}{c}\text { OBWC } \\
9-18 \text { months } \\
\mathrm{N}=145\end{array}$ & $\begin{aligned} & \text { OBWC } \\
> & 18 \text { months } \\
& \mathrm{N}=581\end{aligned}$ & $\begin{array}{c}\mathrm{p} \text {-values } \\
\text { group diff/lin. trend }\end{array}$ & $\begin{array}{c}\text { Effect Size } \\
\eta^{2}\end{array}$ \\
\hline $\begin{array}{l}\text { Physician } \\
\text { N } \\
\text { Median \$ (IQR) } \\
\text { \$ (SD) } \\
\text { Log \$ (SD) }\end{array}$ & $\begin{array}{c}198 \\
\$ 3,619(\$ 5,349) \\
\$ 5,457(\$ 5,432) \\
8.2(1.0)\end{array}$ & $\begin{array}{c}145 \\
\$ 5,887(\$ 6,452) \\
\$ 8,071(\$ 7,940) \\
8.6(1.0)^{*}\end{array}$ & $\begin{array}{c}580 \\
\$ 12,276(\$ 15,036) \\
\$ 16,057(\$ 14,277) \\
9.3(1.1)^{*}\end{array}$ & $<.001 /<.001$ & .161 \\
\hline $\begin{array}{l}\text { Pharmacy } \\
\text { N } \\
\text { Median \$ (IQR) } \\
\text { \$ (SD) } \\
\text { Log \$ (SD) }\end{array}$ & $\begin{array}{c}131 \\
\$ 481(\$ 1736) \\
\$ 2,270(\$ 4,392) \\
6.1(2.0)\end{array}$ & $\begin{array}{c}116 \\
\$ 741(\$ 3,083) \\
\$ 4,284(\$ 8,491) \\
6.7(1.9)^{*}\end{array}$ & $\begin{array}{c}510 \\
\$ 5,079(\$ 13,794) \\
\$ 11,818(\$ 17,640) \\
8.2(1.9)^{*}\end{array}$ & $<.001 /<.001$ & .163 \\
\hline $\begin{array}{l}\text { Case Management } \\
\text { N } \\
\text { Median \$ (IQR) } \\
\text { \$ (SD) } \\
\text { Log \$ (SD) }\end{array}$ & $\begin{array}{c}63 \\
\$ 1,689(\$ 2,186) \\
\$ 1,924(\$ 1,605) \\
7.2(0.9)\end{array}$ & $\begin{array}{c}83 \\
\$ 2,903(\$ 4,534) \\
\$ 3,761(\$ 3,360) \\
7.8(1.1)^{*}\end{array}$ & $\begin{array}{c}384 \\
\$ 3,645(\$ 5,434) \\
\$ 4,767(\$ 4,671) \\
10.0(1.1)^{*}\end{array}$ & $<.001 /<.001$ & .047 \\
\hline
\end{tabular}


Table A.37 - Continued

\begin{tabular}{|c|c|c|c|c|c|}
\hline $\begin{array}{l}\text { Hospital } \\
\text { N } \\
\text { Median \$ (IQR) } \\
\text { \$ (SD) } \\
\text { Log \$ (SD) }\end{array}$ & $\begin{array}{c}174 \\
\$ 4,986(\$ 9,376) \\
\$ 10,203(\$ 14,189) \\
8.4(1.5)\end{array}$ & $\begin{array}{c}131 \\
\$ 7,102(\$ 13,537) \\
\$ 11,824(\$ 14,367) \\
8.6(1.5)\end{array}$ & $\begin{array}{c}542 \\
\$ 13,041(\$ 27,879) \\
\$ 26,526(\$ 48,396) \\
9.2(1.6)^{*}\end{array}$ & $<.001 /<.001$ & .052 \\
\hline $\begin{array}{l}\text { Psychologists \& } \\
\text { Licensed Profession } \\
\text { Counselors } \\
\text { N } \\
\text { Median \$ (IQR) } \\
\text { \$(SD) } \\
\text { Log \$ (SD) }\end{array}$ & $\begin{array}{c}12 \\
\$ 429(\$ 1,363) \\
\$ 1,658(\$ 3,581) \\
6.3(1.4)\end{array}$ & $\begin{array}{c}11 \\
\$ 1,255(\$ 2,099) \\
\$ 1,727(\$ 1,586) \\
7.0(1.0)\end{array}$ & $\begin{array}{c}222 \\
\$ 2,110(\$ 4,322) \\
\$ 3,414(\$ 3,922) \\
7.4(1.4)^{*}\end{array}$ & $.019 / .006$ & .032 \\
\hline $\begin{array}{l}\text { Physical/Occupatiol } \\
\text { Therapists } \\
\text { N } \\
\text { Median \$ (IQR) } \\
\text { \$ (SD) } \\
\text { Log \$ (SD) }\end{array}$ & $\begin{array}{c}100 \\
\$ 2,028(\$ 3,600) \\
\$ 3,231(\$ 3,405) \\
7.5(1.2)\end{array}$ & $\begin{array}{c}88 \\
\$ 4,352(\$ 6,884) \\
\$ 5,808(\$ 5,859) \\
8.1(1.2)^{*}\end{array}$ & $\begin{array}{c}396 \\
\$ 2,849(\$ 5,808) \\
\$ 4,970(\$ 6,276) \\
7.8(1.3)\end{array}$ & $<.002 / .029$ & .021 \\
\hline $\begin{array}{l}\text { Chiropractor } \\
\text { N } \\
\text { Median \$ (IQR) } \\
\text { \$ (SD) } \\
\text { Log \$ (SD) }\end{array}$ & $\begin{array}{c}49 \\
\$ 5,247(\$ 9,653) \\
\$ 8,074(\$ 11,657) \\
8.1(1.6)\end{array}$ & $\begin{array}{c}52 \\
\$ 5,037(\$ 12,800) \\
\$ 10,375(\$ 15,395) \\
8.2(1.7)\end{array}$ & $\begin{array}{c}233 \\
\$ 4,471(\$ 9,099) \\
\$ 8,678(\$ 12,290) \\
8.1(1.6)\end{array}$ & $.909 / .833$ & \\
\hline
\end{tabular}


Table A.37 - Continued

\begin{tabular}{|c|c|c|c|c|c|}
\hline $\begin{array}{l}\text { Pain Management } \\
\text { Centers } \\
\text { N } \\
\text { Median \$ (IQR) } \\
\text { \$ (SD) } \\
\text { Log \$ (SD) }\end{array}$ & $\begin{array}{c}0 \\
\text { N/A } \\
\text { N/A } \\
\text { N/A }\end{array}$ & $\begin{array}{c}4 \\
\$ 4,244(\$ 12,800) \\
\$ 6,496(\$ 7,103) \\
8.2(1.3)\end{array}$ & $\begin{array}{c}18 \\
\$ 2,997(\$ 10,456) \\
\$ 6,490(\$ 8,493) \\
7.6(1.9)\end{array}$ & .535 & \\
\hline $\begin{array}{l}\text { Job Retraining } \\
\text { N } \\
\text { Median \$ (IQR) } \\
\text { \$ (SD) } \\
\text { Log \$ (SD) }\end{array}$ & $\begin{array}{c}11 \\
\$ 730(\$ 1861) \\
\$ 2,063(\$ 3,109) \\
6.8(1.3)\end{array}$ & $\begin{array}{c}22 \\
\$ 1,193(\$ 1,464) \\
\$ 1,668(\$ 1,287) \\
7.2(0.7)\end{array}$ & $\begin{array}{c}133 \\
\$ 2,370(\$ 3,133) \\
\$ 3,041(\$ 2,969) \\
7.5(1.1)\end{array}$ & $.050 / .036$ & \\
\hline $\begin{array}{l}\text { Other Providers } \\
\text { N } \\
\text { Median \$ (IQR) } \\
\text { \$ (SD) } \\
\text { Log \$ (SD) }\end{array}$ & $\begin{array}{c}137 \\
\$ 835(\$ 1675) \\
\$ 1,561(\$ 2,126) \\
6.6(1.3)\end{array}$ & $\begin{array}{c}105 \\
\$ 1,129(\$ 1,955) \\
\$ 2,220(\$ 3,511) \\
7.0(1.3)\end{array}$ & $\begin{array}{c}477 \\
\$ 2,332(\$ 4,186) \\
\$ 5,170(\$ 19,059) \\
7.6(1.4)^{*}\end{array}$ & $<.001 /<.001$ & .081 \\
\hline
\end{tabular}

* Significantly different compared to ER group, at $p<.05$ after adjusting for inflation of Type I error 
Table A.38. Effect Sizes for Post-Hoc Comparisons of Significant Differences in Medical Costs $(\mathrm{N}=925)$

\begin{tabular}{|l|c|c|}
\hline \multirow{2}{*}{ Variables } & \multicolumn{2}{|c|}{ Cohen's $d$} \\
\cline { 2 - 3 } & ID vs. ER & DR vs. ER \\
\hline Total Medical Cost & 0.6 & 1.1 \\
\hline Physician & 0.4 & 1.0 \\
\hline Chiropractor & NS & NS \\
\hline Hospital & NS & 0.5 \\
\hline Psychological Services & NS & 0.8 \\
\hline Physical/Occupational Therapists & 0.5 & NS \\
\hline Pain Management & NS & NS \\
\hline Case Management & 0.5 & 0.7 \\
\hline Job Retraining & NS & NS \\
\hline Pharmacy & 0.3 & 0.7 \\
\hline Other Service Providers & NS & \\
\hline
\end{tabular}


Table A.39. Estimated Total Medical Costs by Major Procedures and Interventions, based on the OBWC Cohort $(\mathrm{N}=925)$

\begin{tabular}{|c|c|c|c|c|c|}
\hline Variables & $\begin{array}{c}\text { OBWC } \\
4-8 \text { months } \\
\mathrm{N}=199\end{array}$ & $\begin{array}{c}\text { OBWC } \\
9-18 \text { months } \\
\mathrm{N}=145\end{array}$ & $\begin{array}{c}\text { OBWC } \\
>18 \text { months } \\
\mathrm{N}=581\end{array}$ & $\begin{array}{c}\text { p-values } \\
\text { group diff/lin. trend }\end{array}$ & $\begin{array}{c}\text { Effect Size } \\
\eta^{2}\end{array}$ \\
\hline $\begin{array}{l}\text { Consultation, Evaluation \& } \\
\text { Management } \\
\text { N } \\
\text { Median \$ (IQR) } \\
\text { \$ (SD) } \\
\text { Log \$ (SD) }\end{array}$ & $\begin{array}{c}193 \\
\$ 822(\$ 1,083) \\
\$ 1,196(\$ 1,251) \\
6.7(0.9)\end{array}$ & $\begin{array}{c}145 \\
\$ 1,509(\$ 1,580) \\
\$ 1,954(\$ 1,865) \\
7.2(0.9)^{*}\end{array}$ & $\begin{array}{c}576 \\
\$ 3,058(\$ 3,530) \\
\$ 3,728(\$ 2,832) \\
7.9(0.9)^{*}\end{array}$ & $<.001 /<.001$ & .225 \\
\hline $\begin{array}{l}\text { Medication } \\
\quad \mathrm{N} \\
\text { Median \$ (IQR) } \\
\text { \$ (SD) } \\
\text { Log \$ (SD) }\end{array}$ & $\begin{array}{c}130 \\
\$ 474(\$ 1741) \\
\$ 2,275(\$ 4,403) \\
6.1(2.0)\end{array}$ & $\begin{array}{c}116 \\
\$ 741(\$ 3,083) \\
\$ 4,281(\$ 8,492) \\
6.7(1.9)^{*}\end{array}$ & $\begin{array}{c}508 \\
\$ 5,029(\$ 13,828) \\
\$ 11,802(\$ 17,651) \\
8.2(1.9)^{*}\end{array}$ & $<.001 /<.001$ & .159 \\
\hline $\begin{array}{l}\text { Diagnostics } \\
\quad \mathrm{N} \\
\text { Median \$ (IQR) } \\
\text { Mean \$ (SD) } \\
\text { Log \$ (SD) }\end{array}$ & $\begin{array}{c}180 \\
\$ 656(\$ 832) \\
\$ 859(\$ 875) \\
6.2(1.3)\end{array}$ & $\begin{array}{c}138 \\
\$ 894(\$ 1,403) \\
\$ 1,214(\$ 1,176) \\
6.6(1.1)^{*}\end{array}$ & $\begin{array}{c}555 \\
\$ 1,751(\$ 2,541) \\
\$ 2,482(\$ 2,618) \\
7.2(1.3)^{*}\end{array}$ & $<. .001 /<.001$ & .100 \\
\hline $\begin{array}{l}\text { Injection Therapeutics } \\
\mathrm{N} \\
\text { Median } \$(\mathrm{IQR}) \\
\text { \$ (SD) } \\
\text { Log \$ (SD) }\end{array}$ & $\begin{array}{c}80 \\
\$ 249(\$ 531) \\
\$ 614(\$ 1,006) \\
5.6(1.4)\end{array}$ & $\begin{array}{c}76 \\
\$ 369(\$ 839) \\
\$ 1,109(\$ 3,429) \\
5.9(1.4)\end{array}$ & $\begin{array}{c}380 \\
\$ 905(\$ 1,772) \\
\$ 2,278(\$ 6,160) \\
6.7(1.5)^{*}\end{array}$ & $<.001 /<.001$ & .080 \\
\hline
\end{tabular}


Table A.39 - Continued

\begin{tabular}{|c|c|c|c|c|c|}
\hline $\begin{array}{l}\text { Anesthesia } \\
\text { N } \\
\text { Median \$ (IQR) } \\
\text { \$ (SD) } \\
\text { Log \$ (SD) }\end{array}$ & $\begin{array}{c}92 \\
\$ 661(\$ 534) \\
\$ 774(\$ 558) \\
6.4(0.6)\end{array}$ & $\begin{array}{c}72 \\
\$ 713(\$ 658) \\
\$ 919(\$ 746) \\
6.5(0.8)\end{array}$ & $\begin{array}{c}339 \\
\$ 961(\$ 985) \\
\$ 1,298(\$ 1,196) \\
6.8(0.8)^{*}\end{array}$ & $<.001 /<.001$ & .044 \\
\hline $\begin{array}{l}\text { Spine Surgeries } \\
\text { N } \\
\text { Median \$ (IQR) } \\
\text { \$ (SD) } \\
\text { Log \$ (SD) }\end{array}$ & $\begin{array}{c}26 \\
\$ 3,625(\$ 5,139) \\
\$ 5,355(\$ 4,318) \\
8.2(0.9)\end{array}$ & $\begin{array}{c}20 \\
\$ 3,792(\$ 5,170) \\
\$ 5,447(\$ 4,136) \\
8.3(0.8)\end{array}$ & $\begin{array}{c}188 \\
\$ 5,744(\$ 7,556) \\
\$ 7,146(\$ 5,652) \\
8.5(0.9)\end{array}$ & $.182 / .103$ & \\
\hline $\begin{array}{l}\text { Other Musculoskeletal } \\
\text { Surgeries } \\
\text { N } \\
\text { Median \$ (IQR) } \\
\text { \$ (SD) } \\
\text { Log \$ (SD) }\end{array}$ & $\begin{array}{c}67 \\
\$ 1,958(\$ 2,196) \\
\$ 2,679(\$ 2,014) \\
7.6(0.7)\end{array}$ & $\begin{array}{c}48 \\
\$ 2,525(\$ 3,299) \\
\$ 3,971(\$ 3,634) \\
7.9(0.9)\end{array}$ & $\begin{array}{c}127 \\
\$ 2,705(\$ 3,788) \\
\$ 3,660(\$ 3,240) \\
7.8(0.9)\end{array}$ & $.214 / .190$ & \\
\hline $\begin{array}{l}\text { PM\&R Modalities } \\
\text { N } \\
\text { Median \$ (IQR) } \\
\text { \$ (SD) } \\
\text { Log \$ (SD) }\end{array}$ & $\begin{array}{c}85 \\
\$ 1,603(\$ 3,435) \\
\$ 2,929(\$ 3,039) \\
7.4(1.2)\end{array}$ & $\begin{array}{c}79 \\
\$ 3,106(\$ 4,316) \\
\$ 4,558(\$ 5,450) \\
7.9(1.2)\end{array}$ & $\begin{array}{c}377 \\
\$ 2,325(\$ 3,180) \\
\$ 4,574(\$ 5,809) \\
7.7(1.5)\end{array}$ & $.138 / .138$ & \\
\hline
\end{tabular}


Table A.39 - Continued

\begin{tabular}{|l|c|c|c|c|}
\hline Work & & & & \\
Hardening/Conditioning & 16 & 45 & 129 & \\
$\mathrm{~N}$ & $\$ 1,163(\$ 3,137)$ & $\$ 3,276(\$ 6,095)$ & $\$ 2,325(\$ 3,180)$ & \\
Median \$(IQR) & $\$ 3,093(\$ 4,378)$ & $\$ 4,847(\$ 5,019)$ & $\$ 3,737(\$ 4,238)$ & \\
\$(SD) & $7.4(1.1)$ & $7.9(1.2)$ & $7.7(1.1)$ & $.264 / .370$ \\
Log \$(SD) & & & & \\
\hline
\end{tabular}

* Significantly different compared to ER group, at $p<.05$ after adjusting for inflation of Type I error 
Table A.40. Effect Sizes for Post-Hoc Comparisons of Significant Differences in Costs of Specific Medical Procedures and Interventions $(\mathrm{N}=925)$

\begin{tabular}{|l|c|c|}
\hline \multirow{2}{*}{ Variables } & \multicolumn{2}{|c|}{ Cohen's $d$} \\
\cline { 2 - 3 } & ID vs. ER & DR vs. ER \\
\hline $\begin{array}{l}\text { Consultation, Evaluation \& } \\
\text { Management }\end{array}$ & 0.6 & 1.3 \\
\hline Medication & 0.3 & 1.1 \\
\hline Diagnostics & 0.3 & 0.8 \\
\hline Injection Therapeutics & NS & 0.7 \\
\hline Anesthesia & NS & 0.5 \\
\hline
\end{tabular}


Table A.41. Estimated Productivity Losses and Accrued Disability Benefits $(\mathrm{N}=1119)$

\begin{tabular}{|c|c|c|c|c|c|}
\hline Variables & $\begin{array}{c}\text { ER } \\
N=373\end{array}$ & $\begin{array}{c}\text { ID } \\
N=373\end{array}$ & $\begin{array}{c}\text { DR } \\
N=373\end{array}$ & $\begin{array}{c}\text { p-values } \\
\text { group diff/lin. trend }\end{array}$ & $\begin{array}{c}\text { Effect Size } \\
\eta^{2}\end{array}$ \\
\hline $\begin{array}{l}\text { Productivity Losses } \\
\text { N } \\
\text { Median (IQR) } \\
\text { \$ (SD) } \\
\text { Log \$ (SD) }\end{array}$ & $\begin{array}{c}358 \\
\$ 10,656(\$ 11,923) \\
\$ 12,547(\$ 13,604) \\
9.3(0.8)\end{array}$ & $\begin{array}{c}364 \\
\$ 26,177(\$ 25,302) \\
\$ 32,510(\$ 33,465) \\
10.1(0.8)^{*}\end{array}$ & $\begin{array}{c}355 \\
\$ 50,963(\$ 60,800) \\
\$ 73,075(\$ 66,289) \\
10.9(0.9)^{*}\end{array}$ & $<.001 /<.001$ & 0.365 \\
\hline $\begin{array}{l}\text { Total Disability Benefits } \\
\text { N } \\
\text { Median (IQR) } \\
\text { \$ (SD) } \\
\text { Log \$ (SD) }\end{array}$ & $\begin{array}{c}337 \\
\$ 6,202(\$ 6,556) \\
\$ 7,328(\$ 4,776) \\
8.7(0.8)\end{array}$ & $\begin{array}{c}356 \\
\$ 17,056(\$ 13,827) \\
\$ 18,411(\$ 10,270) \\
9.6(0.7)^{*}\end{array}$ & $\begin{array}{c}348 \\
\$ 32,341(\$ 29,964) \\
\$ 36,790(\$ 25,028) \\
10.3(0.8)^{*}\end{array}$ & $<.001 /<.001$ & 0.438 \\
\hline $\begin{array}{l}\text { FECA - TTD Benefits } \\
\text { N } \\
\text { Median (IQR) } \\
\text { \$ (SD) } \\
\text { Log \$ (SD) }\end{array}$ & $\begin{array}{c}7 \\
\$ 6,813(\$ 7,087) \\
\$ 6,892(\$ 3,170) \\
8.7(0.5)\end{array}$ & $\begin{array}{c}9 \\
\$ 24,129(\$ 17,662) \\
\$ 22,051(\$ 10,646) \\
9.9(0.6)^{*}\end{array}$ & $\begin{array}{c}20 \\
\$ 37,290(\$ 40,635) \\
\$ 45,897(\$ 46,106) \\
10.3(1.0)^{*}\end{array}$ & $.001 /<.001$ & 0.344 \\
\hline
\end{tabular}


Table A.41 - Continued

\begin{tabular}{|c|c|c|c|c|c|}
\hline $\begin{array}{l}\text { Non-Subscriber - TTD } \\
\text { Benefits } \\
\text { N } \\
\text { Median (IQR) } \\
\text { \$ (SD) } \\
\text { Log \$ (SD) }\end{array}$ & $\begin{array}{c}10 \\
\$ 5,562(\$ 6,594) \\
\$ 6,263(\$ 3,475) \\
8.5(0.7)\end{array}$ & $\begin{array}{c}12 \\
\$ 13,397(\$ 10,761) \\
\$ 14,281(\$ 8,154) \\
9.4(0.6)^{*}\end{array}$ & $\begin{array}{c}3 \\
\$ 32,964(\$ 7,540) \\
\$ 31,520(\$ 3,972) \\
10.4(0.1)^{*}\end{array}$ & $.001 /<.001$ & 0.498 \\
\hline $\begin{array}{l}\text { Out of State - TTD } \\
\text { Benefits } \\
\text { N } \\
\text { Median (IQR) } \\
\text { \$ (SD) } \\
\text { Log \$ (SD) }\end{array}$ & $\begin{array}{c}10 \\
\$ 7,032(\$ 6,613) \\
\$ 7,576(\$ 3,583) \\
8.8(0.6)\end{array}$ & $\begin{array}{c}2 \\
\$ 8,626(\$ 13,838) \\
\$ 8,625(\$ 9,784) \\
8.5(1.6)\end{array}$ & $\begin{array}{c}10 \\
\$ 53,426(\$ 66,652) \\
\$ 64,751(\$ 55,683) \\
10.8(0.8)^{*}\end{array}$ & $.001 / .001$ & 0.638 \\
\hline $\begin{array}{l}\text { Impairment Income } \\
\text { Benefits } \\
\text { N } \\
\text { Median (IQR) } \\
\text { \$ (SD) } \\
\text { Log \$ (SD) }\end{array}$ & $\begin{array}{c}5 \\
\$ 7,825(\$ 2,650) \\
\$ 8,290(\$ 3,324) \\
8.9(0.4)\end{array}$ & $\begin{array}{c}61 \\
\$ 8,616(\$ 5,809) \\
\$ 9,336(\$ 4,640) \\
9.0(0.5)\end{array}$ & $\begin{array}{c}139 \\
\$ 13,026(\$ 9,709) \\
\$ 14,839(\$ 8,820) \\
9.4(0.6)^{*}\end{array}$ & $<.001 /<.001$ & 0.124 \\
\hline
\end{tabular}

* Significantly different compared to ER group, at $p<.05$ after adjusting for inflation of Type I error 
Table A.42. Effect Sizes for Post-Hoc Comparisons of Productivity Losses and Disability Benefits ( $N=1119)$

\begin{tabular}{|l|c|c|}
\hline \multirow{2}{*}{ Variables } & \multicolumn{2}{|c|}{ Cohen's $d$} \\
\cline { 2 - 3 } & ID vs. ER & DR vs. ER \\
\hline Productivity Losses & 1.0 & 1.9 \\
\hline Total Disability Benefits & 1.3 & 2.2 \\
\hline Texas WC - TIBS & 1.2 & 1.9 \\
\hline FECA - TTD Benefits & 1.3 & 1.8 \\
\hline Non-Subscriber - TTD Benefits & 1.4 & 2.8 \\
\hline Out of State - TTD Benefits & NS & 2.3 \\
\hline Impairment Income Benefits & NS & 0.9 \\
\hline
\end{tabular}


Table A.43. Potential Savings in Costs over the Duration of Disability

\begin{tabular}{|c|c|c|c|}
\hline Savings over Duration of Disability & ER & ID & DR \\
\hline Medical Costs & $\$ 21,356$ & $\$ 33,750$ & $\$ 67,612$ \\
\hline Average Savings & - & $\$ 12,394$ & $\$ 46,256$ \\
\hline$\%$ Saved & - & $37 \%$ & $68 \%$ \\
\hline Disability Benefits & $\$ 7,328$ & $\$ 18,411$ & $\$ 36,790$ \\
\hline Average Savings & - & $\$ 11,083$ & $\$ 29,462$ \\
\hline$\%$ Saved & - & $60 \%$ & $80 \%$ \\
\hline Productivity Losses & $\$ 12,547$ & $\$ 32,510$ & $\$ 73,075$ \\
\hline Average Savings & - & $\$ 19,963$ & $\$ 60,528$ \\
\hline$\%$ Saved & - & $61 \%$ & $83 \%$ \\
\hline Analytical Timeframe (Program + One-Year) & $\$ 29,255$ & $\$ 30,700$ & $\$ 30,553$ \\
\hline Average Savings & - & $\$ 1,445$ & $\$ 1,298$ \\
\hline$\%$ Saved & - & $5 \%$ & $4 \%$ \\
\hline Total Cost of Illness & $\$ 70,486$ & $\$ 115,371$ & $\$ 208,030$ \\
\hline Average Savings & - & $\$ 44,885$ & $\$ 137,544$ \\
\hline$\%$ Saved & - & $39 \%$ & $66 \%$ \\
\hline
\end{tabular}


APPENDIX B

FIGURES 


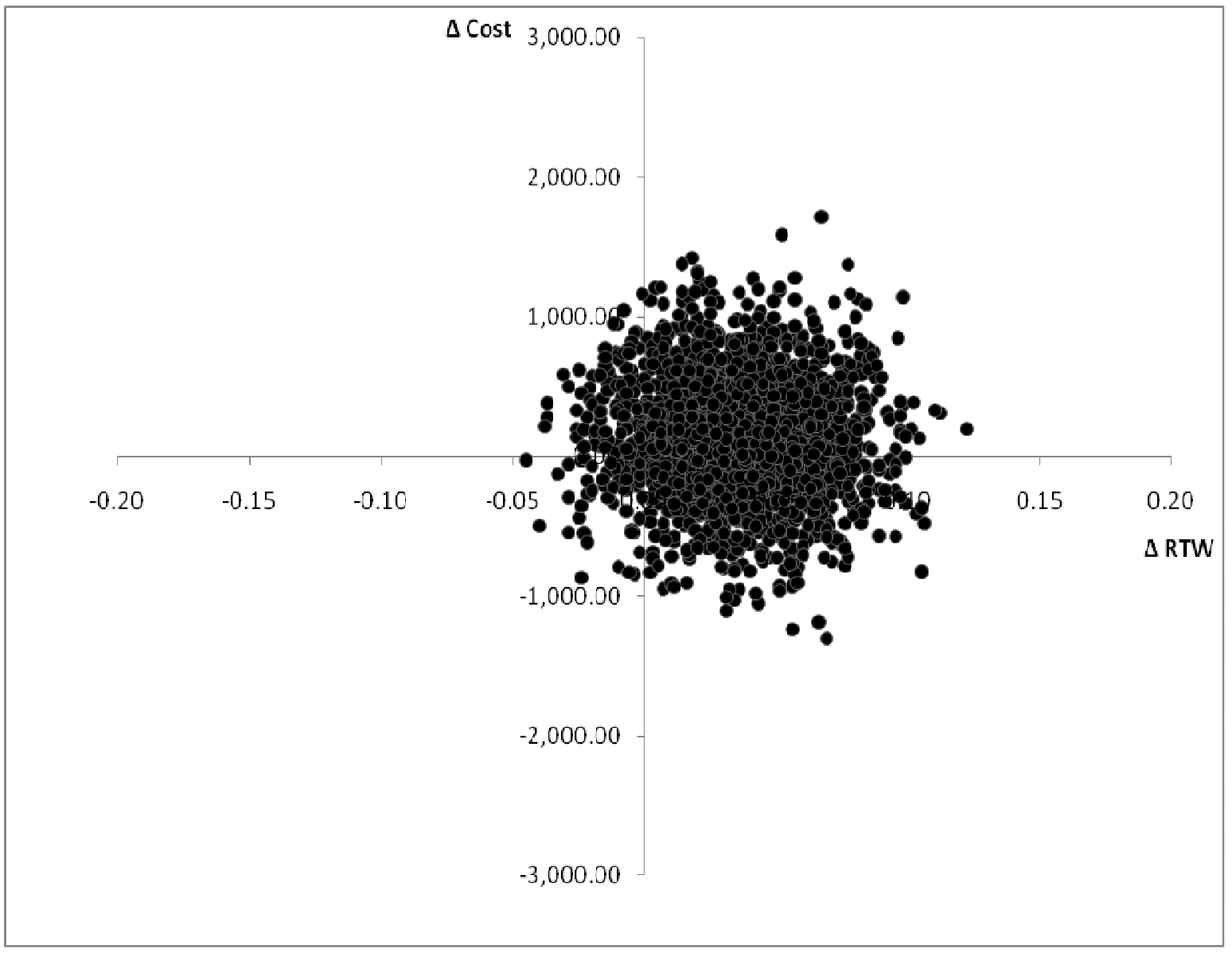

Figure B.1. Incremental Cost-Effectiveness Plane Comparing the ID and DR Groups on the Outcome of RTW at One-Year 


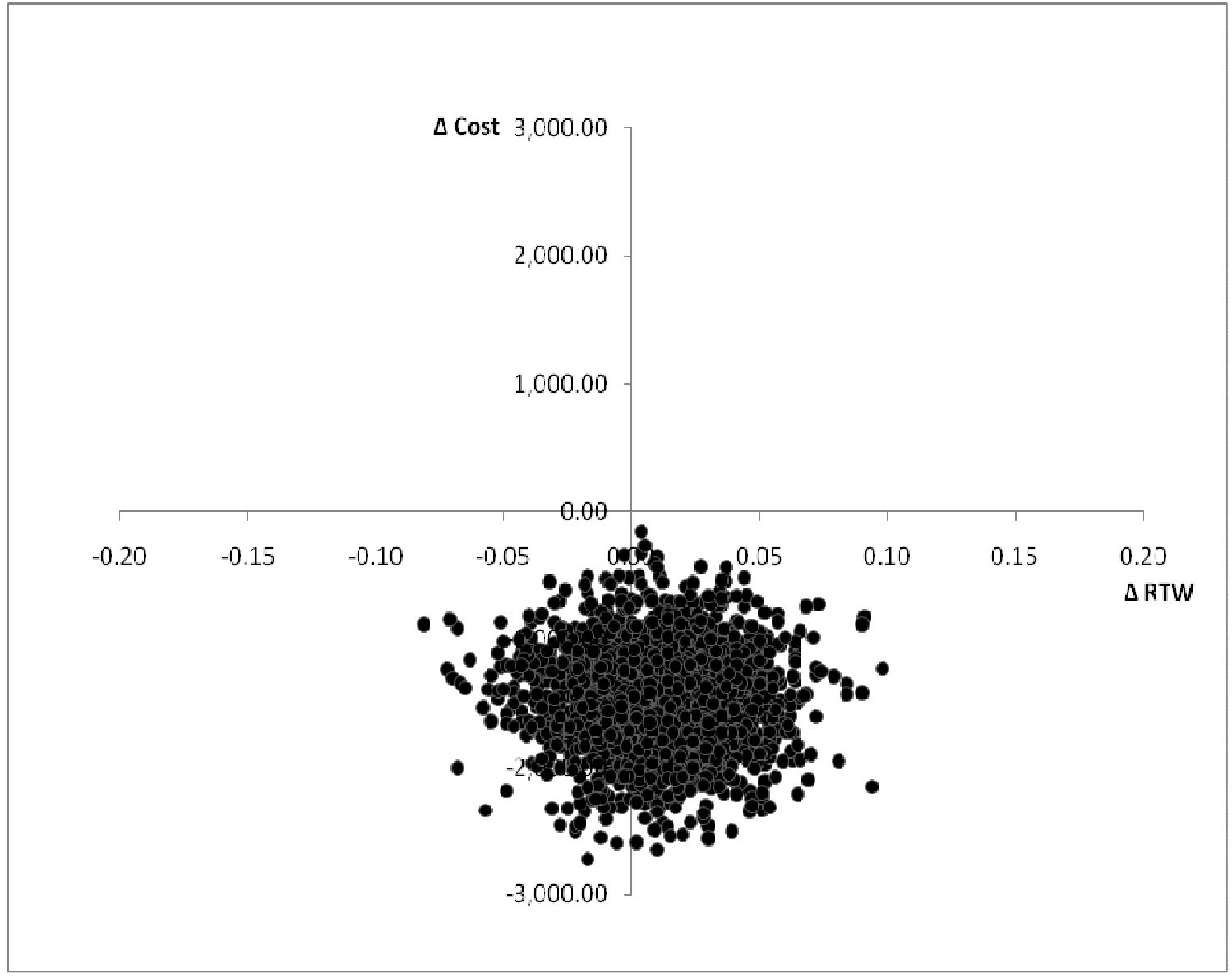

Figure B.2. Incremental Cost-Effectiveness Plane Comparing the ER and ID Groups on the Outcome of RTW at One-Year 


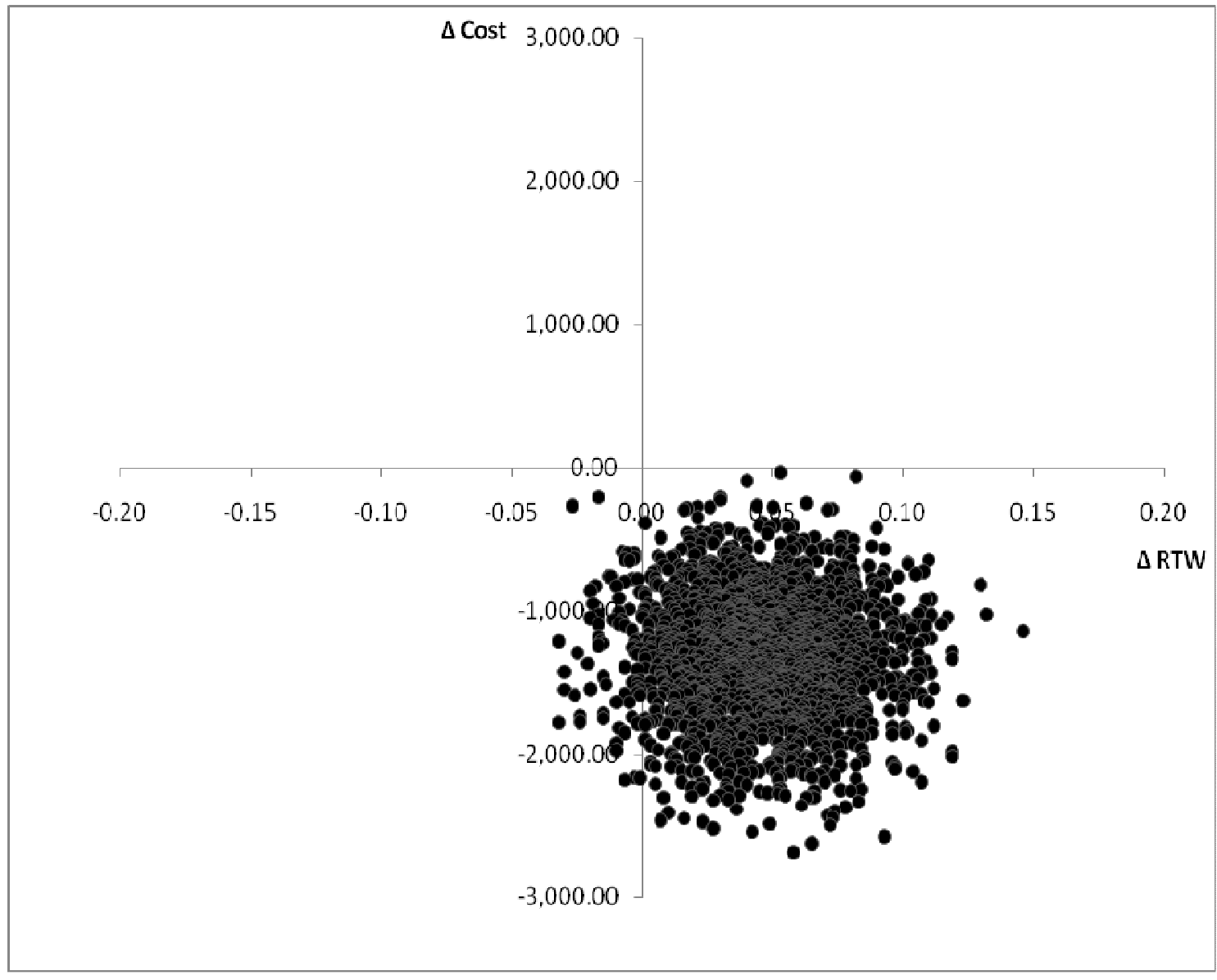

Figure B.3. Incremental Cost-Effectiveness Plane Comparing the ER and DR Groups on the Outcome of RTW at One-Year 


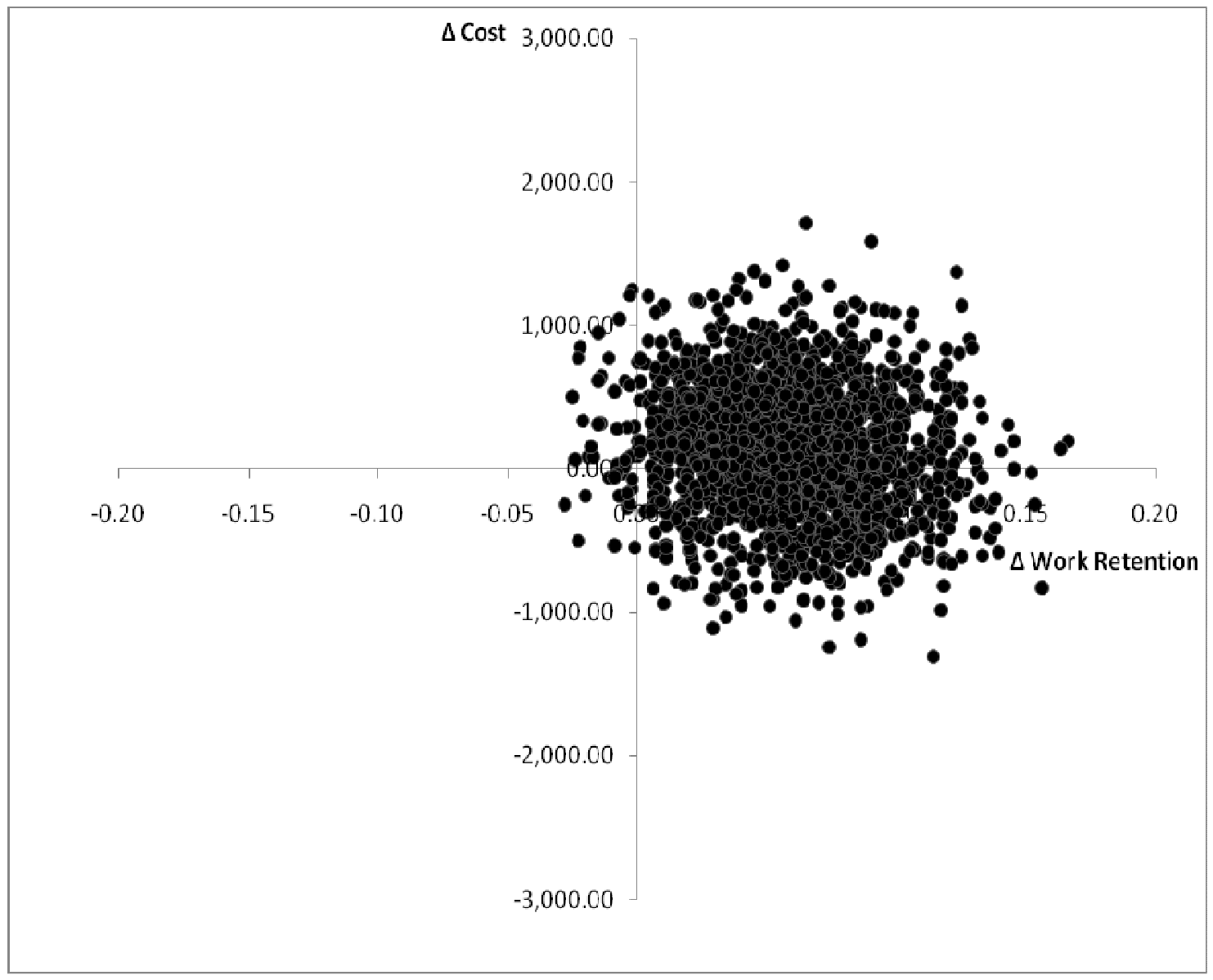

Figure B.4. Incremental Cost-Effectiveness Plane Comparing the ID and DR Groups on the Outcome of Work Retention at One-Year 


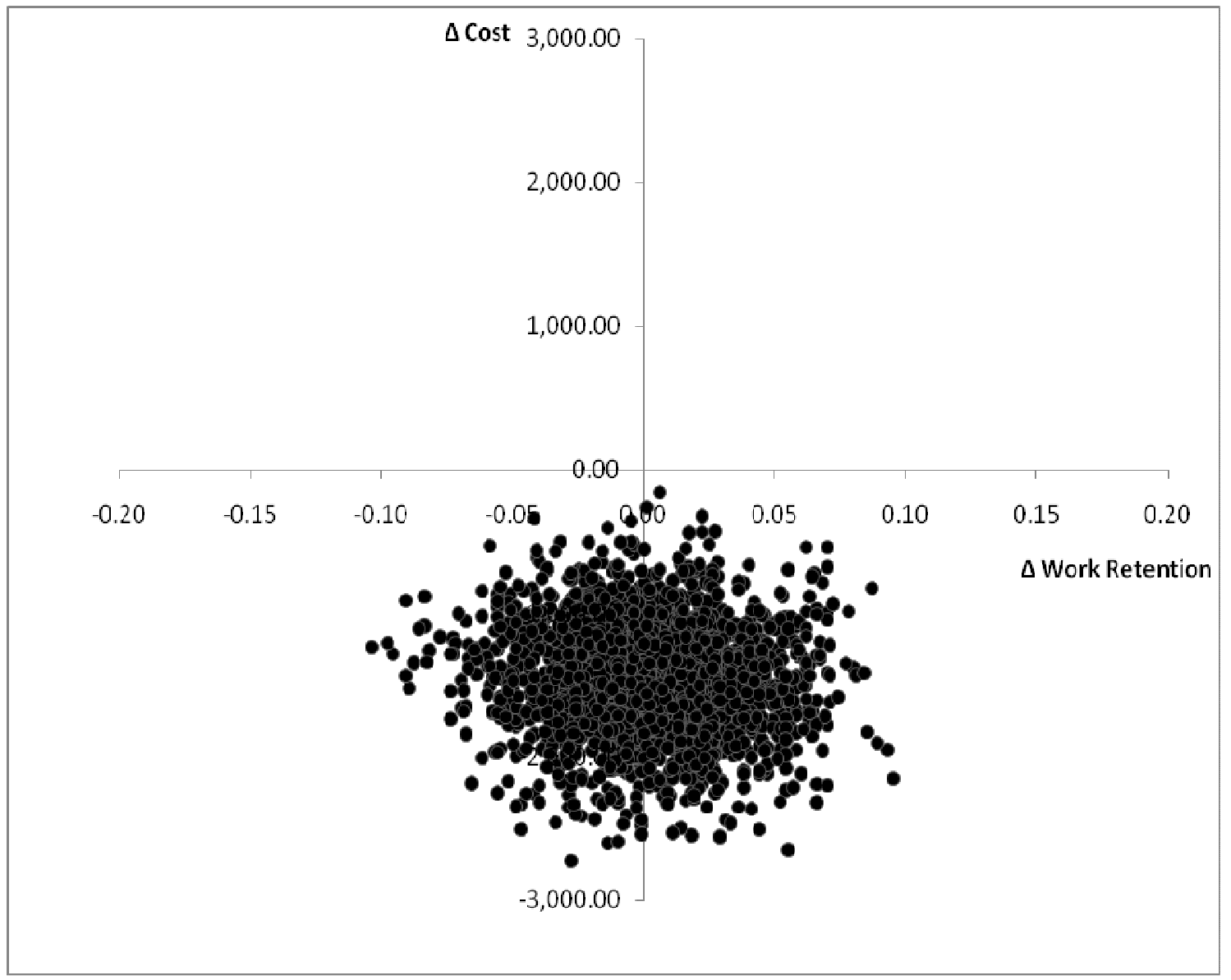

Figure B.5. Incremental Cost-Effectiveness Plane Comparing the ER and ID Groups on the Outcome of Work Retention at One-Year 


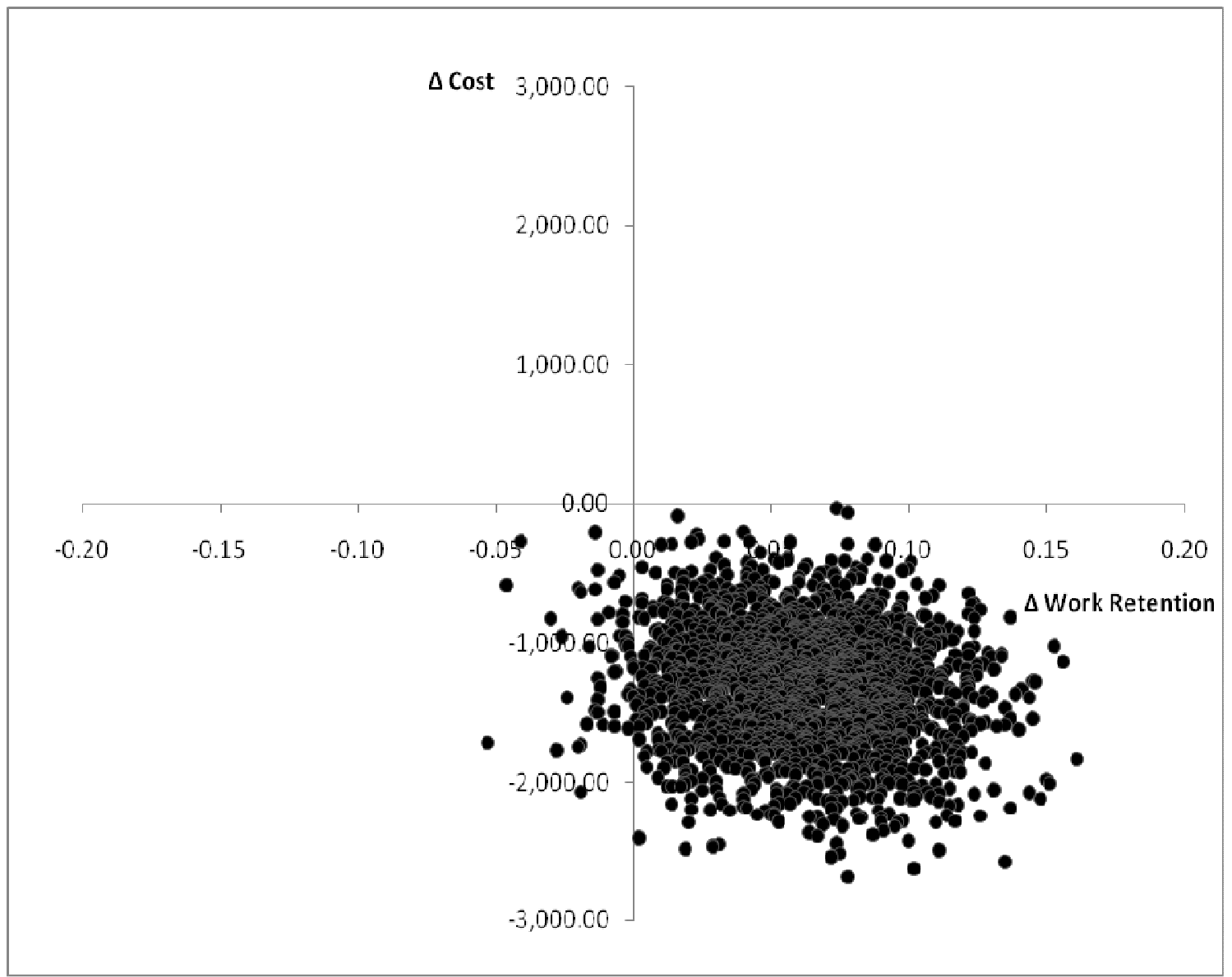

Figure B.6. Incremental Cost-Effectiveness Plane Comparing the ER and DR Groups on the Outcome of Work Retention at One-Year 


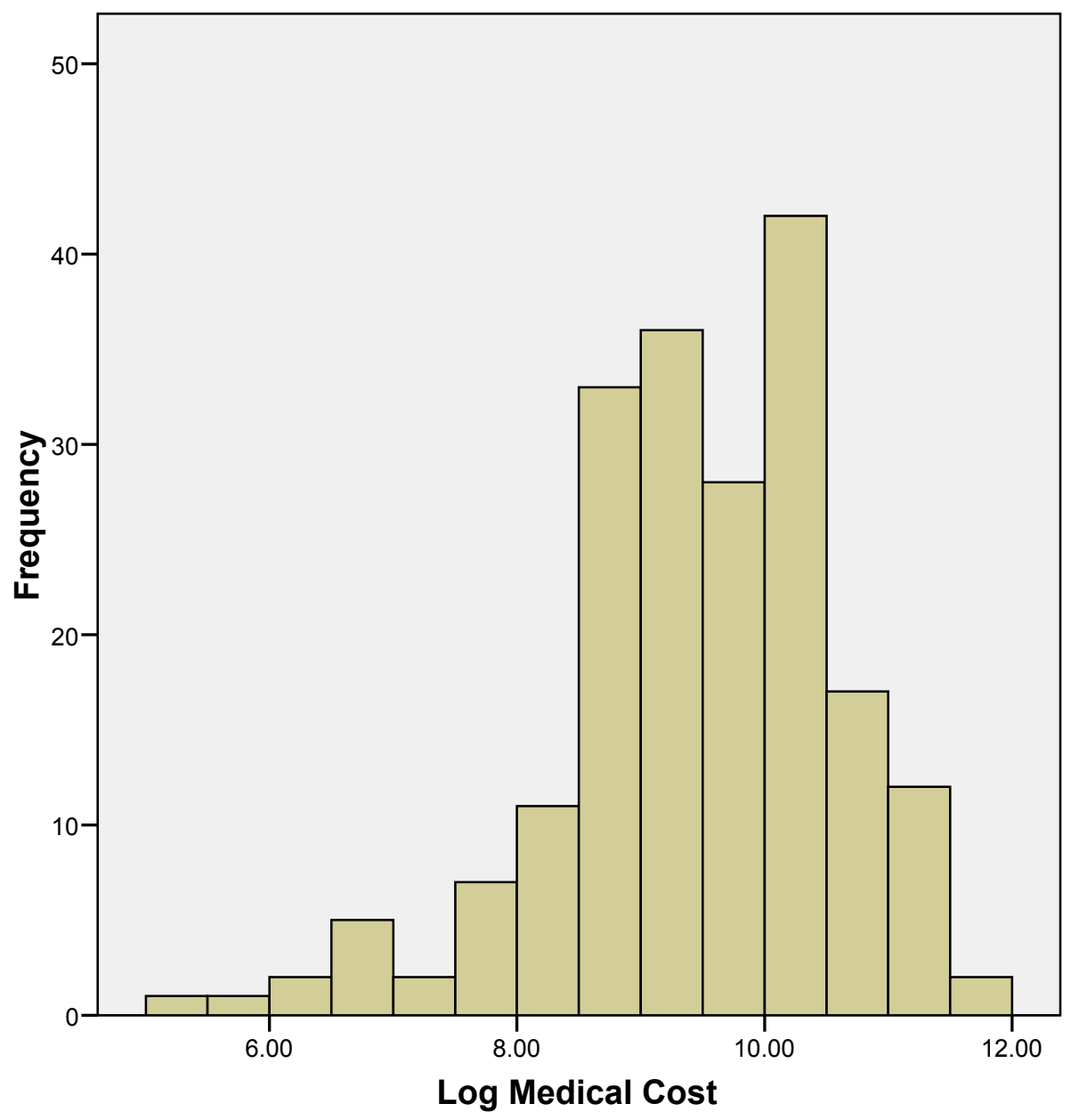

Figure B.7. Histogram of Log Medical Cost for Disability Between $4-8$ months 


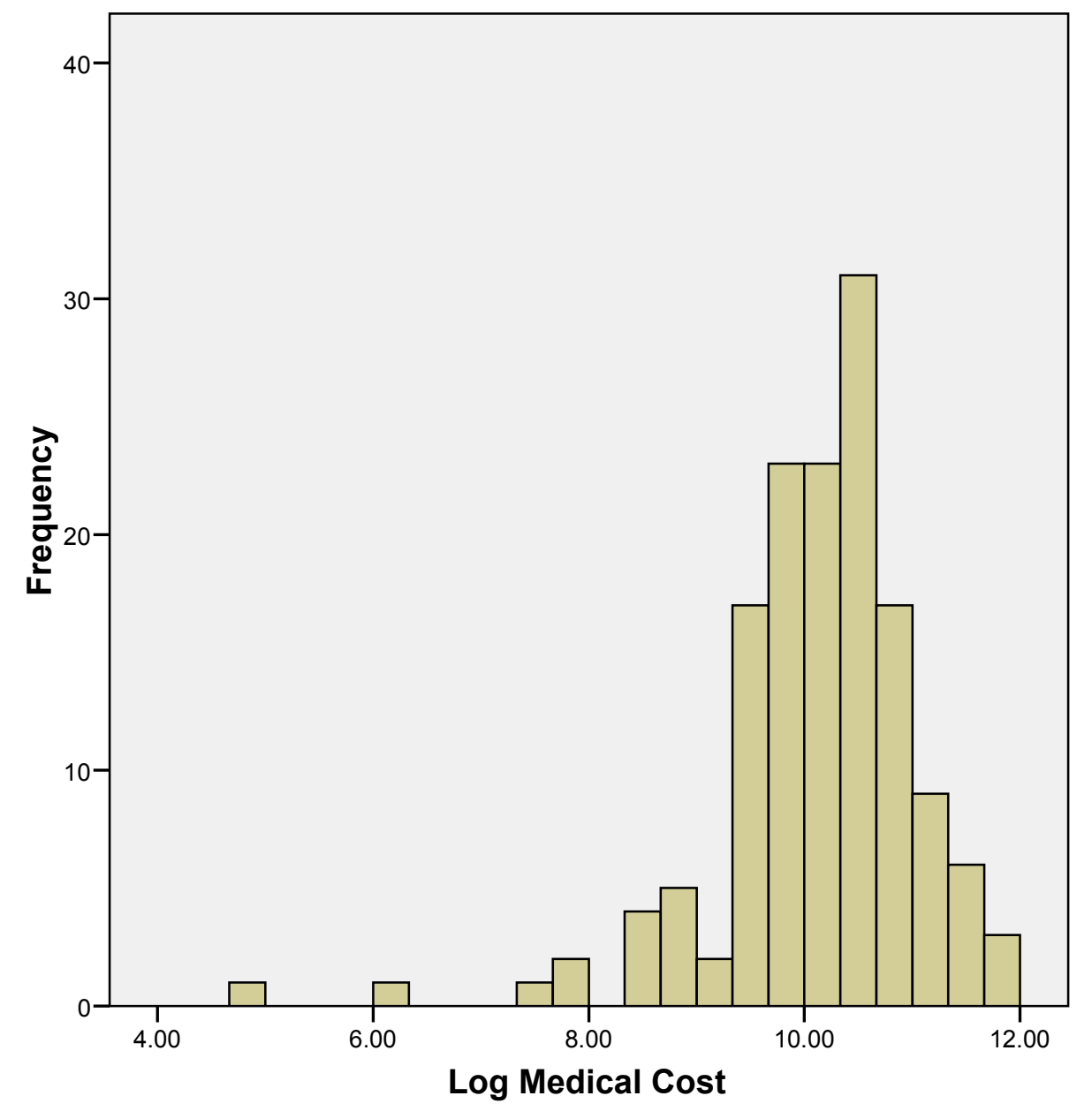

Figure B.8. Histogram of Log Medical Cost for Disability Between 9 - 18 months 


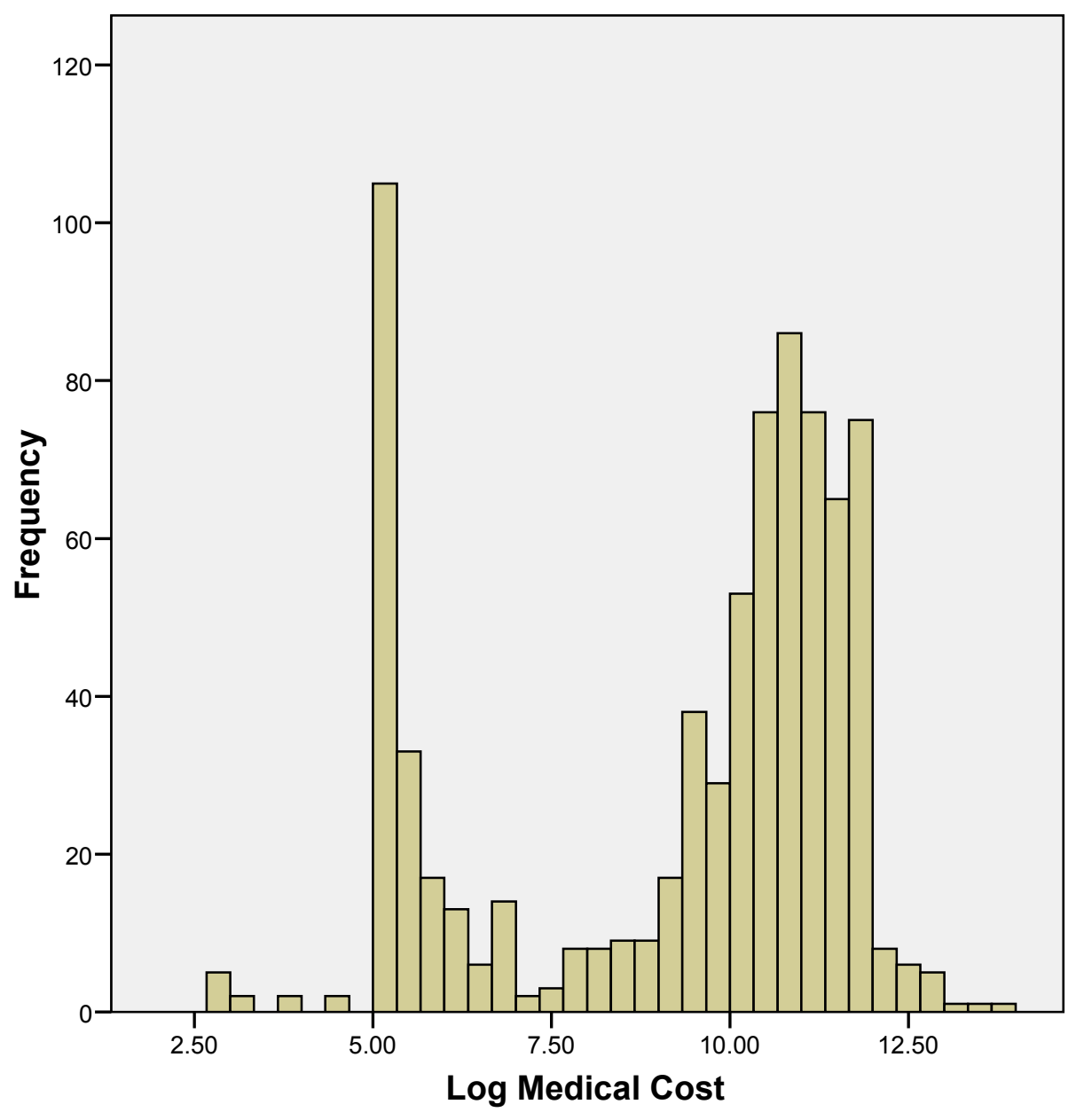

Figure B.9. Histogram of Log Medical Cost for Disability $>18$ months 


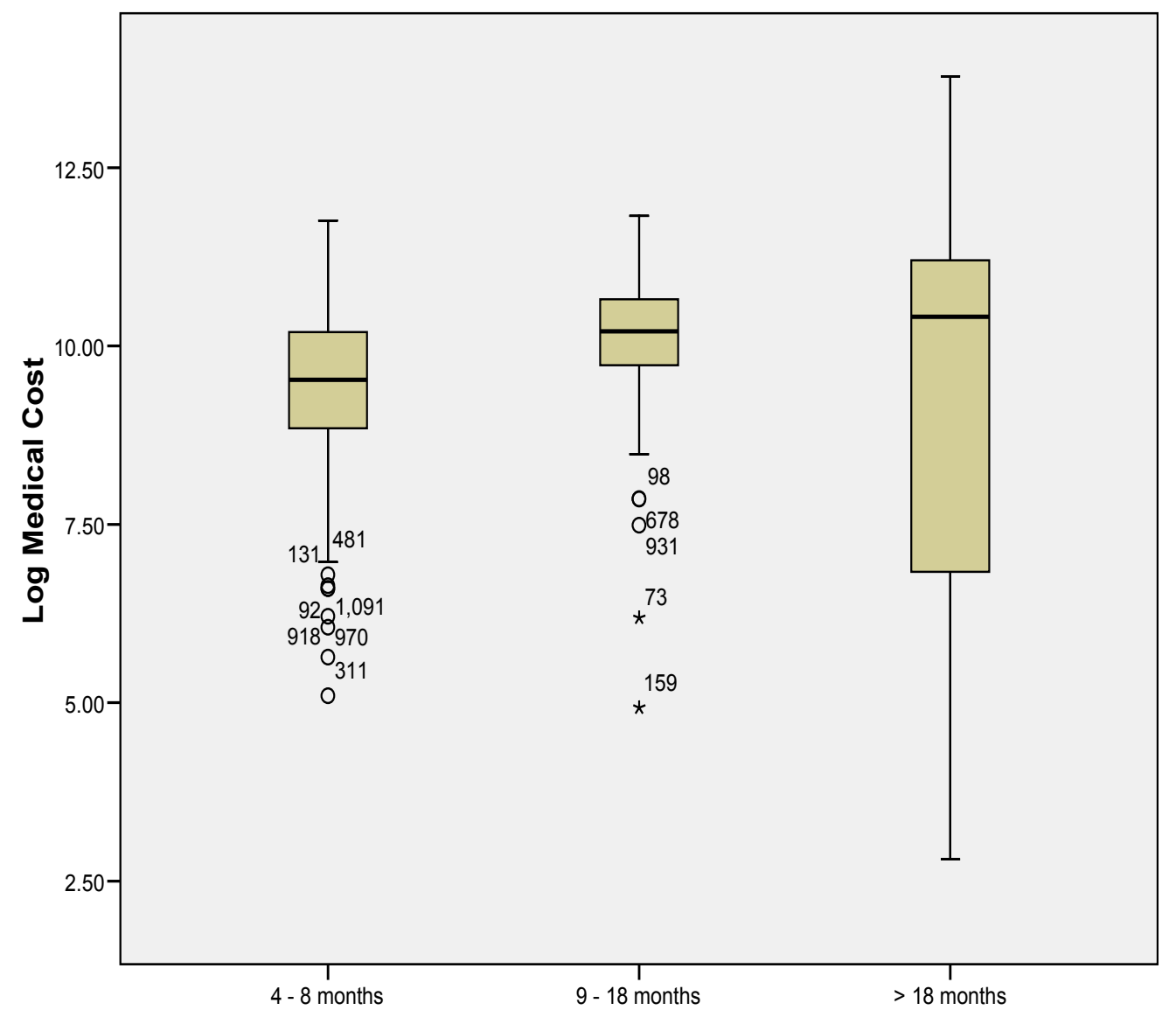

Figure B.10. Boxplots of Disability Duration groups on Re-scaled Medical Cost 
APPENDIX C

COMPUTATION OF TEMPORARY INCOME BENEFITS 


\section{Temporary Income Benefits (TIBs) \\ Information for Injured Employees from the Division of Workers' Compensation}

Income benefits replace a portion of wages you lose because of a work-related injury or illness. There are four types of income benefits:

- temporary income benefits (TIBs);

- impairment income benefits (IIBs);

- supplemental income benefits (SIBs); and

- lifetime income benefits (LIBs).

Income benefits may not exceed the maximum weekly amount set by state law. Temporary income benefits, impairment income benefits, and lifetime income benefits are also subject to a minimum amount set by state law. The maximum and minimum benefit amounts are based on the state average weekly wage.

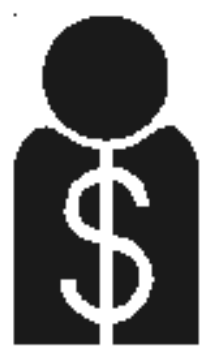

You must report any income (other than workers' compensation benefits you may be receiving) to the Texas Deaprtment of Insurance, Division of Workers' Compensation (TDI-DWC) and the insurance carrier so an adjustment can be made to your income benefit payments. You may be fined and/or charged with fraud if you receive temporary income benefits or supplemental income benefits while also receiving wages from an cmployer without informing the TDI-DWC and the insurance carrier.

Income benefits are not payable following the death of an injured employee. The injured employee's beneficiaries may be eligible to apply and receive death benefits if the injured employee's death was due to the work-related injury or illness.

Temporary Income Benefits (TIBs)

[Tex. Labor Code \$\$408.101 - 408.105, Tex. Admin.

Code 88 s $129.1-129.11]$

You may be paid temporary inoome benefits (TIBs) if your work-related injury or illness causes you to lose all or some of your wages for more than seven (7) days. If you work more than one job, you may be paid TIBs if you lose all or some of your wayes from other employers. (See "Average Weekly Wage Calcualtions" fact sheet under Multiple Employment).

This publication is a summary and is presented for informational purposes only. It is not a substhute for the statute and TDI-DWC rules. For questions about TDI-DWC nules, please call Customer Aesistance at $1-800-252-7031$. CS05-0060 (12-08)

\section{Amount of Temporary Income Benefits}

Temporary income benefits equal 70 percent of the difference between your average weekly wage and the wages you are able to eam after your work-related injury. If you earned less than $\$ 8.50$ par hour before you were injured, your temporary income benefits for the first 26 wedks of payments will equal 75 percent of the difference between your average weekly wage and the wages you are able to eam after your work-related injury.

The amount of temporary income benefits is subject to maximum and minimum benefit amounts. For example, if your average weekly wage was $\$ 500$, and your injury or illness caused you to lose all of your income, your TIBs would be $\$ 350$ a week:

Your average weekly wage

$\$ 500$

Minus your wages after the injury

Lost wages

$\$ 350$

70 percent of $\$ 500(.70 \times 500)$ equals

After an injury, your doctor may release you to retum to work at modified duty; i.e., changes made to your regular job, or a temporary or alternate work assignment. You may still be entitled to TIBs if your employer provides the modified duty at reduced wages.

For example, if your average weekly wage prior to the workrelated injury was $\$ 500$, and you returned to work doing a modified job after the work-related injury and you are now earning \$200 per week working only 4 hours per day, your temporary income benefits would still be $\$ 210$ a week.

Your average weekly wage

$\$ 500$

Minus your wages after the injury

$-\underline{-200}$

Lost wages

$\$ 300$

70 percent of $\$ 300(.70 \times 300)$ equals

$\$ 210$

\author{
For further assistance, call \\ 1-800-252-7031 \\ or visit \\ www.tdi.state.tx.us
}




\section{Temporary Income Benefits (TIBs) Information for Injured Employees from the Division of Workers' Compensation}

Page 2

By retuming to work, you are able to receive a total of $\$ 410$ per week. This includes the wages you are able to earn $(\$ 200)$ plus the TIBs $(\$ 210)$ paid to you by the insurance carrier for lost wages.

\section{When TIBs Begin and End}

You become eligible for TIBs after you miss eight (8) days from work. Remember, disability refers to your inability to eam an income, not to a physical handicap. You have disability if your work-related injury or illness causes you to lose all or some of your usual pay. Benefits are not paid for the first week of lost wages unless disability lasts for two (2) weeks (14 days) or more.

TIBs end at the earlier of.

- the dateyou reach maximum medical improvement (the point that your work-related injury or illness has improved as much as it is going to improve);

- the date you are again physically able to earn your average wedkly wage which would be the same wages you were earning prior to being injured onthe-job; or

- at the end of 104 weeks.

\section{Definitions}

Average Weekly Wage (AWW) is the average amount of weekly wages you earned during the 13 weeks immediately before your work-related injury or illness occurred. Income and death benefit payments are based on your average weekly wage.

Disability occurs when a work-related injury or illness causes you to lose the ability to earn your weekly wages. Disability refers to your ability to eam an income, not to a physical handicap.

Maximum Medical Improvement (MMI) is the earlier of:

- the point in time when your work-related injury or illness has improved as much as it is going to improve or

- 104 weeks from the date you became eligible to receive income benefits.
Maximum Weekly Income Benefit may not exceed 100 percent of the state average weekly wage rounded to the nearest whole dollar. The TDI-DWC will compute the maximum weekly income benefit for Octoba 1 through September 30 of each year no later than October 1 st of each year.

Minimum Weekly Income Benefit is 15 percent of the state average weekly wage rounded to the nearest whole dollar. The TDI-DWC will compute the minimum weekly income benefit for October 1 through September 30 of each year no later than October $1^{\text {tt }}$ of each year.

For more information on Workers' Compensation Benefits see the following fact sheets:

- Workers' Compensation In Texas

- Travel Reimbursement

- Workers' Compensation Benefits

- Dispute Resolution

- Benefit Review Conference

- Contested Case Hearing

- Appeals Rights and Procedures Notice Sent to Parties

- Judicial Review

- Average Wedkly Wage Cal culation 
APPENDIX D

COMPUTATION OF IMPAIRMENT INCOME BENEFITS 


\section{Impairment Income Benefits (IIBs)}

Inceme benefits replace a portion of wages you lose because of a work-related injury or illness. There are four types of income benefits:

- temporary income benefits (TIBs);

- impairment income benefits (IIBs);

- supplemental income benefits (SIBs); and

- lifetime income benefits (LIBs).

Income benefits may not exceed the maximum weekly amount set by state law. Temporary income benefits, impairment income benefits, and lifetime income benefits are also subject to a minimum amount set by state law. The maximum and minimum benefit amounts are based on the state average weekly wage.

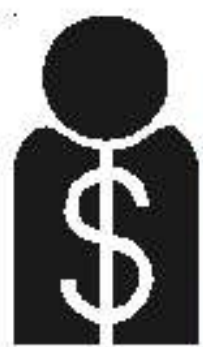

You must report any income (other than income benefits you may be receiving) to the Texas Department of Insurance, Division of Workers' Compensation (TDI-DWC) and the insurance carrier so an adjustment can be made to your income benefit payments. You may be fined and/or charged with fraud if you receive temporary income benefits or supplemental income benefits while also receiving wages from an cmployer without informing the TDI-DWC and the insurance carrier.

Income benefits are not payable following the death of an injured employee. The injured employee's beneficiaries may be eligible to apply and receive death benefits if the injured employee's death was due to the work-related injury or illness.

Impairment Income Benefits (IIBs)

[Tex, Labor Code \$8 808.121 - 408.129; Tex. Admin.

Code s̊sष $130.1-130.11]$

You may be entitled to Impairment Income Benefits (IIBs) if you have a permanent impaiment from a work-related injury or illness. Ganerally, Maximum Medical Improvement (MMI) is reached when you are as well as you are going

This publication is a summary and is presented for informational purposes only. If is not a substhute for the statute and TDI-DWC rules. For questions about TDI-DWC rules, please cal Customer Aesistance at $1-800-252-7031$. CSO5-007E(12-08) to be from the work-related injury or illness. This does not mean that you will not need to follow up care with your health care provider, be completely pain free, or that you are released to return to work. When the health care provider determines you have reached MMI, the health care provider will determine if there is any permanent physical damage. The health care provider will assign an impairment rating (IR) using the $4^{\text {th }}$ Edition of the American Medical Associations (AMA) Guides to the Eval uation of Permanent impairment. The impairment rating describes the degree of permanent damage to your body as a whole.

If you have not previously reached maximum medical improvement, the workers' compensation law establ ishes MMI at 104 weeks. A doctor that is certified by the TDIDWC to do IR examinations must make an assessment of permanent impaimment, if any. If an $\mathbb{R}$ has not been assigned before the 104-week date when your temporary income benefits (TIBs) and, you may not receive IIBs until a doctor assigns an IR. TIBs can no longa be paid after 104 weeks (or maximum medical improvement). Your impairment rating determines whether you are eligible for IIBs. Three (3) weeks of IIBs are paid for each percentage of impairment,

For example, if you receivea 10 percent IR, you will receive 30 wedks of IIBs because 3 weeks of IIBs are paid for each percentage of impairment $(10 \times 3=30$ weeks of IIBs).

\section{Amount of Impairment Income Benefits}

Impairment Income Benefits equal 70 percent of your average weekly wage (AWW). There is a state maximum for impairment income benefits just as there is for TIBs. The maximum for IIBs is 70 percent of the state AWW.

\section{For further assistance, call 1-800-252-7031 or visit www.tdi.state.tx.us}




\section{Impairment Income Benefits (IIBs) \\ Information for Injured Employees from the Division of Workers' Compensation}

Page 2

For example, if your average weekly wage was $\$ 539$, the maximum weokly income benefit would be $\$ 377$.

$\begin{array}{lll}\text { Average weekly wage } & = & \$ 539 \\ 70 \text { percent of } \$ 539 & = & \$ 377\end{array}$

If your average weekly wage was $\$ 500$, your IIB rate would be $\$ 350$.

Average weekly wage $=\$ \$ 500$

70 percent of $\$ 500=\$ 350$

If your average weekly wage is higher than the state average weekly wage (\$750), such as $\$ 836.42$ in the example below, you will not receive 70 percent of $\$ 836.42$, you will receive $\$ 525$ per week, the maximum IIB rate allowed by law.

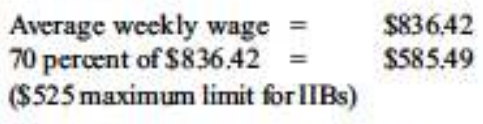

\section{When Impairment Benefits Begin and End}

You become eligible for Impaiment Income Benefits (IIBs) the day after you reach maximum medical improvement (MMI). IIBs end after you have received a total of three (3) weeks of payments for each percentage of your impairment rating.

For example, if you havean impairment rating of 6 percent, you would receive a total of 18 weeks of IIBs. Because IIBs are not wage replacement benefits, you can work while receiving IIBs.

\section{Definitions}

Average Weekly Wage (AWW) is the average amount of weekly wages you earned during the 13 weeks immediately before your work-related injury or illness occurred. Income and death benefit payments are based on your average weekly wage.

Disability occurs when a work related injury or illness causes you to lose the ability to earn your weckly wages. Disability refers to your ability to eam an inoome, not to a physical handicap.
Impairment Rating is the percentage of permanent physical damage to your body that resulted from a workrelated injury or illness.

Maximum Medical Improvement (MMI) is the earlier of:

- the point in time when your work-related injury or illness has improved as much as it is going to improve or

- 104 weeks from the date you became eligible to receive income benefits.

Maximum Weekly Income Benefit may not exceed 100 percent of the state average weekly wage rounded to the nearest whole dollar. The TDI-DWC will compute the maximum weekly income benefit for Octoba 1 through September 30 of each year no later than October $1^{\text {st }}$ of each year.

Minimum Weekly Income Benefit is 15 percent of the state average weekly wage rounded to the nearest whole dollar. The TDI-DWC will compute the maximum weekly income benefit for October 1 through September 30 of each year no later than October $1^{\text {tt }}$ of each year.

\section{For more information on Workers' Compensation} Benefits see the following fact sheets:

- Workers' Compensation In Texas

- Travel Reimbursement

- Workers' Compensation Benefits

- Dispute Resolution

- Benefit Review Conference

- Contested Case Hearing

- Appeals Rights and Procedures Notice Sent to Parties

- Judicial Review 


\section{APPENDIX E}

COMPUTATION OF SUPPLEMENTAL INCOME BENEFITS 


\section{Supplemental Income Benefits (SIBs) Information for Injured Employees from the Division of Workers' Compensation}

Inceme benefits replace a portion of wages you lose because of a work-related injury or illness. There are four types of income benefits:

- temporary income benefits (TIBs);

- impairment income benefits (IIBs);

- supplemental income benefits (SIBs); and

- lifetime income benefits (LIBs).

Income benefits may not exceed the maximum weekly amount set by state law. Temporary income benefits, impairment income benefits, and lifetime income benefits are also subject to a minimum amount. The maximum and minimum benefit amounts are based on the state average weekly wage.

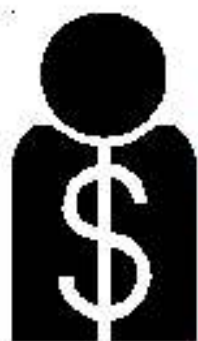

You must report any income (other than workers' compensation benefits you may be receiving) to the Texas Department of the Insurance, Division of Workers' Compensation (TDIDWC) and the insurance carrier so an adjustment can be made to your income benefit payments. You may be fined and/or charged with fraud if you receive temporary income benefits and supplemental income benefits while also receiving wages from an employer without informing the TDI-DWC and the insurance carrier.

Income benefits are no longer payable following the death of an injured employee. The injured employees's beneficiaries may be eligible to apply and receive death benefits if the injured employee's death was due to the work-related injury or illness.

Supplemental Inceme Benefits (SIBs)

[Sections 408.141-408.151, Rules 130.100-130.108]

Supplement Income Benefits (SIBs) are income benefits that you apply for quarterly (4 times per year, or every 3 months) and are paid monthly to injured employees that meet specific eligibility requirements.

This publication is a summary and is presented for informational purposes only. It is not a substzute for the statute and TDI-DWC rules. For questions about TDI-DWC rules, please cal Customer Aesistance at $1-800-252-7031$. CSO5-008D(3-08)
You may be entitled to SIBs if you meet the following entitlement requirements:

- you have an impaimnent rating of 15 percent or more;

- you have not returned to work because of your impairment, or you have returned to work but are earning less than 80 percent of the average weekly wage because of the impaiment;

- you have made a good faith effort to find a job that matches your abilities to work, and

- you did not receive your impairment income benefits in a lump sum payment.

\section{Amount of Supplemental Income Benefits}

Supplemental Income Benefits equal 80 percent of the difference between 80 percent of your average weekty wage (earned prior to your work-related injury) and your wedkly wages (if you have any eamings or offered wages during this 13 week period) after the work-related injury.

For example, if your average weekly wage was $\$ 500$ before you were injured, and your injury caused you to lose all of your income, your $\mathrm{SIB}$ rate would be $\$ 320$ a week:

Your average weekly wage

$\$ \$ 00$

80 percent of $\$ 500(.80 \times 500)$ equals

Minus your wage now

Equals

80 percent of $\$ 400(.80 \times 400)$ equals

$\$ 320$

Supplemental income benefits are paid monthly. To determine the amount of your monthly SIBs, multiply the wedkly benefit amount by the average number of weeks in a month (4.34821). In this example, your monthly supplemental income benefit would be $\$ 1,391,43$ :

$\$ 320 \times 4.34821$ equals $\$ 1,391.43$

\author{
For further assistance, call \\ 1-800-252-7031 \\ or visit \\ www.tdi.state.tx.us
}




\section{Supplemental Income Benefits (SIBs) Information for Injured Employees from the Division of Workers' Compensation}

Page 2

If you eam any wages during the qualifying period, the wages are deducted when calculating your SIB rate.

\author{
Example: \\ Your average weekly wage $\quad \$ 500$ \\ 80 percent of $\$ 500(.80 \times 500)$ equals $\quad 400$ \\ Minus your wages eamed 200 \\ Equals \\ 200 \\ 80 percent of $\$ 200(.80 \times 200)$ equals $\$ 160$ \\ $\$ 160 \times 4.34821$ equals $\$ 695.71$ (monthly SIB rate)
}

When Supplemental Benefits Begin and End

If you are eligible, Supplemental Income Benefits will begin the day after your Impairment Income Benefits end. The TDI-DWC will automatically notify you if your impaiment rating is 15 percent or greater and inform you of what information is necessary to support your application for the 1 st quarter of SIBs. The application for the 1 st quarter of SIBs is sent to the TDI-DWC. The information to support your entitlement to the first quarter of SIBs is based on your job search efforts (if able) and wages carned during the 13 weeks before the end of your IIBs period. This $13-$ week period is called the qualifying period.

The TDI-DWC will make a determination of entitlement based on the information on your application. The TDIDWC will review your job search efforts during the qualifying period, any possible job offers, current medical documentation provided by your doctor supporting why you are unableto work (if applicable), and will look at whether your inability to earn your pro-injury wage is a direct result of your impairment.

The insurance carrier will provide you with an application for future quarters of SIBs with the first payment of any quarter or with any notice of non-entitl ament. You must send all other SIB applications to the insurance carrier showing that you are eligible to receive SIBs. If the insurance carrier agrees you are eligible, you will receive benefits for the quarter. Each time you file an application with the insurance carrier, you must show that:

- you looked for work each week of the qualifying period; or

- you have current medical documentation from your doctor that explains clearly why your work-related injury or illness prevented you from working or looking for work during that 13 week timeframe; and

- your cooperation with the Texas Department of Assistive \& Rehabilitative Services, or a private provider of vocational rehabilitation.

Your entitlement to receive supplemental income benefits ends at 401 weeks (approximately $71 / 2$ years) from the date of your injury. If you have an occupational illness, entitlement for supplement income benefits ands at 401 weoks from the date you first became eligible to receive income benefits. If you are not entiteled to SIBs for four consecutive quarters (one year), you permanently lose entitlement to those benefits.

\section{Definitions}

Average Weekly Wage (AWW) is the average amount of weekly wages you earned during the 13 weeks immediately before your work-related injury or illness occurred. Income and death benefit payments are based on your avenage weekly wage.

Disability occurs when a work-related injury or illness causes you to lose the ability to earn your weekly wages. Disability refers to your ability to eam an income, not to a physical handicap.

Impairment Rating is the percentage of permanent physical damage to your body that resulted from a workrelated injury or illness.

Maximu m Benefit Amount may not exceed 100 pervent of the state average weekly wage rounded to the nearest whole dollar, The TDI-DWC will compute the maximum weekly income benefit for each state fiscal year no later than September $1^{*}$ of each year.

Minimum Benefit Amount is 15 percent of the state average weekly wage rounded to the nearest whole dollar. The TDI-DWC will compute the minimum weekly income benefit for each state fiscal year no later than September $1^{\text {tt }}$ of each year. 
APPENDIX F

DATA FROM THE EUGENE MCDERMOTT CENTER FOR PAIN MANAGEMENT 
Appendix F. One-Year SF-36 Scores and Corresponding Quality of Life Score from the Interdisciplinary Pain Management Program at the Eugene McDermott Center for Pain Management $(\mathrm{N}=93)$.

\begin{tabular}{|l|ccc|}
\hline \multirow{2}{*}{$\begin{array}{l}\text { Quality of Life } \\
\text { Measures at One-Year* }\end{array}$} & \multicolumn{3}{|c|}{ McDermott Disability Groups } \\
& $4-8$ months & $9-18$ months & $>18$ months \\
& $\mathrm{N}=18$ & $\mathrm{~N}=17$ & $\mathrm{~N}=58$ \\
\hline SF- 36 (SD) & & & \\
Mental Component & $47.9(14.0)$ & $46.9(13.9)$ & $46.4(13.1)$ \\
Physical Component & $36.2(9.2)$ & $33.9(8.0)$ & $32.1(10.1)$ \\
Physical Functioning & $52.3(28.5)$ & $52.2(25.6)$ & $47.6(27.5)$ \\
Role Physical & $41.7(33.2)$ & $36.8(40.6)$ & $29.8(42.4)$ \\
Bodily Pain & $45.8(20.0)$ & $42.1(20.6)$ & $39.8(21.4)$ \\
General Health & $61.5(25.9)$ & $50.3(23.5)$ & $49.3(26.1)$ \\
Vitality & $43.8(25.8)$ & $44.6(27.1)$ & $39.7(24.5)$ \\
Social Functioning & $64.9(27.7)$ & $55.1(33.1)$ & $53.4(29.9)$ \\
Role Emotional & $66.7(42.8)$ & $68.6(44.8)$ & $63.7(46.4)$ \\
Mental Health & $68.7(28.8)$ & $63.4(27.6)$ & $65.5(22.9)$ \\
& & & $0.61(0.07)$ \\
\hline One-Year Quality & $0.63(0.06)$ & $0.63(0.05)$ & \\
of Life Scores (SD) & & & \\
& & & \\
\hline
\end{tabular}

* No significant differences among the three disability duration groups on all measures. 


\section{REFERENCES}

Aaron, L. A., Burke, M. M., \& Buchwald, D. (2000). Overlapping conditions among patients with chronic fatigue syndrome, fibromyalgia, and temporomandibular disorder. Archives of Internal Medicine, 160(2), 221-227.

American Medical Association. (2008). Guides to the Evaluation of Permanent

Impairment (6th ed.). Chicago, IL: American Medical Association.

American Psychiatric Association. (2000). Diagnostic and Statistical Manual of Mental Disorders, 4th Edition, Text Revision. Washington, D. C.: American Psychiatric Association.

Anagnostis, C., Mayer, T. G., Gatchel, R. J., \& Proctor, T. (2003). The Million Visual Analog Scale: Its utility for predicting tertiary rehabilitation outcomes. Spine, 28, 1-10.

Arnow, B. A., Hunkeler, E. M., Blasey, C. M., Lee, J., Constantino, M. J., Fireman, B., et al. (2006). Comorbid depression, chronic pain, and disability in primary care. Psychosom Med, 68(2), 262-268.

Aronoff, G. M., Mandel, S., Genovese, E., Maitz, E. A., Dorto, A. J., Klimek, E. H., et al. (2007). Evaluating malingering in contested injury or illness. Pain Pract, $7(2), 178-204$.

Arrow, K. J. (1963). Uncertainty and the welfare economics of medical care. American Economic Review, 53, 941 - 973. 
Aschengrau, A., \& Seage, G. R. (2003). Essentials of Epidemiology in Public Health. London: Jones \& Bartlett Publishers.

Becker, N., Sjogren, P., Beck, P., Olsen, A. K., \& Eriksen, J. (2000). Treatment outcome of chronic non-malignant pain patients managed in a Danish multidisciplinary pain centre compared to general practice: A randomised controlled trial. Pain, 84(2-3), 203-211.

Bendix, A. E., Bendix, T., Vaegter, K., Lund, C., Frolund, L., \& Holm, L. (1996). Multidisciplinary intensive treatment for chronic low back pain: A randomized, prospective study. Cleveland Clinic Journal of Medicine, 63(1), 62-69.

Bhandari, M., Busse, J. W., Hanson, B. P., Leece, P., Ayeni, O. R., \& Schemitsch, E. H. (2008). Psychological distress and quality of life after orthopedic trauma: an observational study. Can J Surg, 51(1), 15-22.

Brazier, J., Roberts, J., \& Deverill, M. (2002). The estimation of a preference-based measure of health from the SF-36. J Health Econ, 21(2), 271-292.

Brazier, J. E., \& Roberts, J. (2004). The estimation of a preference-based measure of health from the SF-12. Med Care, 42(9), 851-859.

Chernick, M. R. (1999). Bootstrap Methods: A practitioner's guide. New York: John Wiley \& Sons.

Chibnall, J. T., Tait, R. C., Andresen, E. M., \& Hadler, N. M. (2006). Clinical and social predictors of application for social security disability insurance by workers' compensation claimants with low back pain. J Occup Environ Med, 48(7), 733-740. 
Corey, D. T., Koepfler, L. E., Etlin, D., \& Day, H. I. (1996). A limited functional restoration program for injured workers: A randomized trial. Journal of Occupational Rehabilitation, 6, 239-249.

Critchley, D. J., Ratcliffe, J., Noonan, S., Jones, R. H., \& Hurley, M. V. (2007). Effectiveness and cost-effectiveness of three types of physiotherapy used to reduce chronic low back pain disability: a pragmatic randomized trial with economic evaluation. Spine, 32(14), 1474-1481.

Culyer, A. J. (1989). The normative economics of health care finance and provision. Oxford Review of Economic Policy, 5(1), 34 - 58.

Dasbach, E. J., \& Teutsch, S. M. (2003). Quality of Life. In A. C. Haddix, S. M. Teutsch \& P. S. Corso (Eds.), Prevention effectiveness: A guide to decision analysis and economic evaluation (2nd ed., pp. 77-91): Oxford University Press.

DeBerard, M. S., Masters, K. S., Colledge, A. L., Schleusener, R. L., \& Schlegel, J. D. (2001). Outcomes of posterolateral lumbar fusion in Utah patients receiving workers' compensation: a retrospective cohort study. Spine, 26(7), 738-746; discussion 747 .

Dersh, J., Gatchel, R. J., Mayer, T., Polatin, P., \& Temple, O. (2006). Prevalence of psychiatric disorders in patients with chronic disabling occupational spinal disorders. Spine, 31, 1156-1182.

Dersh, J., Mayer, T., Theodore, B. R., Polatin, P., \& Gatchel, R. J. (2007). Do psychiatric disorders first appear preinjury or postinjury in chronic disability occupational spinal disorders? Spine, 32(9), 1045-1051. 
Dersh, J., Mayer, T. G., Gatchel, R. J., Polatin, P. B., Theodore, B. R., \& Mayer, E. A. (2008). Prescription opioid dependence is associated with poorer outcomes in disabling spinal disorders. Spine, 33(20), 2219-2227.

Dersh, J., Mayer, T. G., Gatchel, R. J., Towns, B., Theodore, B. R., \& Polatin, P. (2007). Psychiatric comorbidity in chronic disabling occupational spinal disorders has minimal impact on functional restoration socioeconomic outcomes. Spine, 32(17), 1917-1925.

Dersh, J., Polatin, P., \& Gatchel, R. (2002). Chronic pain and psychopathology: Research findings and theoretical considerations. Psychosomatic Medicine, 64, $773-786$.

Dersh, J., Polatin, P. B., Leeman, G., \& Gatchel, R. J. (2004). The Management of Secondary Gain and Loss in Medicolegal Settings: Strengths and Weaknesses. Journal of Occupational Rehabilitation, 14(4), 267-279.

Deschner, M., \& Polatin, P. B. (2000). Interdisciplinary programs: Chronic pain management. In T. G. Mayer, R. J. Gatchel \& P. B. Polatin (Eds.), Occupational Musculoskeletal Disorders: Function, Outcomes \& Evidence (pp. 629-637). Philadelphia: Lippincott, Williams \& Wilkins.

Dicker, R. C. (2002). Designing studies in the field. In M. B. Gregg (Ed.), Field Epidemiology (2nd ed.). New York, NY: Oxford University Press.

Drummond, M. F., O'Brien, B., Stoddart, G. L., \& Torrance, G. W. (1997). Methods for the economic evaluation of health care programmes. Oxford: Oxford University Press. 
Eccleston, S. M., \& Zhao, X. (2005). The Anatomy of Workers' Compensation Medical Costs and Utilization in Texas, (5th ed.). Cambridge, MA: Workers' Compensation Research Institute.

Engel, G. L. (1977). The need for a new medical model: A challenge for biomedicine. Science, 196(4286), 129-136.

Epstein, S. A., Kay, G., Clauw, D., Heaton, R., Klem, D., Krupp, L., et al. (1999). Psychosomatic disorders in patients with fibromyalgia: A multicenter investigation. Psychosomatics, 40, 57-63.

Farnham, P. G., \& Haddix, A. C. (2003). Study Design. In A. C. Haddix, S. M. Teutsch \& P. S. Corso (Eds.), Prevention effectiveness: A guide to decision analysis and economic evaluation (2nd ed.). New York: Oxford University Press.

Feeny, D., Furlong, W., Torrance, G. W., Goldsmith, C. H., Zhu, Z., DePauw, S., et al. (2002). Multiattribute and single-attribute utility functions for the health utilities index mark 3 system. Med Care, 40(2), 113-128.

Fenwick, E., Claxton, K., \& Sculpher, M. (2001). Representing uncertainty: the role of cost-effectiveness acceptability curves. Health Econ, 10(8), 779-787.

Fernandez, E. (1998). The role of affect in somatoform and factitious disorders. Current Review of Pain, 2, 109-114.

First, M., Spitzer, R., Gibbon, M., \& Williams, J. (1997a). Structured Clinical Interview for DSM-IV Axis I Disorders, Research Version, Non-patient Edition (SCIDI/NP). New York: Biometrics Research, New York State Psychiatric Institute. 
First, M., Spitzer, R., Gibbon, M., \& Williams, J. (1997b). Structured Clinical Interview for DSM-IV Personality Disorders (SCID-II). Washington, D.C.: American Psychiatric Press, Inc.

Fishbain, D. A. (1994). Secondary gain concept: Definition problems and its abuse in medical practice. APS Journal, 3(4), 264-273.

Flor, H., \& Turk, D. (1988). Chronic back pain and rheumatoid arthritis: Predicting pain and disability from cognitive variables. Journal of Behavioral Medicine, $11(3), 251-265$.

Flynn, J. C., \& Hoque, M. A. (1979). Anterior fusion of the lumbar spine. End-result study with long-term follow-up. J Bone Joint Surg Am, 61(8), 1143-1150.

Folland, S., Goodman, A. C., \& Stano, M. (2001). The economics of health and health care (3rd ed.). Upper Saddle River, NJ: Prentice Hall.

Franche, R., Frank, J., \& Krause, N. (2005). Prediction of occupational disability: Models, factors and outcomes. In I. Schultz \& R. Gatchel (Eds.), Handbook of complex occupational disability claims: Early risk identification, intervention, and prevention. (pp. 93-116). New York: Springer.

Franche, R. L., \& Krause, N. (2003). Critical factors in recovery and return to work. In T. Sullivan \& J. Frank (Eds.), Preventing and Managing Disabling Injury at Work (pp. 33-57). New York: Taylor \& Francis.

Franklin, G., Haug, J., Heyer, N., McKeefrey, S., \& Picciano, J. (1994). Outcome of lumbar fusion in Washington state worker's compensation. Spine, 17, 18971904. 
Franklin, G. M., Mai, J., Wickizer, T., Turner, J. A., Fulton-Kehoe, D., \& Grant, L. (2005). Opioid dosing trends and mortality in Washington State workers' compensation, 1996-2002. American Journal of Industrial Medicine, 48(2), 9199.

Franks, P., Lubetkin, E. I., Gold, M. R., \& Tancredi, D. J. (2003). Mapping the SF-12 to preference-based instruments: convergent validity in a low-income, minority population. Med Care, 41(11), 1277-1283.

Franks, P., Lubetkin, E. I., Gold, M. R., Tancredi, D. J., \& Jia, H. (2004). Mapping the SF-12 to the EuroQol EQ-5D Index in a national US sample. Med Decis Making, 24(3), 247-254.

Fryback, D. G., \& Lawrence, W. F., Jr. (1997). Dollars may not buy as many QALYs as we think: a problem with defining quality-of-life adjustments. Med Decis Making, 17(3), 276-284.

Fryback, D. G., Lawrence, W. F., Martin, P. A., Klein, R., \& Klein, B. E. (1997). Predicting Quality of Well-being scores from the SF-36: results from the Beaver Dam Health Outcomes Study. Med Decis Making, 17(1), 1-9.

Frymoyer, J. W., \& Durett, C. L. (1997). The economics of spinal disorders. In J. W. Frymoyer, T. B. Ducker, J. P. Kostuik, J. N. Weinstein \& T. S. I. Whitecloud (Eds.), The Adult Spine: Principles and Practice (2nd ed., Vol. 1, pp. 143-150). Philadelphia: Lippincott-Raven.

Garber, A. M., Weinstein, M. C., Torrance, G. W., \& Kamlet, M. S. (1996). Theoretical foundations of cost-effectiveness analysis. In R. M. Gold, J. E. Siegel, L. B. 
Russell \& M. C. Wienstein (Eds.), Cost-effectiveness in health and medicine. New York: Oxford University Press.

Gasper, D. (1997). Sen's capability approach and Nussbaum's capabilities ethics. Journal of International Development, 9(2), 281 - 302.

Gatchel, R. J. (1991). Early development of physical and mental deconditioning in painful spinal disorders. In T. G. Mayer, V. Mooney \& R. J. Gatchel (Eds.), Contemporary Conservative Care for Painful Spinal Disorders (pp. 278-289). Philadelphia: Lea \& Febiger.

Gatchel, R. J. (1996). Psychological disorders and chronic pain: Cause and effect relationships. In R. J. Gatchel \& D. C. Turk (Eds.), Psychological Approaches to Pain Management: A Practitioner's Handbook (pp. 33-52). New York: Guilford.

Gatchel, R. J. (2002). Treatment of Patients with Temporomandibular Disorders. In D. C. Turk \& R. J. Gatchel (Eds.), Psychological Approaches to Pain Management: A Practitioner's Handbook (2nd. ed.). New York: Guilford.

Gatchel, R. J. (2004). Comorbidity of chronic mental and physical health disorders: The biopsychosocial perspective. American Psychologist, 59, 792-805.

Gatchel, R. J., \& Mayer, T. G. (2000). Occupational Musculoskeletal Disorders: Introduction and Overview of the Problem. In T. G. Mayer, R. J. Gatchel \& P. B. Polatin (Eds.), Occupational Musculoskeletal Disorders: Function, Outcomes, and Evidence (pp. 3-8). Philadelphia: Lippincott Williams \& Wilkins. 
Gatchel, R. J., Mayer, T. G., Dersh, J., Robinson, R., \& Polatin, P. B. (1999). The association of the SF-36 Health Status Survey with one-year socioeconomic outcomes in a chronically disabled spinal disorder population. Spine, 24, 21622170 .

Gatchel, R. J., Mayer, T. G., Kidner, C. L., \& McGeary, D. D. (2005). Are gender, marital status or parenthood risk factors for outcome of treatment for chronic disabling spinal disorders? J Occup Rehabil, 15(2), 191-201.

Gatchel, R. J., Mayer, T. G., \& Theodore, B. (2006). The Pain Disability Questionnaire: Relationship to one-year functional and psychosocial rehabilitation outcomes. Journal of Occupational Rehabilitation, 16(1), 75-94.

Gatchel, R. J., \& Okifuji, A. (2006). Evidence-based scientific data documenting the treatment and cost-effectiveness of comprehensive pain programs for chronic nonmalignant pain. J Pain, 7(11), 779-793.

Gatchel, R. J., \& Oordt, M. S. (2003). Clinical Health Psychology and Primary Care: Practical Advice and Clinical Guidance for Successful Collaboration. Washington, DC: American Psychological Association.

Gatchel, R. J., Polatin, P. B., Noe, C., Gardea, M., Pulliam, C., \& Thompson, J. (2003). Treatment- and cost-effectiveness of early intervention for acute low-back pain patients: a one-year prospective study. J Occup Rehabil, 13(1), 1-9.

Gatchel, R. J., Stowell, A. W., Wildenstein, L., Riggs, R., \& Ellis, E. (2006). Efficacy of an early intervention for patients with acute temporomandibular disorder- 
related pain: A one-year outcome study. Journal of the American Dental Association, 137, 339-347.

Gatchel, R. J., \& Turk, D. C. (1996). Psychological Approaches to Pain Management: A Practitioner's Handbook. New York: Guilford Publications, Inc.

Gift, T. L., Haddix, A. C., \& Corso, P. S. (2003). Cost-Effectiveness Analysis. In A. C. Haddix, S. M. Teutsch \& P. S. Corso (Eds.), Prevention effectiveness: A guide to decision analysis and economic evaluation (2nd ed.). New York: Oxford University Press.

Gold, M. R., Patrick, D. L., Torrance, G. W., Fryback, D. G., Hadorn, D. C., Kamlet, M. S., et al. (1996). Identifying and valuing outcomes. In M. R. Gold, J. E. Siegel, L. B. Russell \& M. C. Weinstein (Eds.), Cost-effectiveness in health and medicine. New York: Oxford University Press.

Gold, M. R., Siegel, J. E., Russell, L. B., \& Weinstein, M. C. (Eds.). (1996). Costeffectiveness in health and medicine. New York: Oxford University Press.

Goldie, S. J., \& Corso, P. S. (2003). Decision Analysis. In A. C. Haddix, S. M. Teutsch \& P. S. Corso (Eds.), Prevention effectiveness: A guide to decision analysis and economic evaluation (2nd ed.). New York: Oxford University Press.

Gracely, R. H., Petzke, F., Wolf, J. M., \& Clauw, D. J. (2002). Functional magnetic resonance imaging evidence of augmented pain processing in fibromyalgia. Arthritis and Rheumatism, 46, 1333-1343.

Greene, C. S. (2001). The etiology of temporomandibular disorders: Implications for treatment. Journal of Orofacial Pain, 15(2), 90-105. 
Gremillion, H. A. (2000). The Prevalence and Etiology of Temporomandibular Disorders and Orofacial Pain. Texas Dental Journal(July), 30-39.

Guzman, J., Esmail, R., Karjalinen, K., Malmivaara, A., Irvin, E., \& Bombadier, C. (2001). Multidisciplinary rehabilitation for chronic low back pain: Systematic review. British Medical Journal, 322, 1511-1516.

Gyrd-Hansen, D. (2005). Willingness to pay for a QALY: theoretical and methodological issues. Pharmacoeconomics, 23(5), 423-432.

Haddix, A. C., Corso, P. S., \& Gorsky, R. D. (2003). Costs. In A. C. Haddix, S. M. Teutsch \& P. S. Corso (Eds.), Prevention effectiveness: A guide to decision analysis and economic evaluation (2nd ed., pp. 53-76). New York: Oxford University Press.

Haddix, A. C., Teutsch, S. M., \& Corso, P. S. (Eds.). (2003). Prevention effectiveness: A guide to decision analysis and economic evaluation (2nd ed.). New York: Oxford University Press.

Hagen, E. M., Eriksen, H. R., \& Ursin, H. (2000). Does early intervention with a light mobilization program reduce long-term sick leave for low back pain? Spine, 25(15), 1973-1976.

Hamilton, M. (1960). A rating scale for depression. Journal of Neurology, Neurosurgery \& Psychiatry, 23, 56-62.

Hashemi, L., Webster, B., \& Clancy, E. (1998). Trends in disability duration and cost of workers' compensation low back pain claims (1988-1996). JOEM, 40(12), 11101119. 
Hashemi, L., Webster, B., Clancy, E., \& Volinn, E. (1997). Length of disability and cost of workers' compensation low back pain claims. Journal of Occupational Rehabilitation, 39(10), 937-945.

Hashemi, L., Webster, B. S., Clancy, E. A., \& Courtney, T. K. (1998). Length of disability and cost of work-related musculoskeletal disorders of the upper extremity. J Occup Environ Med, 40(3), 261-269.

Hatten, A. L., Gatchel, R. J., Polatin, P. B., \& Stowell, A. W. (2006). A cost-utility analysis of chronic spinal pain treatment outcomes: converting SF-36 data into quality-adjusted life years. Clin J Pain, 22(8), 700-711.

Hays, W. L. (1994). Statistics (5 ed.). Fort Worth: Harcourt Brace.

Hazard, R. G. (1995). Spine update: Functional restoration. Spine, 20, 2345-2348.

Hazard, R. G., Fenwick, J. W., Kalisch, S. M., Redmond, J., Reeves, V., Reid, S., et al. (1989). Functional restoration with behavioral support: A one-year prospective study of patients with chronic low-back pain. Spine, 14, 157-161.

Heitjan, D. F. (2008). ICER vs. INB: The Statistical Issues. Paper presented the International Conference on Health Policy Statistics, Philadelhpia, PA. January 17th.

Heitjan, D. F., Moskowitz, A. J., \& Whang, W. (1999). Problems with interval estimates of the incremental cost-effectiveness ratio. Med Decis Making, 19(1), 9-15. 
Hildebrandt, J., Pfingsten, M., Saur, P., \& Jansen, J. (1997). Prediction of success from a multidisciplinary treatment program for chronic low back pain. Spine, 22, 9901001.

Hodgson, T. A. (1994). Cost of illness in cost-effectiveness analysis: a review of the methodology. Pharmacoeconomics, 6, 536-552.

Hollingworth, W., Deyo, R. A., Sullivan, S. D., Emerson, S. S., Gray, D. T., \& Jarvik, J. G. (2002). The practicality and validity of directly elicited and SF-36 derived health state preferences in patients with low back pain. Health Econ, 11(1), 7185.

Howard, K. J., Mayer, T. G., Theodore, B. R., Shea, M., \& Gatchel, R. J. (in press). Chronic disabling occupational musculoskeletal disorder patients failing to complete functional restoration: Analysis of treatment-resistant personality characteristics. Archives of Physical Medicine and Rehabilitation.

Indahl, A., Haldorsen, E. H., Holm, S., Reikers, O., \& Holger, U. (1998). Five-year follow-up study of a controlled clinical trial using light mobilization and an informative approach to low back pain. Spine, 23, 2625-2630.

Ives, T. J., Chelminski, P. R., Hammett-Stabler, C. A., Malone, R. M., Perhac, J. S., Potisek, N. M., et al. (2006). Predictors of opioid misuse in patients with chronic pain: a prospective cohort study. BMC Health Serv Res, 6, 46.

Johannesson, M. (1995). The relationship between cost-effectiveness analysis and costbenefit analysis. Soc Sci Med, 41(4), 483-489. 
Jordan, K. D., Mayer, T. G., \& Gatchel, R. J. (1998). Should extended disability be an exclusion criterion for tertiary rehabilitation? Socioeconomic outcomes of early versus late functional restoration in compensation spinal disorders. Spine, 23(19), 2110-2116; discussion 2117.

Jousset, N., Fanello, S., Bontoux, L., Dubus, V., Billabert, C., Vielle, B., et al. (2004). Effects of functional restoration versus 3 hours per week physical therapy: A randomized controlled study. Spine, 29(5), 487-493.

Kahneman, D., \& Tversky, A. (1979). Prospect Theory: An analysis of decision under risk. Econometrica, 47, 263 - 291.

Kaplan, R. M., \& Anderson, J. P. (1993). The Quality of Well-being Scale: Rationale for a single quality of life index. In S. R. Walker \& R. M. Rosser (Eds.), Quality of life assessment: Key issues in the 1990s (pp. 65 - 94). London: Kluwer Academic Publishers.

Kaplow, L., \& Shavell, S. (2002). Fairness versus Welfare. Cambridge, MA: Harvard University Press.

Karjalainen, K., Malmivaara, A., Mutanen, P., Roine, R., Hurri, H., \& Pohjolainen, T. (2004). Mini-intervention for subacute low back pain: two-year follow-up and modifiers of effectiveness. Spine, 29(10), 1069-1076.

Karjalainen, K., Malmivaara, A., van Tulder, M., Roine, R., Jauhiainen, M., Hurri, H., et al. (2001). Multidisciplinary biopsychosocial rehabilitation for subacute low back pain in working-age adults: a systematic review within the framework of the Cochrane Collaboration Back Review Group. Spine, 26(3), 262-269. 
Kerns, R. D., Otis, J., Rosenberg, R., \& Reid, M. C. (2003). Veterans' reports of pain and associations with ratings of health, health-risk behaviors, affective distress, and use of the healthcare system. J Rehabil Res Dev, 40(5), 371-379.

Kight, M., Gatchel, R. J., \& Wesley, L. (1999). Temporomandibular disorders: evidence for significant overlap with psychopathology. Health Psychol, 18(2), 177-182.

Kind, P. (1996). The EuroQol instrument: An index of health-related quality of life. In B. Spilker (Ed.), Quality of life and pharmacoeconomics in clinical trials (2nd ed.). Philadelphia: Lippincott-Raven.

Klarman, H., Francis, J., \& Rosenthal, G. (1968). Cost-effectiveness analysis applied to the treatment of chronic renal disease. Medical Care, 6(1), 48 - 54.

Krause, N., \& Ragland, D. R. (1994). Occupational disability due to low back pain: A new interdisciplinary classification used in a phase model of disability. Spine, 19, 1011-1020.

Krueger, A. B., \& Stone, A. A. (2008). Assessment of pain: a community-based diary survey in the USA. Lancet, 371(9623), 1519-1525.

Lawrence, W. F., \& Fleishman, J. A. (2004). Predicting EuroQoL EQ-5D preference scores from the SF-12 Health Survey in a nationally representative sample. Med Decis Making, 24(2), 160-169.

Leeman, G., Polatin, P., Gatchel, R., \& Kishino, N. (2000). Managing secondary gain in patients with pain-associated disability: A clinical perspective. Journal of Workers Compensation, 9, 25-44. 
Li, E. J., Li-Tsang, C. W., Lam, C. S., Hui, K. Y., \& Chan, C. C. (2006). The effect of a "training on work readiness" program for workers with musculoskeletal injuries: A randomized control trial (RCT) study. J Occup Rehabil.

Lindstrom, I., Ohlund, C., Eek, C., Wallin, L., Peterson, L. E., Fordyce, W. E., et al. (1992). The effect of graded activity on patients with subacute low back pain: a randomized prospective clinical study with an operant-conditioning behavioral approach. Phys Ther, 72(4), 279-290; discussion 291-273.

Loeser, J. D. (1982). Concepts of Pain. In J. Stanton-Hicks \& R. Boaz (Eds.), Chronic Low Back Pain. New York: Raven Press.

Loisel, P., Lemaire, J., Poitras, S., Durand, M. J., Champagne, F., Stock, S., et al. (2002). Cost-benefit and cost-effectiveness analysis of a disability prevention model for back pain management: a six year follow up study. Occup Environ Med, 59(12), 807-815.

Luce, B. R., Manning, W. G., Siegel, J. E., \& Lipscomb, J. (1996). Estimating costs in cost-effectiveness analysis. In M. R. Gold, J. E. Siegel, L. B. Russell \& M. C. Weinstein (Eds.), Cost-Effectiveness in Health and Medicine (pp. 176-213). New York: Oxford University Press.

Luo, X., Pietrobon, R., Sun, S. X., Liu, G. G., \& Hey, L. (2004). Estimates and patterns of direct health care expenditures among individuals with back pain in the United States. Spine, 29(1), 79-86. 
Machlin, S. R., \& Carper, K. (2007). Medical Expenditure Panel Survey Statistical Brief \#166: Expenses for office-based physician visits by specialty, 2004: Agency for Healthcare Research and Quality.

Maghout-Juratli, S., Franklin, G. M., Mirza, S. K., Wickizer, T. M., \& Fulton-Kehoe, D. (2006). Lumbar fusion outcomes in Washington State workers' compensation. Spine, 31(23), 2715-2723.

Manning, W. G., Fryback, D. G., \& Weinstein, M. C. (1996). Reflecting uncertainty in cost-effectiveness analysis. In M. R. Gold, J. E. Siegel, L. B. Russell \& M. C. Weinstein (Eds.), Cost-Effectiveness in Health and Medicine (pp. 247-275). New York: Oxford University Press.

Mannion, A. F., \& Elfering, A. (2006). Predictors of surgical outcome and their assessment. Eur Spine J, 15 Suppl 1, S93-108.

Mayer, T., McMahon, M. J., Gatchel, R. J., Sparks, B., Wright, A., \& Pegues, P. (1998). Socioeconomic outcomes of combined spine surgery and functional restoration in workers' compensation spinal disorders with matched controls. Spine, 23(5), 598-605; discussion 606.

Mayer, T., Polatin, P. B., Gatchel, R. J., Fardon, D., Herring, S., Smith, C., et al. (2003). Spine rehabilitation: Secondary and tertiary nonoperative care. The Spine Journal, 3, 28-36.

Mayer, T. G., \& Gatchel, R. J. (1988). Functional Restoration for Spinal Disorders: The Sports Medicine Approach. Philadelphia: Lea \& Febiger. 
Mayer, T. G., Gatchel, R. J., \& Evans, T. H. (2001). Effect of Age on Outcomes of Tertiary Rehabilitation for Chronic Disabling Spinal Disorders. Spine, 26.

Mayer, T. G., Gatchel, R. J., Kishino, N., Keeley, J., Capra, P., Mayer, H., et al. (1985). Objective assessment of spine function following industrial injury: A prospective study with comparison group and one-year follow-up. Spine, 10, 482-493.

Mayer, T. G., Gatchel, R. J., Mayer, H., Kishino, N. D., Keeley, J., \& Mooney, V. A. (1987). A prospective two-year study of functional restoration in industrial low back injury. An objective assessment procedure [published erratum appears in JAMA 1988 Jan 8;259(2):220]. JAMA, 258(13), 1763-1767.

Mayer, T. G., Gatchel, R. J., Porter, S., \& Theodore, B. R. (2006). Postinjury Rehabilitation/Management. In W. S. Marras \& W. Karwowski (Eds.), The Occupational Ergonomics Handbook: Interventions, Controls, and Applications in Occupational Ergonomics (2 ed.). Boca Raton, FL: CRC Press.

Mayer, T. G., \& Polatin, P. B. (2000). Tertiary nonoperative interdisciplinary programs: The functional restoration variant of the outpatient chronic pain management program. In T. G. Mayer, R. J. Gatchel \& P. B. Polatin (Eds.), Occupational Musculoskeletal Disorders: Function, Outcomes \& Evidence (pp. 639-649). Philadelphia: Lippincott, Williams \& Wilkins.

Mayer, T. G., Prescott, M., \& Gatchel, R. J. (2000). Objective outcomes evaluation: Methods and evidence. In T. G. Mayer, P. B. Polatin \& R. J. Gatchel (Eds.), 
Occupational Musculoskeletal Disorders: Function, Outcomes and Evidence (pp. 651-667). Philadelphia: Lippincott Williams \& Wilkins.

Mayer, T. G., \& Press, J. M. (2005). Musculoskeletal rehabilitation. In A. Vaccaro (Ed.), Orthopedic Knowledge Update (Vol. 8, pp. 655-661). Chicago, IL: AAOS Press.

Mayer, T. G., Towns, B. L., Neblett, R., Theodore, B. R., \& Gatchel, R. J. (2008). Chronic widespread pain in patients with occupational spinal disorders: prevalence, psychiatric comorbidity, and association with outcomes. Spine, 33(17), 1889-1897.

McGeary, D. D., Mayer, T. G., \& Gatchel, R. J. (2006). High pain ratings predict treatment failure in chronic occupational musculoskeletal disorders. Journal of Bone and Joint Surgery, 88(2), 317-325.

McGeary, D. D., Mayer, T. G., Gatchel, R. J., Anagnostis, C., \& Proctor, T. J. (2003). Gender-related differences in treatment outcomes for patients with musculoskeletal disorders. The Spine Journal, 3, 197-203.

Melzack, R., \& Casey, K. L. (1968). Sensory, motivational, and central control determinants of pain: A new conceptual model. In D. R. Kenshalo (Ed.), The Skin Senses. (pp. 423-439). Springfield, IL: Thomas.

Melzack, R., \& Wall, P. D. (1965). Pain mechanisms: A new theory. Science, 50, 971979.

Messonnier, M., \& Meltzer, M. (2003). Cost-Benefit Analysis. In A. C. Haddix, S. M. Teutsch \& P. S. Corso (Eds.), Prevention effectiveness: A guide to decision 
analysis and economic evaluation (2nd ed.). New York: Oxford University Press.

Mihaylova, B., Briggs, A., Armitage, J., Parish, S., Gray, A., \& Collins, R. (2005). Cost-effectiveness of simvastatin in people at different levels of vascular disease risk: economic analysis of a randomised trial in 20,536 individuals. Lancet, 365(9473), 1779-1785.

Patrick, D. L., Starks, H. E., Cain, K. C., Uhlmann, R. F., \& Pearlman, R. A. (1994). Measuring preferences for health states worse than death. Med Decis Making, $14,9-18$.

Patrick, L., Altmaier, E., \& Found, E. (2004). Long-term outcomes in multidisciplinary treatment of chronic low back pain: Results of a 13-year follow-up. Spine, 29, 850-855.

Pickard, A. S., Wang, Z., Walton, S. M., \& Lee, T. A. (2005). Are decisions using costutility analyses robust to choice of SF-36/SF-12 preference-based algorithm? Health Qual Life Outcomes, 3, 11.

Proctor, T., Mayer, T., Gatchel, R., \& McGeary, D. D. (2004). Unremitting health care utilization outcomes of tertiary rehabilitation of chronic musculoskeletal disorders. Journal of Bone and Joint Surgery, 86, 62-69.

Proctor, T. J., Mayer, T. G., Theodore, B., \& Gatchel, R. J. (2005). Failure to complete a functional restoration program for chronic musculoskeletal disorders: a prospective 1-year outcome study. Archives of Physical Medicine and Rehabilitation, 86(8), 1509-1515. 
Rainville, J., Kim, R. S., \& Katz, J. N. (2007). A review of 1985 Volvo Award winner in clinical science: objective assessment of spine function following industrial injury: a prospective study with comparison group and 1-year follow-up. Spine, 32(18), 2031-2034.

Rice, D., Hodgson, T., \& Epstein, A. (1985). The economic costs of illness: A replication and update. Health Care Financing Review, 7, 61-80.

Richardson, J. (1994). Cost utility analysis: What should be measured? Social Science \& Medicine, 39, 7 - 21.

Richardson, J., \& McKie, J. (2005). Empiricism, ethics and orthodox economic theory: what is the appropriate basis for decision-making in the health sector? Soc Sci Med, 60(2), 265-275.

Rivero-Arias, O., Campbell, H., Gray, A., Fairbank, J., Frost, H., \& WilsonMacDonald, J. (2005). Surgical stabilisation of the spine compared with a programme of intensive rehabilitation for the management of patients with chronic low back pain: Cost utility analysis based on a randomised controlled trial. British Medical Journal, 330(7502), 1239.

Rivero-Arias, O., Gray, A., Frost, H., Lamb, S. E., \& Stewart-Brown, S. (2006). Costutility analysis of physiotherapy treatment compared with physiotherapy advice in low back pain. Spine, 31(12), 1381-1387.

Rothman, K. J., Greenland, S., \& Lash, T. L. (2008). Modern Epidemiology (3rd ed.). Philadelphia, PA: Lippincott Williams \& Wilkins. 
Sander, R. A., \& Meyers, J. E. (1986). The relationship of disability to compensation status in railroad workers. Spine, 11(2), 141-143.

Sen, A. (1997a). Maximization and the act of choice. Econometrica, 65(4), 745 - 779.

Sen, A. (1997b). On economic inequality. Oxford: The Clarendon Press.

Skouen, J. S., Grasdal, A. L., Haldorsen, E. M., \& Ursin, H. (2002). Relative costeffectiveness of extensive and light multidisciplinary treatment programs versus treatment as usual for patients with chronic low back pain on long-term sick leave: randomized controlled study. Spine, 27(9), 901-909; discussion 909-910. Social Security Administration. (2008). How Workers' Compensation And Other Disability Payments May Affect Your Benefits (SSA Publication No. 05-10018). Retrieved. from http://www.ssa.gov/pubs/10018.html.

Sogaard, R., Bunger, C. E., Laurberg, I., \& Christensen, F. B. (2008). Costeffectiveness evaluation of an RCT in rehabilitation after lumbar spinal fusion: a low-cost, behavioural approach is cost-effective over individual exercise therapy. Eur Spine J, 17(2), 262-271.

Solberg, T. K., Olsen, J. A., Ingebrigtsen, T., Hofoss, D., \& Nygaard, O. P. (2005). Health-related quality of life assessment by the EuroQol-5D can provide costutility data in the field of low-back surgery. Eur Spine J, 14(10), 1000-1007.

Stadler, M., Schlander, M., Braeckman, M., Nguyen, T., \& Boogaerts, J. G. (2004). A cost-utility and cost-effectiveness analysis of an acute pain service. J Clin Anesth, 16(3), 159-167. 
Stewart, W. F., Ricci, J. A., Chee, E., Morganstein, D., \& Lipton, R. (2003). Lost productive time and cost due to common pain conditions in the U.S. workforce. Journal of the American Medical Association, 290(18), 2443-2454.

Stowell, A. W., Gatchel, R. J., \& Wildenstein, L. (2007). Cost-effectiveness of treatments for temporomandibular disorders: biopsychosocial intervention versus treatment as usual. J Am Dent Assoc, 138(2), 202-208.

Tabachnick, B. G., \& Fidell, L. S. (2001). Using multivariate statistics (4 ed.). Boston: Allyn \& Bacon.

Taylor, V. M., Deyo, R. A., Ciol, M., \& Kreuter, W. (1996). Surgical treatment of patients with back problems covered by workers compensation versus those with other sources of payment. Spine, 21(19), 2255-2259.

Theodore, B. R. (2007). Do Spinal Fusions Necessarily Result In Poorer Therapeutic Outcomes? , University of Texas at Arlington, Arlington, TX.

Theodore, B. R., Chan, C. C. H., \& Gatchel, R. J. (2008). Where does pain fit within healthcare delivery systems and organizations? In H. Breivik, W. I. Campbell \& M. K. Nicholas (Eds.), Clinical pain management: Practice and procedures (2nd ed.). London: Hodder Arnold.

Torrance, G. W. (1976). Social preference for health states: An empircal evaluation of three measurement techniques. Socio-Economic Planning Sciences, 10, 129 136.

Torrance, G. W. (1986). Measurement of health state utilities for economic appraisal. Journal of Health Economics, 5, 1 - 30. 
Torrance, G. W., Thomas, W. H., \& Sacket, D. L. (1972). A utility maximization model for evaluation of health care programs. Health Services Research, 7, 118 - 133.

Turk, D. C. (2002). Clinical effectiveness and cost-effectiveness of treatments for patients with chronic pain. The Clinical Journal of Pain, 18(6), 355-365.

Turk, D. C., Loeser, J. D., \& Monarch, E. S. (2002). Chronic pain: Purposes and costs of interdisciplinary pain rehabilitation programs. Trends in Evidence-Based Neuropsychiatry, 4(2), 64-69.

Turk, D. C., \& Monarch, E. S. (2002). Biopsychosocial perspective on chronic pain. In D. C. Turk \& R. J. Gatchel (Eds.), Psychological Approaches to Pain Management: A Practitioner's Handbook (2nd ed.). New York: Guilford.

Turk, D. C., \& Okifuji, A. (1997). Multidisciplinary pain centers: Boons or boondoggles? Journal of Workers' Compensation, 6, 9-26.

Turk, D. C., Swanson, K. S., \& Gatchel, R. J. (2008). Predicting opioid misuse by chronic pain patients: a systematic review and literature synthesis. Clin J Pain, 24(6), 497-508.

Turner, J. A., Franklin, G., Fulton-Kehoe, D., Sheppard, L., Stover, B., Wu, R., et al. (2008). ISSLS prize winner: Early predictors of chronic work disability: a prospective, population-based study of workers with back injuries. Spine, 33(25), 2809-2818.

U. S. General Accounting Office. (2001). SSA Disability: Other programs may provide lessons for improving return-to-work efforts: GAO-01-153. 
U.S. Bureau of Labor Statistics. (2007). Case and Demographic Characteristics for Work-related Injuries and Illnesses Involving Days Away From Work. Retrieved January 8, 2009, from http://www.bls.gov/iif/oshwc/osh/case/ostb1961.pdf

U.S. Department of Labor. (1999). Injury Compensation for Federal Employees:

Publication CA-810. Retrieved December 15, 2008, from http://www.dol.gov/esa/owcp/dfec/regs/compliance/agencyhb.pdf van der Roer, N., Boos, N., \& van Tulder, M. W. (2006). Economic evaluations: a new avenue of outcome assessment in spinal disorders. Eur Spine J, 15 Suppl 1, S109-117.

van Oostrom, S. H., Anema, J. R., Terluin, B., de Vet, H. C., Knol, D. L., \& van Mechelen, W. (2008). Cost-effectiveness of a workplace intervention for sicklisted employees with common mental disorders: design of a randomized controlled trial. BMC Public Health, 8, 12.

Vetter, T. R. (2007). The application of economic evaluation methods in the chronic pain medicine literature. Anesth Analg, 105(1), 114-118.

Von Korff, M. (1999). Pain management in primary care: An individualized steppedcare approach. In R. J. Gatchel \& D. C. Turk (Eds.), Psychosocial Factors in Pain: Critical Perspectives. New York: Guilford.

von Neumann, J., \& Morgenstern, O. (1944). Theory of Games and Economic Behavior. Princeton, NJ: Princeton University Press. 
Waddell, G., Kummel, E. G., Lotto, W. N., Graham, J. D., Hall, H., \& McCulloch, J. A. (1979). Failed lumbar disc surgery and repeat surgery following industrial injuries. J Bone Joint Surg Am, 61(2), 201-207.

Waddell, G., \& Main, C. J. (1984). Assessment of severity in low back disorders. Spine, 9, 204-208.

Ware, J. E., Kosinski, M., \& Keller, S. D. (1996). A 12-Item Short-Form Health Survey: Construction of scales and preliminary tests of reliability and validity. Medical Care, 34(3), 220-233.

Ware, J. E., Snow, K. K., Kosinski, M., \& Gandek, B. (1993). SF-36 Health Survey: Manual and Interpretation Guide. Boston: The Health Institute, New England Medical Center.

Wilson, H. D. (2007). Minimum Clinical Important Differences Of Health Outcomes In A Chronic Pain Population: Are They Predictive Of Poor Outcomes? Unpublished Dissertation, University of Texas at Arlington, Arlington.

Workers' Compensation Research Institute. (2007). Average Benefit Payment per Claim with More Than 7 Days of Lost Time, 2006/2007 and 2004/2007. Retrieved January 5, 2009, from http://www.wcrinet.org/benchmarks/benchmarks_08/benchmarks_08_fig_1.a.ht $\mathrm{ml}$

Wright, A., Mayer, T. G., \& Gatchel, R. J. (1999). Outcomes of disabling cervical spine disorders in compensation injuries. A prospective comparison to tertiary 
rehabilitation response for chronic lumbar spinal disorders. Spine, 24(2), 178183.

Yabroff, K. R., \& Mandelblatt, J. (2003). Measuring Effectiveness. In A. C. Haddix, S. M. Teutsch \& P. S. Corso (Eds.), Prevention effectiveness: A guide to decision analysis and economic evaluation (2nd ed., pp. 28-52). New York: Oxford University Press. 


\section{BIOGRAPHICAL INFORMATION}

Brian Rohan Theodore obtained the B.S. in Psychology from the University of Texas at Arlington, in Spring 2003. This was followed by an M.S. in Psychology, awarded in Summer 2007, from the University of Texas at Arlington. Following the award of the Ph.D. in Experimental Psychology based on this project, Brian will be pursuing a post-doctoral research position as a Senior Fellow with the Department of Anesthesiology and Pain Medicine at the University of Washington. Brian's research interests include the application of the biopsychosocial model for studying chronic pain, and the utilization of complex multivariate statistical models in the evaluation of risk factors associated with chronic pain and its treatment outcomes. 